\title{
Apical stress fibers enable a scaling between cell mechanical response and area in epithelial
}

tissue

Jesús M. López-Gay ${ }^{1,2}$, Hayden Nunley ${ }^{3}$, Meryl Spencer ${ }^{4}$, Florencia di Pietro ${ }^{1,2}$, Boris Guirao ${ }^{1,2}$, Floris Bosveld ${ }^{1,2}$, Olga Markova ${ }^{1,2}$, Isabelle Gaugue ${ }^{1,2}$, Stéphane Pelletier ${ }^{1,2}$, David K. Lubensky ${ }^{3,4, *}$ and Yohanns Bellaïche ${ }^{1,2,{ }^{*}}$

1 : Institut Curie, PSL Research University, CNRS UMR 3215, INSERM U934, F-75248 Paris Cedex 05, France.

2 : Sorbonne Universités, UPMC Univ Paris 06, CNRS, CNRS UMR 3215, INSERM U934, F-75005, France.

3 : Biophysics Program, University of Michigan, Ann Arbor, MI 48109-1055, USA.

4 : Department of Physics, University of Michigan, Ann Arbor, MI 48109-1040, USA.

*: Corresponding authors: dkluben@umich.edu; yohanns.bellaiche@curie.fr

One Sentence Summary: An interplay between stress fibers and tricellular junctions drives areadependent cell mechanical response. 


\begin{abstract}
Biological systems tailor their properties and behavior to their size throughout development and physiology. However, such size scaling remains poorly understood as it applies to cell mechanics and mechanosensing. By examining how the Drosophila pupal dorsal thorax epithelium responds to morphogenetic forces, we found that the number of apical stress fibers (aSFs) anchored to adherens junctions scales with cell apical area to limit larger cell elongation under mechanical stress. aSFs cluster Hippo pathway components, thereby scaling Hippo signalling and proliferation with area. This scaling is promoted by tricellular junctions mediating an increase in aSF nucleation rate and lifetime in larger cells. Development, homeostasis, and repair entail epithelial cell size changes driven by mechanical forces; our work highlights how, in turn, mechanosensitivity scales with cell size.
\end{abstract}


Within epithelial tissues, cells generate mechanical forces, which are sensed by neighboring cells (1). Numerous studies have explored the critical roles of cell mechanosensitivity in epithelial proliferation and self-organization (1). In epithelia, mechanical forces are sensed at the level of the adherens junctions (AJs), in particular by the Hippo/Yap pathway (1-3). Cell geometry, including apical cell area, varies considerably among cells within a tissue. Yet, little attention has been given to whether and how epithelial cells adapt their mechanical response to their geometry or whether such adaptation is important for tissue dynamics and morphogenesis.

\section{Apical stress fibers form in response to morphogenetic forces}

To investigate the possible interplay between cell geometry and cell mechanical response, we first aimed to better understand how epithelial tissues respond to endogenous morphogenetic forces. To this end, we used the Drosophila pupal dorsal thorax's monolayered epithelium (notum), a well-established model to study cytoskeleton dynamics, mitosis, morphogenesis and mechanics $(4-9)$.

In its posterior and central region (black dashed box in Fig. 1A, and Fig. S1A-A"), this tissue proliferates and elongates as tensile mechanical stress increases along the medial-lateral (m1) axis and becomes anisotropic between 18 and 26 hours After Pupae Formation (hAPF, Fig. 1B, Fig. S1B,C and Movie S1 $(4,10)$ ). In this region and prior to $18 \mathrm{hAPF}$, Myosin II (MyoII) is localized at the junctional cortex and in an apical medial pool (Fig. 1C, left). Between 18 and $26 \mathrm{hAPF}$, the MyoII apical-medial pool reorganizes into MyoII fibers that form at the cell apex (Fig. 1B,C and Movie S2). These fibers are oriented along the $\mathrm{m}-\mathrm{l}$ axis and are under tensile stress (Fig. 1D-F, Fig. S1D and Movie S3). The fiber tips are positioned at the level of the adherens junctions (AJs) (Fig. 1C and Fig. S1E-F"'). Known components of stress fibers (SFs), including Zyxin (Zyx), Enabled (Ena) and $\alpha$ Actinin (Actn), are distributed along or at the tips of the fibers (Fig. 1G, Fig. S1G-Q and Table S1). Since we found that MyolI fibers are apical, connected to apical AJs, and tensile, we refer to them as apical stress fibers (aSFs). aSFs oriented along the main axis of tensile stress are present in other tissue regions (green dashed box in Fig. S1 A',R,S), and Myosin-rich fibers anchored at the level of AJs have been observed in several tissues and cells types (11-14). To test whether aSF formation is a response to mechanical stress, we reduced mechanical stress in the central tissue domain by complementary approaches: by expressing a dominant negative form of MyoII heavy chain $\left(z i p^{D N}\right)$ in the lateral tissue region (Fig. S2A,A') or by applying a $20 \%$ compressive mechanical strain in the tissue plane on the living animal (Fig. 
S2B-C'). Both approaches led to a reduced number of aSFs and a disturbance of their m-1 orientation (Fig. 1H-N). Conversely, locally increasing contractility by reducing MyoII phosphatase activity leads to an increase of the number of aSFs in the surrounding cells (Fig. S2DF). We conclude that an increase in mechanical stress promotes the formation of tensile aSFs along the tissue's main stress axis.

\section{Apical stress fiber number scales with cell area to limit cell elongation}

The role of SFs is far from understood in vivo, and the relative contributions of cell-cell junctions and SFs in epithelial tissue mechanics are unexplored. We therefore investigated the roles of aSFs in morphogenesis by both experimental and modeling approaches. Since aSFs are under tension (Fig. 1F, Fig. S3K and Movie S3 and S4), we tested whether aSFs regulate cell and tissue elongation. Towards this goal, we screened for loss of function mutants affecting aSF number at 26 hAPF (Table S1). Abrogating Actn function (using either RNAi or a CRISPR/Cas9-generated null allele) in the central posterior region of the tissue leads to a drastic decrease in aSF number per cell as well as to an increase in cell and tissue elongation along the main axis of mechanical stress (Fig. 2A-D and Fig. S3A-H'). This increase in elongation occurred without a major change in the tissue's global mechanical stress (Fig. S3I); this indicates that aSFs prevent cell and tissue elongation in response to mechanical stress generated during morphogenesis. To explore the respective mechanical roles of aSFs and AJs, we then used laser ablation to estimate the tensions of aSFs and AJs (Fig. S1D, Fig. S3J-K). The recoil velocity upon aSF ablation was around onethird of that observed for ablation of AJs aligned with the m-l axis; this suggests that aSFs have lower tension than similarly oriented AJs (Fig. 2E, Fig. S3K and Movie S3 and S4). By sequentially ablating an aSF and then an AJ from the same cell, we found that aSFs diminish the tension exerted on the AJ (Fig. 2E, Movie S5 and S6). Consistently, AJ recoil velocity upon laser ablation was increased in $a c t n^{R N A i}$ cells relative to control cells at $26 \mathrm{hAPF}$, but not at $18 \mathrm{hAPF}$ when aSFs are absent (Fig. 2F). To further understand aSFs' contribution to cell and tissue morphogenesis, we considered a simple vertex model of a regular cell packing in mechanical equilibrium with or without aSFs (Fig. 2G,H, Supplementary Text and Fig. S4). As observed experimentally, the model shows that aSFs oriented along the main stress axis limit cell and tissue elongation (Fig. 2H and Fig. S4). Furthermore, mirroring our observation in $a c t n^{R N A i}$ cells, the model shows that, at high stress anisotropies, aSFs are more efficient at limiting cell elongation than simply upregulating tension along AJs (Fig. 2H and Fig. S4). The model moreover predicts 
that cells with a larger apical area require more aSFs (or more tension per aSF) to limit cell elongation to maintain the same aspect ratio under anisotropic mechanical stress (Fig. 2I, Supplementary Text and Fig. S4).

Based on these predictions, we investigated the relationship between individual aSF tension, aSF number per cell and cell apical area at 26 hAPF. First, laser ablation suggests that the tension supported by one aSF is independent of cell apical area (Fig. S3L). Second, the number of aSFs per cell scales with the cell's apical area (Fig. 2J and Fig. S3M,N). Finally, when we ablated more than one aSF within a given cell, the cell elongated more with each additional aSF ablation (Fig. 2K, Movie S7). We then investigated whether a change in cell apical area leads to a change in the number of aSFs. By comparing the cell area and aSF number per cell in interphase cells one hour before and one hour after cytokinesis, we found that a reduced cell apical area is associated with a reduced number of aSFs per cell (Fig. S3O). Conversely, preventing cell division or cytokinesis leads to an increase in cell area and an increase in aSF number per cell (Fig. 2L and Fig. S3O).

We then tested whether the scaling between cell area and aSF number is important for limiting cell elongation. Fractional cell elongation is independent of cell area in control cells (Fig. $\mathrm{S} 3 \mathrm{P}$ ), and aSF ablation leads to a cell elongation that increases with cell area (Fig. S3P,Q). Accordingly, the elongation of $a c t n^{R N A i}$ cells increases with their apical area as the stress anisotropy increases (Fig. 2M and Fig. S3P). We conclude that a scaling between aSF number and cell area reduces tissue elongation under anisotropic stress by ensuring that cell elongation is independent of cell area.

\section{Scaling between cell area and Hippo/Yki signaling via aSF number scaling.}

We next explored whether the scaling between cell area and aSF number translates into biochemical regulation in conditions of mechanical stress. aSFs are located away from the nucleus (Fig. S1E'); this suggests that aSFs do not regulate Yap/Taz (Yorkie, Yki in Drosophila) activity by direct nuclear deformation, as found in individual cultured cells $(2,15)$. We therefore investigated alternative mechanisms through which aSFs might modulate Hippo/Yki activity in epithelial tissues. The LIM domain protein Ajuba (Jub) is a component of the Hippo/Yki pathway that binds to the Warts (Wts) kinase. Jub binding results in Wts inhibition and thus Yki activity upregulation $(16,17)$. Previous findings indicate that an increase of MyoII contractility leads to the increased recruitment of Jub to the AJs and that this recruitment promotes Hippo/Yki signaling 
(18-21). In the dorsal thorax, we uncovered that at high mechanical stress (26 hAPF), Jub and Wts form clusters at the tips of the aSFs (Fig. 3A,B and Fig. S5A-D). Furthermore, quantitative analyses of the Jub and Wts distributions showed that the number of Jub and Wts clusters as well as the ratio of Jub and Wts intensity in clusters to that elsewhere along the $\mathrm{AJ}$ (ratio ${ }^{\text {in/out }}$ of cluster) increases between low stress (18 hAPF) and high stress (26 hAPF) (Fig. 3C and Fig. S5E-F). Accordingly, Jub and Wts colocalization increases under high mechanical stress (Fig. 3D-D' and Fig. S5G). Thus, an increase in mechanical stress correlates with the formation of Jub and Wts coclusters at the tips of the aSFs along the AJs. To determine whether Jub clustering modulates Hippo signaling, we used the Cry2Olig optogenetic clustering system (22) to induce Jub clustering independent of aSF formation. We found that light-induced clustering of Jub is sufficient to cocluster Wts and to upregulate Yki activity (Fig. S5H-J, Movie S8). Next, we explored whether the formation of aSFs and their associated tension modulate Jub and Wts clustering, Hippo/Yki activity, and cell proliferation. High-resolution time-lapse imaging showed that as an aSF forms or is displaced along the AJ, cortical Jub:mKate2 and Wts:CitFP flow and accumulate at the aSF tip (Fig. 3E, Fig. S5K,K' and Movie S9). Conversely, upon ablation of a previously formed aSF, the Jub:GFP or Wts:CitFP clusters initially present at that aSF's tip are strongly reduced (Fig. 3F-G, Fig. S5L-M and Movie S10). While the loss of Actn function does not affect the total amount of Jub and Wts at the junction (Fig. S5P,Q), it decreases the number of Jub and Wts clusters and their colocalization as well as the ratios ${ }^{\text {in/out }}$ of Jub and Wts clusters (Fig. S5R-S and Fig. 3H). Last, we found that the loss of Actn function leads to a decrease of ban-nls:GFP Yki transcriptional reporter expression (23) and cell proliferation, specifically under high stress (Fig. 3I,J and Fig. S5T-V). We conclude that aSFs promote the co-clustering of Jub and Wts at their tips, accounting for the downregulation of Wts activity and the upregulation of Yki transcriptional activity.

Having characterized the link between aSF formation and Yki transcriptional activation at $26 \mathrm{hAPF}$, we explored whether the scaling between aSF number and cell apical area might result in the scaling of Yki activity with cell apical area. We quantified the intensity of ban-nls:GFP as a function of cell apical area. Ban-nls:GFP levels increase as cell apical area increases from 10 to $32 \mu \mathrm{m}^{2}$ and then reach a plateau for cells between 32 and $40 \mu \mathrm{m}^{2}$ (Fig. 4A and Fig. S6A). In contrast, the level of $n l s: G F P$ under the control of a ubiquitin promoter (Ubi-nls:GFP) is independent of cell apical area (Fig. 4A). In agreement with the proposed role of Jub clustering in Yki activity regulation, we found that the ban-nls:GFP signal increases with the Jub ratio ${ }^{\text {in/out }}$ (Fig. 
4B). Furthermore, we observed that under stress both the Jub and Wts ratios ${ }^{\text {in/out }}$ of clusters increase with cell apical area, consistent with the notion that a larger fraction of Wts is inhibited in larger cells under stress (Fig. 4C and Fig. S6B,C). If aSFs contribute to the scaling between cell size and Hippo/Yki pathway activation, the impact of Actn loss of function on Wts and Jub clustering should be more pronounced in larger cells. Accordingly, Jub and Wts clustering is not affected in small $a c t n^{R N A i}$ cells, whereas clear differences exist between large $a c t n^{R N A i}$ cells and large control cells (Fig. 4D,E and Fig. S6D). Furthermore, we observed that the effect of Actn loss of function on cell proliferation mirrors the defects observed in Jub and Wts clustering in $\operatorname{actn}^{R N A i}$ cells (Fig. 4F). Finally, we increased cell apical area by preventing cytokinesis and observed a corresponding increase in both Jub ratio ${ }^{\text {in/out }}$ of clusters and ban-nlsGFP level (Fig. S6E,F). Together, these findings indicate that the scaling between cell area and aSF number leads to a scaling between cell area and the clustering of Jub and Wts, and thus to the scaling of Hippo/Yki signaling activity with apical cell size in epithelial tissues.

\section{Tricellular junctions promote scaling between aSF number and apical area.}

Having established the role of aSFs in scaling cells' mechanical and biochemical responses under mechanical stress, we next explored the origins of the scaling of aSF number with cell apical area. SF formation is best studied in individual cells where the spatial organization of the SFs depends on cell shape $(24,25)$. Yet, even in this context, the mechanisms controlling SF number and dynamics are not fully understood $(24,25)$. To investigate how the scaling between cell size and aSF number is achieved in epithelial tissues under anisotropic stress, we analyzed aSF dynamics at $26 \mathrm{hAPF}$. Via live imaging of aSFs, we found that aSFs form at curved regions of cellcell junctions aligned with the main axis of tensile stress (Fig. 5A-B and Movie S11). In epithelial tissues, tricellular junctions (TCJs), points where three cells meet, are often the most curved regions of the cell apical contour (26). Accordingly, aSFs mainly nucleate at TCJs (Fig. 5A-C). After nucleation, aSFs sweep across the cell as they peel from the cortex, most often breaking as they encounter another TCJ (Fig. 5A-C). A TCJ's propensity to nucleate or to break aSFs varies simply as a function of the orientation of the TCJ's bisector with respect to the main stress axis (Fig. S7AF, Fig. S8L-M,T and Supplementary Text). Thus, we could classify a TCJ as a "nucleating TCJ" or a "breaking TCJ" based on this orientation. While the exact mechanical and molecular mechanisms driving aSF nucleation and breakage at TCJs remain to be understood, the TCJs' role in both processes suggests a geometric mechanism for the scaling of aSF number with cell apical 
area based on the following trends: The larger cells in a tissue have more TCJs (27) and thus might have higher rates of aSF nucleation. A typical nucleating TCJ is also farther from a typical breaking TCJ in larger cells, so that aSFs in larger cells could be expected to take longer to travel from one to the other. Together, these two effects would lead to larger cells' having more aSFs due to an increase in both aSF nucleation rate and aSF lifetime.

To test this hypothesis, we analyzed how aSF nucleation rates and lifetimes differ between small and large cells. Large cells on average have higher aSF nucleation rates and lifetimes than small cells, in agreement with our proposed geometric mechanism (Fig. S8N-R). Next, we performed computer simulations to quantify how much of the observed scaling of aSF number with cell apical area could be explained by differences in the number of TCJs and their positions. In these simulations, we considered a simple model where only the geometric effects are present; we did not include any explicit dependence on cell size (see Supplementary Text). We applied this model to cell shapes and orientations taken directly from experimental images at $26 \mathrm{hAPF}$. We found that our geometric TCJ model explains around 75\% of the observed variation of aSF number with cell apical area with only one free parameter. This parameter can be interpreted as essentially equivalent to the junctional cortex thickness, and the fitted value agrees well with direct thickness measurements $(0.50 \pm 0.12 \mu \mathrm{m}$ versus $0.51 \pm 0.12 \mu \mathrm{m}$; Fig. 5D, Supplementary Text and Fig. S8S).

To further show that the number of aSFs within a cell depends on the properties of its TCJs, we sought to vary TCJ number and position independently of cell apical area and in a population of genetically identical cells. To accomplish this, we performed two distinct analyses. First, we took advantage of the fact that a large cell in contact with smaller cells typically has more TCJs than a large cell surrounded by other large cells (28). To generate large cells with different local environments, we induced clones of large cells by blocking the G2/M transition through overexpression of $t r b l$ ( $t r b l^{U P}$ clones) and compared $t r b l^{U P}$ cells at the boundary of the clone (border cells) to cells within the clone (bulk cells, Fig. 5E). As anticipated, $\mathrm{rrbl}^{U P}$ cells at the boundary of the clone have more nucleating TCJs (Fig. 5F) and thus might be expected to have more aSFs. To verify that no other differences in cell shape or TCJ distribution lead to a countervailing difference in predicted aSF lifetime between border and bulk cells, we simulated our geometric model of aSF nucleation and breakage on cell sizes and shapes taken from our images of $\mathrm{rrbl}^{U P}$ clones; the model indeed predicts that $t r b l^{U P}$ cells at the boundary of the clone should have more aSFs per cell than ${ }_{t r b l} l^{U P}$ bulk cells (Fig. 5I and Fig. S9A-G). Further, we found experimentally that, controlling for 
any small difference in cell size between these two populations, cells at the boundary have on average more aSFs than cells within the clone (Fig. 5G,H and Fig. S9A-G). Thus, these results substantiate our hypothesis that an increase in the number of TCJs correlates with an increase in the number of aSFs.

We then analyzed whether the spatial distribution of TCJs modulates aSF number. If the distance between the nucleating TCJ and the breaking TCJ(s) is critical to control aSF number, we predict that cells that are elongated orthogonal to the uniaxial mechanical stress (hereafter referred to as ortho-elongated) should have more aSFs than cells of a similar size elongated parallel to the main stress axis (Fig. 5J). By restricting our analysis to such ortho-elongated cells, we showed that at constant nucleating TCJ number and cell size, the number of aSFs is higher in ortho-elongated cells, as predicted (Fig. 5K-O and Fig. S9H-J). Based on quantitative analyses of aSF nucleation and lifetime, modeling and experiments, we propose that the number and positions of TCJs are major contributors to the scaling of aSF number with cell apical area in tissues under uniaxial tension (Fig. S10). TCJs thus play an unexpected role in the regulation of cells' mechanical properties and signaling under mechanical stress.

\section{Discussion}

Scaling between body size and metabolic or biomechanical properties has been the subject of intense study for decades $(29,30)$. More recently, investigations of the scaling of morphogen gradients with tissue area or length have unveiled novel mechanisms of cell fate specification during development $(31,32)$. Here, we have uncovered a scaling between the number of aSFs per cell and cell apical area in an epithelial tissue. We have furthermore provided evidence that this scaling is critical to control cell and tissue elongation as well as proliferation in response to epithelial morphogenetic forces. Existing work has emphasized the roles of SFs as anchors to extracellular matrix (ECM) via focal adhesions (FAs) and the SFs' roles in cell locomotion, cell mechanics and Hippo/YAP regulation $(24,33)$. Our work highlights the contributions of aSFs anchored to AJs as regulators of both tissue morphogenesis and proliferation under physiological morphogenetic mechanical stress. We have also found that aSFs promote the co-clustering of Jub and Wts in the control of Hippo/YAP signalling. The full characterization of the molecular interactions between aSF components and Hippo/YAP regulators at the tips of the aSFs will be critical to decipher how aSFs promote the recruitment of Jub to the aSF tips in response to tissue 
stress. Interestingly, mammalian Jub localizes at ECM-integrin FAs (34); this recruitment of Jub to FAs might provide a complementary mechanism of Hippo/YAP mechanical regulation in individual cells $(2,15,33,35-37)$. Cell cycle regulation is well predicted by a combination of cell area and tension in multicellular assemblies in culture (38). Therefore, it will be useful to analyze whether the mechanisms we uncovered might generally explain the interplay between cell area and mechanical forces in cell proliferation control. Earlier studies have shown that oriented cell divisions relax uniaxial tissue stress in a manner that depends on cell elongation (39); it will be of interest to explore how aSFs, by limiting elongation while promoting the division of larger area cells, might modulate tissue stress or mechanical properties. Our characterization of the aSF dynamics illustrates that the number and distribution of TCJs largely account for the scaling between cell apical area and aSF number. Thus, our work defines a functional link between TCJs and aSFs. As TCJ and SFs are prevalent biological structures $(24,26)$, the molecular characterization of their interplay might shed light on numerous aspects of tissue mechanics, proliferation and morphogenesis. 


\section{References and Notes:}

1. E. Hannezo, C.-P. Heisenberg, Mechanochemical Feedback Loops in Development and Disease. Cell. 178, 12-25 (2019).

2. J. R. Davis, N. Tapon, Hippo signalling during development. Dev. 146 (2019), doi:10.1242/dev.167106.

3. D. Pinheiro, Y. Bellaïche, Mechanical Force-Driven Adherens Junction Remodeling and Epithelial Dynamics. Dev. Cell. 47, 3-19 (2018).

4. F. Bosveld et al., Mechanical Control of Morphogenesis by Fat/Dachsous/Four-Jointed Planar Cell Polarity Pathway. Science (80-. ). 336, 724-727 (2012).

5. R. Levayer, C. Dupont, E. Moreno, Tissue Crowding Induces Caspase-Dependent Competition for Space. Curr. Biol. 26, 670-7 (2016).

6. S. Curran et al., Myosin II Controls Junction Fluctuations to Guide Epithelial Tissue Ordering. Dev. Cell. 43, 480-492.e6 (2017).

7. N. Founounou, N. Loyer, R. Le Borgne, Septins Regulate the Contractility of the Actomyosin Ring to Enable Adherens Junction Remodeling during Cytokinesis of Epithelial Cells. Dev. Cell. 24, 242-255 (2013).

8. F. Bosveld et al., Epithelial tricellular junctions act as interphase cell shape sensors to orient mitosis. Nature. 530, 495-498 (2016).

9. D. Pinheiro et al., Transmission of cytokinesis forces via E-cadherin dilution and actomyosin flows. Nature. 545, 103-107 (2017).

10. B. Guirao et al., Unified quantitative characterization of epithelial tissue development. Elife. 4, e08519 (2015).

11. V. Vasioukhin, C. Bauer, M. Yin, E. Fuchs, Directed actin polymerization is the driving force for epithelial cell- cell adhesion. Cell. 100, 209-219 (2000).

12. J. Millán et al., Adherens junctions connect stress fibres between adjacent endothelial cells. BMC Biol. 8 (2010), doi:10.1186/1741-7007-8-11.

13. M. K. L. Han, J. de Rooij, Converging and Unique Mechanisms of Mechanotransduction at Adhesion Sites. Trends Cell Biol. 26, 612-623 (2016).

14. S. Huveneers et al., Vinculin associates with endothelial VE-cadherin junctions to control force-dependent remodeling. J. Cell Biol. 196, 641-652 (2012).

15. A. Elosegui-Artola et al., Force Triggers YAP Nuclear Entry by Regulating Transport across Nuclear Pores. Cell. 171, 1397-1410.e14 (2017).

16. M. Das Thakur et al., Ajuba LIM Proteins Are Negative Regulators of the Hippo Signaling Pathway. Curr. Biol. 20, 657-662 (2010).

17. Y. Abe, M. Ohsugi, K. Haraguchi, J. Fujimoto, T. Yamamoto, LATS2-Ajuba complex regulates $\gamma$-tubulin recruitment to centrosomes and spindle organization during mitosis. FEBS Lett. 580, 782-788 (2006).

18. C. Rauskolb, S. Sun, G. Sun, Y. Pan, K. D. Irvine, Cytoskeletal tension inhibits Hippo signaling through an Ajuba-Warts complex. Cell. 158, 143-156 (2014).

19. H. Alégot et al., Recruitment of Jub by $\alpha$-catenin promotes Yki activity and Drosophila wing growth. J. Cell Sci. 132 (2019), doi:10.1242/jcs.222018. 
20. C. Ibar et al., Tension-dependent regulation of mammalian Hippo signaling through LIMD1. J. Cell Sci. 131, 1-16 (2018).

21. R. Sarpal et al., Role of $\alpha$-Catenin and its mechanosensing properties in regulating Hippo/YAP-dependent tissue growth. PLoS Genet. 15, e1008454 (2019).

22. A. Taslimi et al., An optimized optogenetic clustering tool for probing protein interaction and function. Nat. Commun. 5, 4925 (2014).

23. H. Matakatsu, S. S. Blair, Separating planar cell polarity and Hippo pathway activities of the protocadherins Fat and Dachsous. Development. 139, 1498-508 (2012).

24. L. Blanchoin, R. Boujemaa-Paterski, C. Sykes, J. Plastino, Actin Dynamics, Architecture, and Mechanics in Cell Motility. Physiol. Rev. 94, 235-263 (2014).

25. E. Kassianidou et al., Extracellular Matrix Geometry and Initial Adhesive Position Determine Stress Fiber Network Organization during Cell Spreading. Cell Rep. 27, 1897 1909.e4 (2019).

26. T. Higashi, A. L. Miller, Tricellular junctions: how to build junctions at the TRICkiest points of epithelial cells. Mol. Biol. Cell. 28, 2023-2034 (2017).

27. M. C. Gibson, A. B. Patel, R. Nagpal, N. Perrimon, The emergence of geometric order in proliferating metazoan epithelia. Nature. 442, 1038-1041 (2006).

28. W. T. Gibson et al., Control of the mitotic cleavage plane by local epithelial topology. Cell. 144, 427-438 (2011).

29. T. A. McMahon, J. T. Bonner, On size and life (Scientific American Library, 1983).

30. A. J. Spence, Scaling in biology. Curr. Biol. 19, R57-61 (2009).

31. B.-Z. Shilo, N. Barkai, Buffering Global Variability of Morphogen Gradients. Dev. Cell. 40, 429-438 (2017).

32. D. Čapek, P. Müller, Positional information and tissue scaling during development and regeneration. Development. 146 (2019), doi:10.1242/dev.177709.

33. K. D. Irvine, B. I. Shraiman, Mechanical control of growth: ideas, facts and challenges. Development. 144, 4238-4248 (2017).

34. S. J. Pratt et al., The LIM protein Ajuba influences p130Cas localization and Rac1 activity during cell migration. J. Cell Biol. 168, 813-824 (2005).

35. M. Aragona et al., A mechanical checkpoint controls multicellular growth through YAP/TAZ regulation by actin-processing factors. Cell. 154, 1047-1059 (2013).

36. K. Wada, K. Itoga, T. Okano, S. Yonemura, H. Sasaki, Hippo pathway regulation by cell morphology and stress fibers. 3914, 3907-3914 (2011).

37. Z. Meng et al., RAP2 mediates mechanoresponses of the Hippo pathway. Nature. 560, 655-660 (2018).

38. M. Uroz et al., Regulation of cell cycle progression by cell-cell and cell-matrix forces. Nat. Cell Biol. 20, 646-654 (2018).

39. B. G. Godard, C. P. Heisenberg, Cell division and tissue mechanics. Curr. Opin. Cell Biol. 60, 114-120 (2019).

40. T. Xu, G. M. Rubin, Analysis of genetic mosaics in developing and adult Drosophila tissues. Development. 117, 1223-1237 (1993).

41. T. Lee, L. Luo, Mosaic Analysis with a Repressible Cell Marker for Studies of Gene 
Function in Neuronal Morphogenesis. Neuron. 22, 451-461 (1999).

42. A. H. Brand, N. Perrimon, Targeted gene expression as a means of altering cell fates and generating dominant phenotypes. Development. 118, 401-415 (1993).

43. S. E. McGuire, P. T. Le, A. J. Osborn, K. Matsumoto, R. L. Davis, Spatiotemporal Rescue of Memory Dysfunction in Drosophila. Science (80-. ). 302, 1765-1768 (2003).

44. S. J. Gratz et al., Highly specific and efficient CRISPR/Cas9-catalyzed homology-directed repair in Drosophila. Genetics. 196, 961-71 (2014).

45. F. Port, H.-M. Chen, T. Lee, S. L. Bullock, Optimized CRISPR/Cas tools for efficient germline and somatic genome engineering in Drosophila. Proc. Natl. Acad. Sci. U. S. A. 111, E2967-76 (2014).

46. J. Huang, W. Zhou, A. M. Watson, Y. N. Jan, Y. Hong, Efficient ends-out gene targeting in drosophila. Genetics. 180, 703-707 (2008).

47. D. Sabino, N. H. Brown, R. Basto, Drosophila Ajuba is not an Aurora-A activator but is required to maintain Aurora-A at the centrosome. J. Cell Sci. 124, 1156-1166 (2011).

48. P. L. Bardet et al., PTEN Controls Junction Lengthening and Stability during Cell Rearrangement in Epithelial Tissue. Dev. Cell. 25, 534-546 (2013).

49. R. E. Dawes-Hoang et al., folded gastrulation, cell shape change and the control of myosin localization. Development. 132, 4165-78 (2005).

50. J. M. Urbano, H. W. Naylor, E. Scarpa, L. Muresan, B. Sanson, Suppression of epithelial folding at actomyosin-enriched compartment boundaries downstream of Wingless signalling in Drosophila. Development. 145 (2018), doi:10.1242/dev.155325.

51. T. Mizuno, K. Tsutsui, Y. Nishida, Drosophila myosin phosphatase and its role in dorsal closure. Development. 129, 1215-1223 (2002).

52. A. D. Herbert, A. M. Carr, E. Hoffmann, M. Lichten, FindFoci: A focus detection algorithm with automated parameter training that closely matches human assignments, reduces human inconsistencies and increases speed of analysis. PLoS One. 9 (2014), doi:10.1371/journal.pone.0114749.

53. E. O. Lebigot, Welcome to the uncertainties package - uncertainties Python package 3.0.3 documentation, (available at https://pythonhosted.org/uncertainties/).

54. G. B. Blanchard et al., Tissue tectonics: Morphogenetic strain rates, cell shape change and intercalation. Nat. Methods. 6, 458-464 (2009).

55. L. Zhang et al., The TEAD/TEF family of transcription factor Scalloped mediates Hippo signaling in organ size control. Dev. Cell. 14, 377-87 (2008).

56. F. Pedregosa et al., "Scikit-learn: Machine Learning in Python" (Microtome Pub-lishing, 2011), (available at https://hal.inria.fr/hal-00650905v2).

57. P. Virtanen et al., SciPy 1.0--Fundamental Algorithms for Scientific Computing in Python (2019) (available at http://arxiv.org/abs/1907.10121).

58. M. P. Allen, Understanding Regression Analysis (Springer US, 1997).

59. G. Kanji, 100 Statistical Tests (SAGE Publications Ltd, 2012).

60. T. Nagai, H. Honda, A dynamic cell model for the formation of epithelial tissues. Philos. Mag. B. 81, 699-719 (2001).

61. R. Farhadifar, J. C. Roper, B. Aigouy, S. Eaton, F. Julicher, The influence of cell 
mechanics, cell-cell interactions, and proliferation on epithelial packing. Curr Biol. 17, 2095-2104 (2007).

62. A. G. Fletcher, M. Osterfield, R. E. Baker, S. Y. Shvartsman, Vertex models of epithelial morphogenesis. Biophys J. 106, 2291-2304 (2014).

63. M. Rauzi, P. Verant, T. Lecuit, P. F. Lenne, Nature and anisotropy of cortical forces orienting Drosophila tissue morphogenesis. Nat Cell Biol. 10, 1401-1410 (2008).

64. B. Aigouy et al., Cell flow reorients the axis of planar polarity in the wing epithelium of Drosophila. Cell. 142, 773-786 (2010).

65. M. Aliee et al., Physical mechanisms shaping the Drosophila dorsoventral compartment boundary. Curr Biol. 22, 967-976 (2012).

66. G. Salbreux, L. K. Barthel, P. A. Raymond, D. K. Lubensky, Coupling mechanical deformations and planar cell polarity to create regular patterns in the zebrafish retina. PLoS Comput Biol. 8, e1002618 (2012).

67. P. A. Raymond et al., Patterning the cone mosaic array in zebrafish retina requires specification of ultraviolet-sensitive cones. PLoS One. 9, e85325 (2014).

68. A. F. Mertz et al., Cadherin-based intercellular adhesions organize epithelial cell-matrix traction forces. Proc Natl Acad Sci U S A. 110, 842-847 (2013).

69. M. A. Spencer, J. Lopez-Gay, H. Nunley, Y. Bellaïche, D. K. Lubensky, Multicellular actomyosin cables in epithelia under external anisotropic stress. arXiv e-prints (2018), p. arXiv:1809.04569, (available at https://ui.adsabs.harvard.edu/abs/2018arXiv180904569S).

70. D. Bi, J. H. Lopez, J. M. Schwarz, M. L. Manning, Energy barriers and cell migration in densely packed tissues. Soft Matter. 10, 1885-1890 (2014).

71. D. Bi, J. H. Lopez, J. M. Schwarz, M. L. Manning, A density-independent rigidity transition in biological tissues. Nat. Phys. 11, 1074-1079 (2015).

72. D. Bi, X. Yang, M. C. Marchetti, M. L. Manning, Motility-driven glass and jamming transitions in biological tissues. Phys Rev X. 6 (2016), doi:10.1103/PhysRevX.6.021011.

73. D. L. Weaire, S. C. N.-Q. . W. 1999 Hutzler, The physics of foams (Clarendon Press, Oxford; New York, 1999).

74. I. Cantat et al., Foams : structure and dynamics (Oxford University Press, Oxford; New York, NY, United States of America, First Engl., 2013).

75. C. C. Reyes et al., Anillin regulates cell-cell junction integrity by organizing junctional accumulation of Rho-GTP and actomyosin. Curr Biol. 24, 1263-1270 (2014).

76. C. Collinet, M. Rauzi, P. F. Lenne, T. Lecuit, Local and tissue-scale forces drive oriented junction growth during tissue extension. Nat. Cell Biol. 17, 1247-1258 (2015).

77. K. Ikawa, K. Sugimura, AIP1 and cofilin ensure a resistance to tissue tension and promote directional cell rearrangement. Nat Commun. 9, 3295 (2018).

78. J. H. Wang, P. Goldschmidt-Clermont, F. C. Yin, Contractility affects stress fiber remodeling and reorientation of endothelial cells subjected to cyclic mechanical stretching. Ann Biomed Eng. 28, 1165-1171 (2000).

79. T. Ishizaki et al., Coordination of microtubules and the actin cytoskeleton by the Rho effector mDia1. Nat Cell Biol. 3, 8-14 (2001).

80. R. Kaunas, P. Nguyen, S. Usami, S. Chien, Cooperative effects of Rho and mechanical 
stretch on stress fiber organization. Proc Natl Acad Sci U S A. 102, 15895-15900 (2005).

81. N. Elkhatib et al., Fascin plays a role in stress fiber organization and focal adhesion disassembly. Curr. Biol. 24, 1492-9 (2014).

82. N. D. Bade, R. D. Kamien, R. K. Assoian, K. J. Stebe, Curvature and Rho activation differentially control the alignment of cells and stress fibers. Sci Adv. 3, e1700150 (2017).

83. E. Kassianidou, C. A. Brand, U. S. Schwarz, S. Kumar, Geometry and network connectivity govern the mechanics of stress fibers. Proc Natl Acad Sci U S A. 114, 2622 2627 (2017).

84. P. W. Oakes et al., Optogenetic control of RhoA reveals zyxin-mediated elasticity of stress fibres. Nat Commun. 8, 15817 (2017).

85. H. G. Yevick, P. W. Miller, J. Dunkel, A. C. Martin, Structural Redundancy in Supracellular Actomyosin Networks Enables Robust Tissue Folding. Dev. Cell. 50, 586598.e3 (2019).

86. A. Nestor-Bergmann, E. Johns, S. Woolner, O. E. Jensen, Mechanical characterization of disordered and anisotropic cellular monolayers. Phys Rev E. 97, 52409 (2018).

87. W. Choi et al., Remodeling the zonula adherens in response to tension and the role of afadin in this response. J Cell Biol. 213, 243-260 (2016).

88. N. Noll, M. Mani, I. Heemskerk, S. J. Streichan, B. I. Shraiman, Active Tension Network model suggests an exotic mechanical state realized in epithelial tissues. Nat Phys. 13, 1221-1226 (2017).

89. G. Charras, A. S. Yap, Tensile Forces and Mechanotransduction at Cell-Cell Junctions. Curr Biol. 28, R445-R457 (2018).

90. G. R. Kale et al., Distinct contributions of tensile and shear stress on E-cadherin levels during morphogenesis. Nat Commun. 9, 5021 (2018).

91. P. I. Teixeira, F. Graner, M. A. Fortes, Mixing and sorting of bidisperse two-dimensional bubbles. Eur Phys J E Soft Matter. 9, 161-169 (2002).

92. F. Brochard-Wyart, P. de Gennes, Unbinding of adhesive vesicles. Comptes Rendus Phys. 4, 281-287 (2003).

93. T. M. Finegan et al., The tricellular vertex-specific adhesion molecule Sidekick facilitates polarised cell intercalation during Drosophila axis extension. PLoS Biol. 17, 704932 (2019).

94. A. Letizia et al., Sidekick Is a Key Component of Tricellular Adherens Junctions that Acts to Resolve Cell Rearrangements. Dev. Cell. 50, 313-326.e5 (2019).

95. H. Uechi, E. Kuranaga, The Tricellular Junction Protein Sidekick Regulates Vertex Dynamics to Promote Bicellular Junction Extension. Dev. Cell. 50, 327-338.e5 (2019).

96. M. Calleja, E. Moreno, S. Pelaz, G. Morata, Visualization of Gene Expression in Living Adult Drosophila. Science (80-. ). 274, 252-255 (1996).

97. J. L. Mummery-Widmer et al., Genome-wide analysis of Notch signalling in Drosophila by transgenic RNAi. Nature. 458, 987-992 (2009).

98. N. Tamura, K. Ohno, T. Katayama, N. Kanayama, K. Sato, The PDZ-LIM protein CLP36 is required for actin stress fiber formation and focal adhesion assembly in BeWo cells. Biochem. Biophys. Res. Commun. 364, 589-594 (2007). 
99. X. Lin et al., Z-disc-associated, alternatively spliced, PDZ motif-containing protein (ZASP) mutations in the actin-binding domain cause disruption of skeletal muscle actin filaments in myofibrillar myopathy. J. Biol. Chem. 289, 13615-13626 (2014).

100. M. A. Smith et al., LIM domains target actin regulators paxillin and zyxin to sites of stress fiber strain. PLoS One. 8, e69378 (2013).

101. M. Maninová, T. Vomastek, Dorsal stress fibers, transverse actin arcs, and perinuclear actin fibers form an interconnected network that induces nuclear movement in polarizing fibroblasts. FEBS J. 283, 3676-3693 (2016).

102. S. Yamashiro-Matsumura, F. Matsumura, Intracellular localization of the $55-\mathrm{kD}$ actinbundling protein in cultured cells: spatial relationships with actin, alpha-actinin, tropomyosin, and fimbrin. J. Cell Biol. 103, 631-40 (1986).

103. W. W. Carley, A. Bretscher, W. W. Webb, F-actin aggregates in transformed cells contain alpha-actinin and fimbrin but apparently lack tropomyosin. Eur. J. Cell Biol. 39, 313-20 (1986).

104. B. Machnicka, R. Grochowalska, D. M. Bogusławska, A. F. Sikorski, The role of spectrin in cell adhesion and cell-cell contact. Exp. Biol. Med. (Maywood). 244, 1303-1312 (2019).

105. a J. Ehrlicher, F. Nakamura, J. H. Hartwig, D. a Weitz, T. P. Stossel, Mechanical strain in actin networks regulates FilGAP and integrin binding to filamin A. Nature. 478, 260-263 (2011).

106. H.-M. Chen, Y.-H. Lin, Y.-M. Cheng, L.-Y. C. Wing, S.-J. Tsai, Overexpression of integrin- $\beta 1$ in leiomyoma promotes cell spreading and proliferation. J. Clin. Endocrinol. Metab. 98, E837-46 (2013).

107. C. Albiges-Rizo, P. Frachet, M. R. Block, Down regulation of talin alters cell adhesion and the processing of the $\alpha 5 \beta 1$ integrin. J. Cell Sci. 108, 3317-3329 (1995).

108. G. Gateva, S. Tojkander, S. Koho, O. Carpen, P. Lappalainen, Palladin promotes assembly of non-contractile dorsal stress fibers through VASP recruitment. J. Cell Sci. 127, 1887-98 (2014).

109. C. Furman et al., Ena/VASP is required for endothelial barrier function in vivo. J. Cell Biol. 179, 761-75 (2007).

110. K. A. Rosowski et al., Vinculin and the mechanical response of adherent fibroblasts to matrix deformation. Sci. Rep. 8, 17967 (2018).

111. C. Suzuki et al., ANLN plays a critical role in human lung carcinogenesis through the activation of RHOA and by involvement in the phosphoinositide 3-kinase/AKT pathway. Cancer Res. 65, 11314-25 (2005).

112. P. Hotulainen, P. Lappalainen, Stress fibers are generated by two distinct actin assembly mechanisms in motile cells. J. Cell Biol. 173, 383-94 (2006).

113. P. Sharma et al., Role of dystrophin in airway smooth muscle phenotype, contraction and lung function. PLoS One. 9, e102737 (2014).

114. H. Kamioka, Y. Sugawara, T. Honjo, T. Yamashiro, T. Takano-Yamamoto, Terminal differentiation of osteoblasts to osteocytes is accompanied by dramatic changes in the distribution of actin-binding proteins. J. Bone Miner. Res. 19, 471-8 (2004).

115. N. Sandbo et al., Control of myofibroblast differentiation by microtubule dynamics through a regulated localization of mDia2. J. Biol. Chem. 288, 15466-73 (2013). 
116. H. Honda et al., Cardiovascular anomaly, impaired actin bundling and resistance to Srcinduced transformation in mice lacking p130Cas. Nat. Genet. 19, 361-5 (1998).

117. L. Dolat et al., Septins promote stress fiber-mediated maturation of focal adhesions and renal epithelial motility. J. Cell Biol. 207, 225-35 (2014).

118. K. Sudo et al., SEPT9 sequence alternations causing hereditary neuralgic amyotrophy are associated with altered interactions with SEPT4/SEPT11 and resistance to Rho/Rhotekinsignaling. Hum. Mutat. 28, 1005-13 (2007).

119. A. Cho, M. Kato, T. Whitwam, J. H. Kim, D. J. Montell, An Atypical Tropomyosin in Drosophila with Intermediate Filament-like Properties. Cell Rep. 16, 928-938 (2016).

120. S. Tojkander et al., A molecular pathway for myosin II recruitment to stress fibers. Curr. Biol. 21, 539-50 (2011).

121. L. A. Perkins et al., The Transgenic RNAi Project at Harvard Medical School: Resources and Validation. Genetics. 201, 843-52 (2015).

122. G. Dietzl et al., A genome-wide transgenic RNAi library for conditional gene inactivation in Drosophila. Nature. 448, 151-156 (2007).

123. C. M. Morrison, G. Halder, Characterization of a dorsal-eye Gal4 Line in Drosophila. Genesis. 48, 3-7 (2010).

124. S. Herszterg, A. Leibfried, F. Bosveld, C. Martin, Y. Bellaiche, Interplay between the Dividing Cell and Its Neighbors Regulates Adherens Junction Formation during Cytokinesis in Epithelial Tissue. Dev. Cell. 24, 256-270 (2013).

125. O. Kanca et al., Erratum to Raeppli: A whole-tissue labeling tool for live imaging of Drosophila development (Development, 141, 472-480). Dev. 141 (2014), p. 725.

126. J. Grosshans, E. Wieschaus, A genetic link between morphogenesis and cell division during formation of the ventral furrow in Drosophila. Cell. 101, 523-31 (2000).

127. J. Zanet et al., Fascin is required for blood cell migration during Drosophila embryogenesis. Development. 136, 2557-65 (2009).

128. B. Klapholz et al., Alternative mechanisms for talin to mediate integrin function. Curr. Biol. 25, 847-57 (2015).

129. X. Morin, R. Daneman, M. Zavortink, W. Chia, A protein trap strategy to detect GFPtagged proteins expressed from their endogenous loci in Drosophila. Proc. Natl. Acad. Sci. 98, 15050-15055 (2001).

130. A. M. Vrabioiu, G. Struhl, Fat/Dachsous Signaling Promotes Drosophila Wing Growth by Regulating the Conformational State of the NDR Kinase Warts. Dev. Cell. 35, 737-49 (2015). 


\section{Acknowledgments}

We thank S. Blair, N. Brown, J. Jiang, G. Struhl, the Bloomington, Vienna, Harvard Medical School and Kyoto Stock Centres for reagents; the PICT-IBiSA@BDD imaging facility (ANR-10INBS-04); A. Bardin, F. Graner, E. Hannezo, M. O’Connor, P. Leopold, J-L. Maitre, N. Minc, A. Maugarny-Cales, L. Alpar and E. van Leen for comments. Funding: ANR-MaxForce, ERC Advanced (340784), ARC (SL220130607097), ANR Labex DEEP (11-LBX-0044, ANR-10IDEX-0001-02), NSF IOS1353914 and DMR1056456. J.M.L-G. and F.d.P acknowledge ARC and FRM fellowships, respectively. H.N. and M.S. were supported by NSF Graduate Research Fellowships under grant DGE1256260. D.K.L was supported by Curie Mayent-Rothschild and ICAM senior fellowships. Author contributions: J.M.L-G., D.L., H.N., Y.B. designed the project. I.G., S.P. produced reagents. J.L-G., F.d.P., F.B., O.M. performed experiments. J.M.L-G., H.N, M.S, B.G. developed methods and data analysis scripts. J.M. L-G., H.N., M.S., B.G., F.d.P., F.B. analysed the data. D.L., H.N., M.S. developed theoretical models and performed simulations. J.M.L-G., D.L, H.N., Y.B. wrote the manuscript. Competing interests: The authors declare no competing financial interests. Data and materials availability: All data is available in the main text or the supplementary materials.

\section{Materials and Methods}

Drosophila stocks used in this study are given in Table S2. Methods for genetics, molecular biology, live-imaging, laser ablations and quantifications as well as statistics are described in

Supplementary Materials. The MatLab codes, the Python Jupyter Notebook for plotting and statistical analyses, and the Fiji macros used are available upon request. Physical modeling, computer simulations and associated quantifications are described in the Supplementary Text.

\section{Supplementary Materials:}

Materials and Methods

Supplementary Text

Figures S1-S10

Tables S1-S2

Movies S1-S11

References (40-132) 
Fig. 1. aSF formation in response to tissue mechanical stress.

(A) Pupal dorsal thorax labeled by E-Cadherin:3xGFP (E-Cad:3xGFP) at 26 hAPF and close-up. White line: midline. Dashed box: posterior and central region where aSFs form and where protein distributions and quantifications are reported at $26 \mathrm{hAPF}$ in all figures, unless otherwise stated.

(B) Tissue stress anisotropy estimated by laser ablation (red) and number of aSFs per cell over all cell sizes (blue) as a function of developmental time. $\mathrm{n}$ and $\mathrm{N}$ : numbers of cells and tissue ablations at each time-point, respectively.

(C) E-Cad:3xmKate2 and MyoII:3xGFP distributions at 18 hAPF and 26 hAPF. Arrowheads: aSFs.

(D) Orientation of aSFs (blue) at $26 \mathrm{hAPF}$ and of the tissue's main stress axis (mean \pm STD in red and light red, respectively). n: aSF number.

(E) E-Cad:3xmKate2 and MyoII:3xGFP distributions before ablation and 9.2s after aSF ablation.

Dashed box: ablated region. Arrowheads: positions of the AJs prior to and after aSF ablation.

(F) Recoil velocity upon mock and aSF ablation. n: number of ablations; $p$-value $<10^{-5}$.

(G) Actn:GFP and MyoII:3xmKate2 distributions at 26 hAPF. Arrowheads indicate some aSFs.

(H, H') E-Cad:3xmKate2 and MyoII:3xGFP distributions in the posterior central region in the mirr-G4>LifeAct:GFP (control, H) and mirr-G4>zip ${ }^{D N}: Y F P\left(\mathrm{H}^{\prime}\right)$ animals. Arrowheads: aSFs.

(I-K) m-1 tissue recoil velocity upon a-p ablation (I, n: number of ablations; $p$ value $<10^{-2}$ ) as well as number of aSFs per cell and aSF orientation ( $\mathrm{J}, \mathrm{K}, \mathrm{n}$ : number of aSFs; $p$-value $\left.<10^{-5}\right)$ in control mirr-G4>LifeAct:GFP versus mirr-G4>zip ${ }^{D N}$ :YFP tissues.

(L, L') MyoII:3xGFP distributions at 26 hAPF in an uncompressed tissue (L) and in a tissue compressed along the m-1 axis (L'). Arrowheads: aSFs.

$(\mathbf{M}, \mathbf{N})$ aSF number per cell $(\mathrm{M})$ and orientation $(\mathrm{N})$ in uncompressed tissue versus compressed tissue at 26 hAPF. n: number of aSF per cell; $p$-value $<10-5$.

Scale bars: $50 \mu \mathrm{m}(\mathrm{A}), 5 \mu \mathrm{m}(\mathrm{C}, \mathrm{H}, \mathrm{L})$ and $2 \mu \mathrm{m}(\mathrm{E}, \mathrm{G}$, inset in A).

Kruskal-Wallis (F, I, J, M) and Levene for equality of variances (K, N) tests. ns: not significant. Statistically significant differences $(p$-value $<0.05)$ are indicated by one asterisk. 


\section{Fig. 2. Scaling between cell apical area and aSF number per cell.}

(A) MyoII:3xGFP distribution in $a c t n^{R N A i}$ cells (outlined by an orange dashed line) marked by Caax:tBFP accumulation (not shown) and in control cells. Arrowheads: aSFs.

(B) aSF number per cell in $w^{R N A i}$ control and $a c t n^{R N A i}$ clones at 26 hAPF. n: number of cells; $p$-value $<10^{-5}$.

(C) m-1 cell elongation of $w^{R N A i}$ control and $a c t n^{R N A i}$ clone at $26 \mathrm{hAPF}$. n: number of cells; $p$-value $<10^{-5}$.

(D) m-1 tissue elongation in control versus $a c t n^{R N A i}$ clones in the tissue's central posterior region between 19 and 26 hAPF. N: number of clones; $p$-value $<10^{-5}$.

(E) Recoil velocity (orange) of ablated AJ without prior aSF ablation and after aSF ablation was performed; ( $n$ : number of ablations; $p$-value $<0.05$ ); graph of recoil velocity (blue) upon aSF ablation without prior AJ ablation and after ablation of a neighbouring $\mathrm{AJ}$ ( $n$ : number of ablations; $p$-value $\left.<10^{-5}\right)$. n: number of ablations.

(F) AJ recoil velocity upon ablation in $w^{R N A i}$ control and $a c t n^{R N A i}$ clones at $18 \mathrm{hAPF}$ (ns) and 26 hAPF ( $p$-value $\left.<10^{-4}\right)$. n: number of ablations.

(G) Schematic of the vertex model of a regular cell packing with aSFs under uniaxial stress.

(H) Cell elongation and tension distribution ( $\gamma$, color scale) in the model without or with aSFs at fixed cell size and orientation and at fixed uniaxial tissue stress. Grey cells: differential cell elongation; colored cells: tension magnitude at AJ and individual aSFs (at fixed tension per aSF). Parameter values in Fig. S4I.

(I) Number of aSFs per cell (at fixed tension per aSF) required for cells to remain regular under uniaxial stress versus cell apical area. Parameter values in Fig. S4I.

(J) aSF number per cell versus apical cell size in $w^{R N A i}$ control and $a c t n^{R N A i}$ clones at $26 \mathrm{hAPF}$. n: number of cells. $p$-value $<10^{-5}$.

(K) Changes in cell elongation (percentage) without ablation, upon mock aSF ablation (i.e. ablation next to an aSF), and upon ablation of an increasing number of aSFs in a cell. $p$-value $<10^{-3} . \mathrm{n}$ : number of cells without ablation, with mock ablations or ablations of aSFs.

(L) Cell apical area versus the aSF number per cell in control $w^{R N A i}, \operatorname{survivin}^{R N A i}\left(\operatorname{svn}^{R N A i}\right), \operatorname{aur} B^{R N A i}$, tribbles overexpression $\left(t r b l^{u p}\right)$ and $c d c 2^{R N A i}$ clones. Number of cells $w^{R N A i}(\mathrm{n}=2903), s v n^{R N A i}$ $(\mathrm{n}=872), \operatorname{aurB}^{R N A i}(\mathrm{n}=402) \operatorname{trbl}^{U P}(\mathrm{n}=356)$ and $c d c 2^{R N A i}(\mathrm{n}=227)$.

(M) Difference in m-1 cell elongation between $a c t n^{R N A i}$ cells and $w^{R N A i}$ control clones as a function 
of apical cell size at different hAPF. n: minimum number of cells at each hAPF and condition. $p$-value $<10^{-4}$

Scale bar: $5 \mu \mathrm{m}$.

Kruskal-Wallis without (B, C, E, F), with Conover post hoc (K), Ancova (J, M) and mixedANOVA (D) tests. 


\section{Fig. 3. Hippo component clustering and aSF formation.}

(A-B) Jub:GFP (A), Wts:GFP (B) and MyoII:3xmKate2 (A,B) distributions at 26 hAPF. Arrowheads: aSF tips.

(C) Jub:GFP and Wts:GFP ratio ${ }^{\text {in/out }}$ of clusters at 18 hAPF (low stress) and 26hAPF (high stress). $\mathrm{n}$ : number of cells; $p$-values $<10^{-5}$ for the comparison between $18 \mathrm{hAPF}$ and $26 \mathrm{hAPF}$ for both Jub:GFP and Wts:GFP.

(D-D') Jub:mKate2 and Wts:GFP distributions at 18 hAPF (low stress, D) and 26 hAPF (high stress, D'). Arrowheads: Jub:mKate2 and Wts:GFP co-clusters.

(E) Jub:mKate2 and MyoII:3xGFP distributions during aSF formation. $\mathrm{t}=0$ corresponds to aSF nucleation. Arrowheads: aSF.

(F) Jub:GFP distribution before and after $(\mathrm{t}=90 \mathrm{~s})$ ablation (dashed box) of the MyoII:3xmKate2 labelled aSF. Arrowheads: cluster prior to and after ablation.

(G) Jub:GFP cluster intensities in mock ablated aSF (control, blue) and upon aSF ablation (red). n: number of clusters; $p$-value $<10^{-3}$ after timepoint $30 \mathrm{~s}$.

(H) Jub:GFP and Wts:CitFP ratio ${ }^{\text {in/out }}$ of clusters in $w^{R N A i}$ control and $a^{2 t n^{R N A i}}$ clones at $26 \mathrm{hAPF}$. n: number of cells; $p$-values $<10^{-5}$ for Jub:GFP and Wts:CitFP

(I) ban-nls:GFP normalized intensity in $w^{R N A i}$ control and $a c t n^{R N A i}$ clones at 18 and 26hAPF. $\mathrm{N}$ : number of animals; 18 hAPF: ns; 26 hAPF: $p$-value $<10^{-3}$.

(J) Fraction of cells that divide between 18 and 26 and between 26 and 34hAPF in pnr-G4>w $w^{d s R A}$ control and $p n r-G 4>a c t n^{d s R N A}$ tissues; prior to 26hAPF: ns; after $26 \mathrm{hAPF}$ : $p$-value $<0.05$. N: number of animals.

Scale bars: $2 \mu \mathrm{m}$.

Kruskal-Wallis without (C, H, I), with Conover post hoc $(\mathrm{G})$ and One-tailed Wilcoxon signed rank (J) tests 


\section{Fig. 4. Hippo scaling as a function of cell apical area.}

(A) ban-nls: GFP and Ubi-nls: GFP intensity versus apical cell area. n: cell numbers. $p$-value $<10^{-5}$.

(B) ban-nls:GFP intensity versus Jub:mKate2 ratio $^{\text {in/out }}$ of cluster. n: number of cells.

(C) Wts:CitFP and Jub:GFP ratio ${ }^{\text {in/out }}$ of cluster versus apical cell area. n: number of cells.

(D, E) Jub:GFP (D) and Wts:CitFP (E) ratio ${ }^{\text {in/out }}$ of cluster versus apical cell size in $w^{R N A i}$ control and $a c t n^{R N A i}$ clones. n: number of cells; $p$-value $<10^{-4}$.

(F) Fraction of cells that divide between $22 \mathrm{hAPF}$ and $34 \mathrm{hAPF}$ as a function of apical cell size in pnr-G4> $w^{d s R N A}$ control and pnr-G4>actn ${ }^{d s R N A}$ tissues. N: number of animals; $p$-value $<0.05$.

Ancova for difference in regression slopes (A,C-E) and Mixed-ANOVA (F) tests. 
Fig. 5: TCJs contribute to a scaling between cell area and aSF number.

(A) E-Cad:3xmKate2 and MyoII:3xGFP distributions as an aSF nucleates ( $\mathrm{t}=0 \mathrm{~min}$, circles: TCJs), peels from the cortex ( $\mathrm{t}=2$ to $14 \mathrm{~min}$ ), moves towards TCJs ( $\mathrm{t}=18$ to $22 \mathrm{~min}$ ), briefly stalls at TCJs $(\mathrm{t}=34 \mathrm{~min})$ and breaks $(\mathrm{t}=42 \mathrm{~min})$. Red arrowhead: nucleating TCJ; yellow arrowheads: aSF tips; blue arrowheads: breaking TCJs. Open yellow arrowheads: aSF tip positions at the time of breakage.

(B) Illustration of an aSF nucleating at a nucleating TCJ (red arrowhead) and peeling from the cortex until the aSF tips reach breaking TCJs (blue arrowheads) and of the TCJ opening angle $\alpha$ and TCJ bisector orientation $\theta$ with respect to the a-p axis. Orange arrows indicate the TCJ bisector, which makes an angle $\theta$ with the a-p axis $\left(\theta \approx 5^{\circ}\right.$ for the nucleating TCJ at right; $\theta \approx 80^{\circ}$ for the breaking TCJ at bottom).

(C) aSF nucleation (red) and breakage (blue) events at TCJs. n: total number of aSFs.

(D) Experimental (grey) and model-predicted (green) aSF number per cell versus apical area. The model explains approximately 75 percent of the variance of aSF number with cell apical area. n: number of cells.

(E) Schematic based on a Jub:GFP image illustrating the positions of the control (white), border (brown) and bulk (blue) $t r b l^{U P}$ cells.

(F-I) Nucleating TCJs per cell (E, $p$-value $<10^{-5}$ ), aSF number per cell ( $\mathrm{G} p$-value $<10^{-2}$ controlling for cell apical area differences), predicted aSF lifetime $(\mathrm{H})$, predicted aSF number per cell (I, $p$ value $<10^{-2}$ controlling for cell apical area differences) in $t r b l^{U P}$ border and bulk cells. n: number of cells.

(J) Schematic of elongated (top) and ortho-elongated (bottom) cells.

(K-O) aSF number per cell (K, $p$-value $\left.<10^{-5}\right)$, nucleating TCJs per cell (L), cell apical area (M), predicted aSF lifetime $\left(\mathrm{N}, p\right.$-value $\left.<10^{-5}\right)$, predicted aSF number per cell $\left(\mathrm{O}, p\right.$-value $\left.<10^{-5}\right)$ in elongated and ortho-elongated cells. n: number of cells.

Scale bar: $2 \mu \mathrm{m}$.

Kruskal-Wallis tests (F-I, K-O). 


\section{Science МIAAAS}

\section{Supplementary Materials for}

\section{Apical stress fibers enable a scaling between cell mechanical response and area in epithelial tissue}

Jesús M. López-Gay, Hayden Nunley, Meryl Spencer, Florencia di Pietro, Boris Guirao, Floris Bosveld, Olga Markova, Isabelle Gaugue, Stéphane Pelletier, David K. Lubensky and Yohanns

Bellaïche

Correspondence to: dkluben@umich.edu; yohanns.bellaiche@curie.fr

\section{This PDF file includes:}

Materials and Methods

Supplementary Text

Figs. S1 to S10

Tables $\mathrm{S} 1$ to $\mathrm{S} 2$

Captions for Movies S1 to S11

Other Supplementary Materials for this manuscript include the following:

Movies S1 to S11 


\section{Materials and Methods}

Fly stocks and genetics

Supplementary Table 2 lists the Drosophila melanogaster stocks used in this study as well as the associated references. Loss-of-function and gain-of-function experiments were carried out using the FLP/FRT, the Gal4/UAS and Gal4/Gal80\% $/$ UAS systems (40-43). Somatic clones were induced in the second instar or third instar larval stages by heat-shock (HS) at $37^{\circ} \mathrm{C}$. When comparing gene function at $18 \mathrm{hAPF}$ or $26 \mathrm{hAPF}$, the timing after clone induction (hACI) was identical to avoid possible confounding effects due to protein perdurance.

\section{Molecular biology}

The $a c t n^{I G I}$ and $a c t n: G F P$ alleles were generated by CRISPR/Cas9-mediated homologous recombination at their endogenous loci, using the vas-Cas9 line (44). To generate the $a c t n^{I G I}$ and actn:GFP alleles by CRISPR/Cas9-mediated homologous recombination, guide RNAs were cloned into the $p C F D 5$ : U6:3$t:: g R N A$ vector (45). For the $a c t n^{I G I}$ allele, which deletes the amino-acid (aa) 841 to 2754 , the following guide RNAs were used: 5'-TGCCTTGCGCTCTTACAAGTCGG -3', 5'TGCTGAAGGACATGTAGTCCAGG 5', 5'-GGCAAAAACTGGGAAAGCACAGG-3', 5'CCAAAACCGCTTGACTTCAATGG-3', 5'-AACTGGGAAAGCACAGGCCAAGG-3', , 5'CTTGACTTCAATGGAGTCCTTGG-3' ; while for the actn:GFP allele we used the following ones: 5'GACCGACTTGTAAGAGCGCAAGG-3' and 5'-TGCAGTCCTTCGGATGCGGGTGG-3'. Homology sequences were cloned into homologous recombination vectors harboring a hs-miniwhite cassette either flanked by two loxP sites $(9,46)$ and an N-terminal GFP sequence for GFP tagging (vector and map available upon request) or flanked by two loxP to generate the actn $^{I G I}$ sites and two attP sites for deletion allele $(9,46)$. The two homologous regions (HR1 and HR2) flanking the site of CRISPR/Cas9 cuts were cloned using the following primers: (i) for the $\operatorname{acth}^{I G I}$ : (HR1) 5'-CCGGGCTAATTATGGGGTGTCGCCCTTCGCGCATCCGCAACTGCCAGATGC-3' 5'-ACTCAAAGGTTACCCCAGTTGGGGCACTACGGGATTAGAATGCTCTAGCGAATTTGC-3'; (HR2) 5'- ACTCAAAGGTTACCCCAGTTGGGGCACTACAAGGACTGCATCGGCGAACCC-3' and 5'-ACTCAAAGGTTACCCCAGTTGGGGCACTACAAGGACTGCATCGGCGAACCC-3'; (ii) for actn:GFP : : (HR1) 5'- TATGGGGTGTCGCCCTTCGGGTCTCTAGTTATTGTGAAGCAGCACCAGATCCC-3' and 5'- ACTGCCTGAAGAACCGCTGGACCCCGAACTCAAGTCGGTCTCGCCGTAGAG-3’;

(HR2) 5'- GGAAGTGGTAGCTCAGGGTCTAGTGGATAGAAGGACTGCATCGGCGAACCC-3' and 5'- GCCCTTGAACTCGATTGACGCTCTTCGTCCGCCTGTGGGTGAATGTAGTGTGTTT-3'.

Jub:mKate2 was built based on the rescue construct described in (47) and was cloned by PCR ligation in pCaSpeR4 using the following primers: (Jub genomic region) 5'TTAACGTTCGAGGTCGACTCTAGAGGATCCGCGTTGTTGTGGCTTCCTATGCTAAC-3', 5GTGCATGTTCTCCTTAATCAGCTCGCTCACTCCCATATACTGGTACGAAGCGCAG-3' and (mKate2) 5'- GTGAGCGAGCTGATTAAGGAGAAC-3' GCCTCTCCCAGCCACACTCCGTTCCGCTTATCTGTGCCCCAGTTTGCTAGGG-3’'

Cry2Olig:ChFP and Cry2Olig:ChFP:Jub were cloned in the pCaSpeR4 Ubiquitin promotor transgenesis vector (pUbi) in the following way: the pUbi-Cry2Olig:ChFP was first generated by cloning in the pUbi vector a PCR fragment of the Cry2 PHR Olig-mCherry (E490G) amplified from pCMV_Cry2PHROligmCherry(E490G) using 5 ATCCCCCGGGCTGCAGGAATTCACAGATCTATGAAGATGGACAAAAAGACTATAGTTTGG-3' and 
CATGCCG-3'. The genomic region of the jub gene (47) from the ATG to the STOP codons was then cloned in the resulting vector using the TCCACCGGCGGCATGGACGAGCTGTACAAGGGTTCGGGCTCGGGTAGTATGACCACCCAGCG GACGCAG-3' GCGCTCAGCTGGAATTGGGGTACGTCTAGATTATCCCATATACTGGTACGAAGCGCAG-3' for PCR amplification and addition of a GSGSGS protein linker between mCherryFP and Jub. All embryo transgenesis injections were performed by Bestgene.

\section{Live imaging microscopy}

Pupae were prepared for dorsal thorax live imaging as described previously $(4,9)$. Unless otherwise stated samples were imaged at $25^{\circ} \mathrm{C}$ or $29^{\circ} \mathrm{C}$ with an inverted confocal spinning disk microscope from Nikon or Zeiss, using either 60x NA1.4 OIL DIC N2 PL APO VC, 63x NA1.4 OIL DICII PL APO or 100x NA1.4 OIL DIC N2 PL APO VC objectives and sCMOS Hamamatsu camera. Additionally, images and movies were obtained with a confocal microscope Carl Zeiss (LSM880 NLO,) with 40x NA1,3 OIL DICII PL APO (UV) VIS-IR (optical zoom 2.5X) or a 63x NA 1.4 OIL DICII PL APO objective (optical zoom 5X or 7.5X). This confocal microscope was also used for laser ablations (see Laser ablations section) since it is equipped with a two-photon Ti:Sapphire laser (Mai Tai DeepSee, Spectra Physics). In several experiments, a single image was acquired at $26 \mathrm{hAPF}$ using confocal spinning disk or scanning microscopy. To record the long timescale dynamics of aSFs, pupae expressing E-Cad:3xmKate2 and MyoII:3xGFP were imaged for 8 - 10 $\mathrm{h}$ every 5 min using confocal spinning disk microscopy. To image the short timescale dynamics of aSFs, animals expressing E-Cad:3xmKate 2 and MyoII:3xGFP were imaged every 30 seconds or every 2 min using confocal spinning disk microscopy.

\section{PDMS tissue compression}

22 hAPF pupae were glued on a pre-stretched PDMS membrane mounted on a custom-made stretcher (Markova et al., in preparation). By releasing the PDMS membrane using a micrometric screw while imaging the pupa, a compressive strain of $20 \%$ was applied in the plane of the tissue. Images were acquired at $26 \mathrm{hAPF}$ through the PDMS membrane on an inverted confocal spinning disk microscope from Nikon using a dry Nikon 40x NA 0.95 AIR PL APO objective.

\section{Image processing and segmentation}

Unless otherwise stated, images were denoised using the Fiji Gaussian Blur 3D plugins to improve signalto-noise ratio, and time-lapse movies were bleach-corrected using the Fiji Bleach Correction Histogram Matching function. For single time-point z-stack images and time-lapse movies, z-stacks were projected using a custom MATLAB code $(10,48)$. The custom MATLAB code automatically determines an apical zmap using the E-Cad:3xmKate2, E-Cad:GFP, Jub:GFP or Jub:mKate2 signal. The apical z-map can then be used to project the relevant z-sections above and below the AJ. Maximum projections were used for aSF number quantification, aSF dynamics and segmentation, whereas average projections were used for quantification of fluorescence intensity. 2D cell segmentation and tracking was achieved using previously published MATLAB codes (10). Segmentation errors were corrected through several iterations between manual and automatic segmentation rounds (10). 3D cell segmentation was performed in Fiji by manually delineating the cell contour along the cell apical-basal axis using the membrane PH:GFP marker. To quantify the nls:GFP nuclear signal, two distinct image processing pipelines were applied. If an AJ marker was also acquired with the nls:GFP signal, the AJ z-map (see above) was used to compute an average projection of the nls:GFP signal between $0 \mu \mathrm{m}$ to $10 \mu \mathrm{m}$ below the AJ z-map. We verified that this procedure projects the entire nls:GFP signal. In the absence of an AJ marker, the range of apical-basal positions of the nls:GFP signal was defined as follows: the z-stacks were filtered by a 2D Gaussian Blur of 40 sigma using Fiji, and the resulting stacks were resliced to obtain y-stacks along the apical-basal axis. The y-stacks were 
binarized using Fiji Mean threshold. The binarized y-stacks were then resliced back to binarized z-stacks. The resulting z-stacks were then manually corrected and used to define the apical-basal position of the nlsGFP nucleus signal. The binarized z-map was then used to perform an average projection of the nls:GFP signal. Each nucleus was manually segmented in Fiji to determine its average nls:GFP signal.

\section{Quantification of number of aSFs and their orientations.}

In order to rigorously compare the number of aSFs in different experimental conditions, the region harboring aSFs in the posterior and medial region of the tissue (black dashed box in Fig. 1A) was defined based on the positions of the two posterior scutellar macrochaetae as precise landmarks. The region enriched in aSFs (aSF region) was defined as a box located $22 \mu \mathrm{m}$ anterior to the two posterior scutellar macrochaetae, centered on the midline and of size $122.5 \mu \mathrm{m}$ (anterior-posterior, a-p axis) by $127.5 \mu \mathrm{m}$ (medial-lateral, $\mathrm{m}-1$ axis). Quantifications of aSF number per cell were performed on projections ( $7 \mathrm{z}$-slices, $3 \mu \mathrm{m}$ ) using MyoII:3xGFP or MyoII:3xmKate2 to label aSFs. aSFs were manually marked in Fiji, and cell contours were obtained by E-Cad:3xmKate2, MyoII:3xGFP or Jub:3xmKate2 signal segmentation. Each marked aSF was then automatically assigned to a given cell using a custom MATLAB code. To compare the scaling between number of aSFs per cell and either cell apical area or cell volume, aSF number, cell apical area and volume were determined in Fiji using manual aSF counting, 2D cell contour segmentation and the 3D cell segmentation, respectively. Subsequent aSF quantifications and data analysis were performed using custom MATLAB and Python codes (see below). In wt tissue, the quantification of cell apical area versus aSF number per cell was independently checked by 3 researchers. aSF orientations were obtained by manually segmenting each aSF in Fiji and then determining its orientation using the Fiji Analyze Particles tool. To test the relationship between aSF orientation and tissue stress anisotropy, aSF orientation and the tissue's inferred local main stress axis were measured in two additional bilaterally symmetric regions in the scutum (green dashed boxes in Fig. S1A'). Rigorously defined based on macrochaetae positions, these two regions (green dashed boxes in Fig. S1A') correspond to $318 \mu \mathrm{m}$ by $68 \mu \mathrm{m}$ rectangles, each laterally centered around the posterior dorsocentral macrochaetae and anterior-posteriorly positioned $5 \mu \mathrm{m}$ posterior to the same macrochaetae.

To analyze the role of lateral tissue contraction on aSF number in the central domain of the tissue, the mirrGal4 (mirr-G4) driver was used to express the dominant-negative transgene for MyoII heavy chain (UASzip ${ }^{D N}$ :YFP, (49)) or the F-Actin marker as control (UASLifeAct:GFP) in the lateral domain of the dorsal thorax. To restrict the expression of $z i p^{D N}: Y F P$ or LifeAct:GFP to the late pupal stage, the animals were initially kept at $18^{\circ} \mathrm{C}$, shifted to $25^{\circ} \mathrm{C}$ at $1 \mathrm{hAFP} \pm 1$ hour and imaged at $26 \mathrm{hAPF}$. To analyze the impact of tissue compression on aSF number, pupal epithelial tissues were compressed (see PDMS Tissue Compression), and aSF numbers were compared in compressed and non-compressed animals. aSF number per cell was also quantified at 26 hAPF (i) in Gal4 flip-out clones labeled by the expression of UASCaax:tBFP and expressing the relevant dsRNA or transgenes under UAS control. In Fig. 2A-C, each clone of $w^{R N A i}$ and $a c t n^{R N A i}$ has, on average, $121 \pm 26$ cells and $120 \pm 33$ cells (mean \pm SEM), respectively; (ii) in acth $^{I G l}$ mutant clones labelled by loss of nls-GFP expression.

To test whether an increase in mechanical stress would be sufficient to promote aSF formation, Myosin-IIphosphatase-regulatory-subunit-loss-of-function clones $\left(\mathrm{mbs}^{R N A i}\right)$ were generated to locally increase MyoII activity and junctional tension $(50,51)$. The number of aSFs per cell in the two layers of wt cells surrounding the $m b s^{R N A \mathrm{i}}$ clones was then compared to the number of aSFs per cell in the two layers of wt cells surrounding $w^{R N A i}$ control clones at $20 \mathrm{hAPF}$. 


\section{Measurements of aSF dynamics}

To study aSF dynamics on the timescale of minutes, E-Cad:3xmKate 2 and MyoII:3xGFP time-lapse movie stacks were projected $(7 \mathrm{z}$-slices, $3 \mu \mathrm{m})$. For each tracked cell, the E-Cad:3xmKate2 signal was used to define the cell contour and the positions of the cell's tricellular junctions (TCJs). At every time-point from nucleation to breakage, the MyoII:3xGFP labelled aSFs were manually annotated at both tips. aSF nucleation was defined as the time-point when a line of MyoII:3xGFP signal is clearly separated from the MyoII:3xGFP junctional cortical signal. aSF breakage was defined as the time-point at which the aSF no longer connects to the two opposing junctions. aSF nucleation rates and aSF lifetimes were studied in control and $a c t n^{R N A i}$ cells. To determine the position of each aSF relative to TCJs, based on the E-Cad:3xmKate2 signal, the positions of nearby TCJs were manually annotated at the aSF's nucleation time, midlife time and breakage time. "Nucleating TCJs" and "breaking TCJs" were also annotated and defined as the TCJs at which the aSF nucleates and breaks, respectively. The position of each aSF's "nucleating TCJ" was tracked during the entire aSF's lifetime. Additionally, aSF velocities were measured from the position where the aSF nucleates to its breakage site.

\section{Protein clustering}

To determine the ratio of Jub and Wts integrated intensity in clusters relative to that elsewhere along the junction (ratio ${ }^{\text {in/out }}$ of cluster, see also Fig. S5E-E"'), z-stacks of single cells expressing Jub:mKate2, Wts:GFP or Wts:CitFP were cropped and sum-projected (17 z-slices — corresponding to $3.2 \mu \mathrm{m}$ — centered around the AJs). Three fluorescence intensity measurements were then performed: (i) The mean fluorescence intensity in cluster (IC). Clusters were automatically identified using the Fiji FindFoci plugin (52) (Fig. S5E') using the following parameters: background, 2; search, 0.15; search method, Fraction of peak - background; peak, 0.15; maximum peaks, 50; fraction, 0.50; minimumsize, 5. The resulting mask was used to measure the intensity in each cluster (Ic) and the area occupied by each cluster (Ac); (ii) The total integrated intensity along the AJ, (Iaj). The AJ cell contour was selected using Fiji segmented line tool (blue, in Fig. S5E"',) and used to measure (Iaj) and the total area of the cell junction (Aaj) and; (iii) The background fluorescence intensity $(I b)$ was measured by selecting the apical medial region of the cells (red, Fig. S5E"') and measuring the average intensity level. The signal in cluster was defined as in cluster = $\frac{\sum_{\text {each cluster }}((I c-I b) * A c)}{(I a j-I b) * A a j}$ and the signal on the junction as out ${ }^{\text {cluster }}=1-$ in $^{\text {cluster }^{\text {. The ratio }}}{ }^{\text {in/out }}$ of cluster

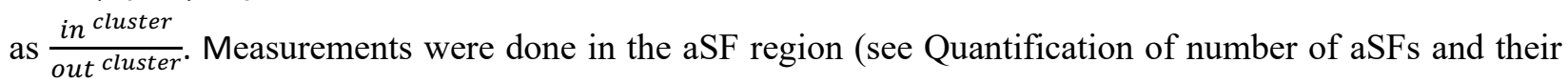
orientations for description of the region) as well as in a region devoid of aSFs at both 18 hAPF and 26 $\mathrm{hAPF}$ in control tissues as well as in $w^{R N A i}, a c t n^{R N A i}, s v n^{R N A i}$ and $a u r B^{R N A i}$ clones in the aSF region.

\section{Optogenetics}

Pupae expressing Cry2Olig:ChFP:Jub or Cry2Olig:ChFP were kept at $18^{\circ} \mathrm{C}$ in the dark and mounted as in $(4,9)$ but under red light exposure. Cry2Olig:ChFP light activation was performed by $491 \mathrm{~nm}$ laser illumination, and the dynamics of Cry2Olig:ChFP or Cry2Olig:ChFP:Jub were recorded using the $561 \mathrm{~nm}$ laser line. All optogenetic experiments were performed in a region devoid of aSFs, located between the two dorsocentral macrochaetae and adjacent to the midline. Clustering was induced by two initial steps of high blue laser illumination (see below for different activation times) and then maintained at low laser power for GFP imaging. During the $491 \mathrm{~nm}$ illumination, Wts:GFP, Jub:GFP, MyoII:GFP were imaged every 50s. To analyze whether the $491 \mathrm{~nm}$ illumination of Cry2Olig:ChFP:Jub or of Cry2Olig:ChFP modulates the level of the dIAP l-nls:GFP transcriptional Yki reporter, the nls:GFP signal intensities were compared in "dark" and "light" experimental conditions: (i) "light" condition : upon an initial $491 \mathrm{~nm}$ illumination, the dIAP1nls:GFP signal was acquired by $491 \mathrm{~nm}$ illumination every $5 \mathrm{~min}$ for $4 \mathrm{~h}$; (ii) "dark" condition: a single image 
was acquired using the $491 \mathrm{~nm}$ laser at the beginning of the experiments, and then animals were kept in dark for $4 \mathrm{~h}$ until a second acquisition of the nls:GFP signal was performed using the $491 \mathrm{~nm}$ laser line. Upon acquisition, the stacks were average-projected, and the average nls:GFP signal and background were measured. The changes in $d I A P 1$-nls:GFP expression due to illumination were measured as:

$\frac{d I A P-n l s: G F P^{\text {light }, 4 h}(\text { Cry2Olig:ChFP:Jub })}{d I A P-n l s: G F P^{\text {light }, 0 h}(\text { Cry2Olig:ChFP:Jub })} /<\frac{\text { dIAP-nls:GFP }}{\text { light,4h }(\text { Cry2Olig:ChFP })}>$

corrected for background signals, and similarly for dark condition.

\section{Laser ablations}

Tissue recoil velocity. To measure tissue recoil velocity upon laser ablation, E-Cad:3xGFP or Jub:GFP labelled tissue was imaged using a two-photon laser-scanning microscope (LSM780 or LSM880 NLO, Carl Zeiss) equipped with a 40x NA1,3 OIL DICII PL APO (UV) VIS-IR objective (zoom 2.5X) in single-photon bidirectional scan mode lasting $\delta \mathrm{t}=756 \mathrm{~ms}$ every $5 \mathrm{~s}$. A rectangular region (length $0.83 \mu \mathrm{m} \mathrm{x}$ width $41.5 \mu \mathrm{m}$ ) was ablated at $\mathrm{t}=0 \mathrm{~s}$ using the Ti:Sapphire laser (Mai Tai DeepSee, Spectra Physics) typically set at 30\% power at $805 \mathrm{~nm}$ with $<100 \mathrm{fs}$ pulses with a $80 \mathrm{MHz}$ repetition rate. Ablations were performed parallel or orthogonal to the midline region to measure tissue recoil velocity along the a-p or $\mathrm{m}-\mathrm{l}$ axis, respectively. Upon ablation, images were acquired every $5 \mathrm{~s}$ for 60 seconds. Recoil velocity was estimated by measuring the displacement of the tissue at the edge of the ablated region between $\mathrm{t}=0 \mathrm{~s}$ and $25 \mathrm{~s}$. The ablations were performed in pupae at different time-points between $14 \mathrm{hAFP}$ and $28 \mathrm{hAPF}$ to determine the dependence of the recoil velocity on developmental time. In a similar manner, tissue recoil velocities were measured in $p n r-G 4>a c t n^{d s R N A}$ and $p n r-G 4>w^{d s R N A}$ tissues at $26 \mathrm{hAPF}$. Tissue stress anisotropy was defined as the ratio of the mean recoil velocity along the $\mathrm{m}-1$ axis to the mean recoil velocity along the a-p axis (Fig. 1B and Fig. S1C). The error on the difference in mean elongations was obtained through standard error propagation of the SEMs of the two means at each time-point. Error propagation was performed by using the uncertainties package in Python (53).

aSF and AJ recoil velocities. To measure junctional recoil velocity upon aSF or AJ ablation, tissues expressing E-Cad:3xmKate2 and MyoII:3xGFP were imaged at $26 \mathrm{hAPF}$ as described above except that images were acquired at the following time-points: $-1.1 \mathrm{~s},-0.1 \mathrm{~s}, 0.65 \mathrm{~s}, 1.3 \mathrm{~s}, 2.2 \mathrm{~s}, 3.2 \mathrm{~s}, 4.2 \mathrm{~s}, 5.2 \mathrm{~s}, 6.2 \mathrm{~s}, 7.2 \mathrm{~s}$, $8.2 \mathrm{~s}$ and 9.2s. Ablation was performed at $\mathrm{t}=0$. Regions to be ablated (ROI) were precisely defined to cover an aSF or an AJ (see Fig. S1D and Fig. S3J for aSF and AJ ablation schematics, respectively). In the case of a mock ablation, the ROI was set adjacent to an aSF. The ROI was ablated using the Ti:Sapphire laser (Mai Tai DeepSee, Spectra Physics) typically at $35 \%$ power at $805 \mathrm{~nm}$ with $<100$ fs pulses with a $80 \mathrm{MHz}$ repetition rate. aSF recoil velocity was deduced from the displacement (between $t=0$ and $t=1.3 \mathrm{~s}$ ) of the junctions to which the aSF was anchored. AJ recoil velocity was calculated by the displacement of the two vertices of the $\mathrm{AJ}$ between $\mathrm{t}=0$ and $\mathrm{t}=1.3 \mathrm{~s}$. Changes in cell elongation caused by ablations were compared to the cell elongation variations measured during an interval of approximately $9.5 \mathrm{~s}$ in the absence of ablations.

In the cases of an aSF ablation followed by an AJ ablation or of an AJ ablation followed by an aSF ablation, only aSFs and AJs oriented between $72^{\circ}$ and $118^{\circ}$ relative to the a-p axis were ablated. The ablation of the second MyoII structure, either aSF or AJ, was performed approximately $30 \mathrm{~s}$ following the initial ablation; this time interval between ablations is the amount of time necessary for measuring the recoil velocity associated with the first ablation, readjusting the microscope's ablation settings and ROIs, and launching the second ablation. This experimental constraint on the time between ablations could conceivably affect our tension measurements if the second structure to be ablated reorients in the time between ablations or if a healing process (i.e., the beginning of the closure of the wound) occurs between ablations. Based on experimental data, we argue that neither healing nor reorientation of the second structure to be ablated occurs in the time between ablations. To test whether an aSF reorients after the first (AJ) ablation and before its own ablation, we measured the orientation of the aSF before AJ ablation and just before the aSF ablation 
(i.e., 30s later). The change of aSF orientation is $2^{\circ} \pm 2^{\circ}$ (mean $\pm \operatorname{std}, n=51$ and Movie S5), and no correlation exists between the magnitude of the aSF reorientation and the subsequently measured tension of the aSF $\left(\mathrm{R}^{2}=0.016\right)$. Similarly, for the reorientation of the AJ immediately after an aSF ablation, the average reorientation is $3^{\circ} \pm 2.7^{\circ}(\mathrm{n}=37$ and Movie S6), and no correlation exists between reorientation and the subsequently measured magnitude of AJ tension $\left(\mathrm{R}^{2}=0.009\right)$. Regarding healing processes, Movie S5 and Movie S6 show that the second ablation is performed before any healing process at the AJ or the aSF is observed. Accordingly, in another set of AJ ablations where we recorded the dynamics on a longer timescale, the first sign of a healing process is observed $120 \pm 50 \mathrm{~s}(\mathrm{n}=20)$ post-ablation, and no healing process is observed after aSF ablation $(\mathrm{n}=40)$. AJ ablation was also performed in $a c t n^{R N A i}$ and $w^{R N A i}$ flip-out clones at $18 \mathrm{hAPF}$ and $26 \mathrm{hAPF}$ in tissue labeled by Jub:GFP and MyoII:3xmKate2.

Jub and Wts cluster dynamics upon aSF ablation: To study the dynamics of Jub:GFP, Wts:GFP or Wts:CitFP clusters upon aSF ablation, aSFs labelled by MyoII:3xmKate2 were ablated as described above except that a single time-point was acquired prior to ablation and that images were acquired every 30s upon aSF ablation. In addition, in cases where the given aSF was connected to another aSF, both aSFs were ablated. Upon acquisition, the intensities of Jub or Wts clusters were measured as follows: a ROI corresponding to the cluster of Jub:GFP, Wts:GFP or Wts:CitFP prior to ablation was manually delineated using the Fiji Polygon selection tools; the average fluorescence intensities in the ROI were then measured at each time-point by manually tracking the position of the cluster at each time-point. The average background fluorescence intensity was determined by averaging 5 intensity measurements performed in the medial region of the surrounding cells. Similar results were obtained for Wts:GFP and Wts:CitFP (not shown).

\section{Cell and tissue elongations, stress inference and rate of cell division.}

Cell elongation. Upon cell segmentation, we determined the cell elongation as follows. First, we determined the geometric center of the cell $\left(x_{c}, y_{c}\right)$. Then, for each pixel $i$ within the cell, we computed $\Delta x_{i}=x_{i}-x_{c}$, $\Delta y_{i}=y_{i}-y_{c}$ and the cell inertia matrix as:

$$
I=\left[\begin{array}{ll}
I_{x x} & I_{x y} \\
I_{x y} & I_{y y}
\end{array}\right]=\sum_{\text {pixels within cell }}\left[\begin{array}{cc}
\Delta x_{i}^{2} & \Delta x_{i} \Delta y_{i} \\
\Delta x_{i} \Delta y_{i} & \Delta y_{i}^{2}
\end{array}\right] .
$$

The cell elongation along the m-l axis was defined as the ratio between cell inertia matrix component $I_{y y}$ (m-l axis component) and the $I_{x x}$ component (a-p axis component). Ortho-elongated cells are defined as those with $I_{x x}>I_{y y}$ and elongated cells as those with $I_{y y}>I_{x x}$.

Upon aSF ablation (at $\mathrm{t}=0 \mathrm{~s}$ ), the change in cell elongation was measured as the difference between the cell elongation at $\mathrm{t}=-0.1 \mathrm{~s}$ and $\mathrm{t} \approx 9.5 \mathrm{~s}$. The differences in cell elongation between $\operatorname{actn}^{R N A i}$ and $w^{R N A i}$ cells were calculated for each cell apical area bin at the indicated developmental times. The errors of the mean elongation differences were obtained by propagation of the standard errors of the $a c t n^{R N A i}$ and $w^{R N A i}$ means for each cell apical area bin. Error propagation was performed by using the uncertainties package in Python (53). A $\pm 1.5 \mathrm{IQR}$ (InterQuartile Range) filter was applied to exclude cells with outlier values of cell elongation. This filter was applied to both $w^{R N A i}$ and $a c t n^{R N A i}$ cells.

Tissue elongation, rate of cell division and stress inference: Upon cell segmentation and tracking, tissue elongation rate, cell division rate, fraction of dividing cells and junctional stress were determined in wt and mutant clones. Tissue properties can be heterogeneous in this region of the tissue, and clone position and size cannot be controlled, thereby making the comparison between a mutant clone's behavior in our ROI and that of its control challenging. Therefore, for each mutant clone we analyzed, we first cropped it to its intersection with the ROI, and we applied this exact same zone of analysis (ZOA) onto the corresponding wt control tissue to make sure that the exact same region would be analyzed in the mutant clone and its 
corresponding control. This was made possible thanks to prior time and space registration of each movie as described in (10). In Fig. 2D, for control and $a c t n^{R N A i}$ clones, each clone contains, on average, $71 \pm 16$ cells and $85 \pm 24$ cells (mean \pm SEM), respectively. Then, the rate of tissue elongation along the $m-1$ axis $(y)$ at a given time point in this ZOA was directly determined by calculating the slope of the line fitting the data points corresponding to the measured cell velocity along the $\mathrm{m}-1$ axis $\left(V_{y}\right)$ plotted against the cell position along this axis (y); $\partial_{y} V_{y}=\dot{\varepsilon}_{y y}$, as described in (54). Then, the cumulative tissue elongation along the m-1 axis over a time period of T could be calculated: $\int_{0}^{T} \dot{\varepsilon}_{y y} d t, d t$ being the time duration between two frames. The cell division rate $\left(\mathrm{h}^{-1}\right.$, Fig. S5V) in a ZOA at a given time was determined by counting the number of divisions occurring over an hour normalized by the initial number of cells in the ZOA. The fraction of dividing cells in Fig. 3J is the number of cells dividing in a given time range divided by the initial number of cells in the ZOA. The fraction of dividing cells in Fig. $4 \mathrm{~F}$ is the ratio between the number of cells achieving a second division and the total number of cells in each size bin. Each cell size is determined by averaging its mother cell size over $6 \mathrm{~h}$. Inference of the junctional stress in the ZOA was performed as described in (10). The region used in each animal contained, on average, 380 cells for the $w^{R N A i}$ condition and 243 cells for the $a c t n^{R N A i}$ condition. The junctional stress tensor was then renormalized (and made dimensionless) by dividing it by its own trace. Finally, the junctional stress anisotropy along the m-l axis was determined by taking the yy component of the traceless part of the normalized stress tensor. Analyses were performed in wt, $w^{R N A i}$ and $\operatorname{actn}^{R N A i}$ tissues.

Scutellum adult shape: Upon hatching, adult flies were aged for 3 days at $25^{\circ} \mathrm{C}$. They were then preserved in Glycerol:Ethanol (3:1). Animals were mounted in Glycerol:Ethanol (3:1) and imaged with a Zeiss SteREO Discovery V20, using a PlanApo S 1.0x FWD 60mm objective and Axio CAM ICc 1 camera. Scutellum elongation was determined as the ratio between maximum m-1 length of the scutellum length (v) and the a-p length of the scutellum (h, Fig S3H-H' '). The elongation measurements were performed in males and females yielding similar results (not shown). Analyses were performed in $p n r-G 4>w^{d s R N A}$ and $p n r-$ $G 4>a c t n^{d s R N A}$ animals.

\section{Hippo/Yki component localization and signaling.}

Apical-Basal Localization. To determine the apical-basal localization of Hippo pathway components at 26 hAPF, pupae expressing Jub:GFP or Warts:GFP or $\alpha$ Catenin:GFP ( $\alpha$ Cat:GFP) and E-Cadh:3xmKate2 were imaged by acquiring xy-z stacks $(0.2 \mu \mathrm{m}$ z-step). Upon acquisition, an apical-basal section of the AJ was obtained using the reslice Fiji function (slice count:3). The resulting apical-basal stacks were SUM projected. The apical-basal section was then used to determine the position of the maximal ECadh:3xmKate2 signal that could be used to define AJ's apical-basal position. Background signal of SUM projection was removed by using Fiji Subtract Background (rolling:1, sliding: disable), and resulting images were binarized by using Fiji Threshold Minimum algorithm. E-Cadh particles were extracted from binary images using Fiji Analyze Particles. SUM projected images of the GFP-protein channel were also obtained in parallel. Finally, E-Cadh AJ-corresponding particles were manually localized, and their centroids were used to estimate their z-position relative to the closest GFP-protein channel particle centroid's z-position. Mean protein z-position were reset according to E-Cadh:3xmKate2 and E-Cadh:3xGFP mean differences.

Protein distributions around the aSF tips along the AJ. To determine the distribution of PH:GFP, Actn:GFP, Jub:GFP, or Wts:GFP along the AJ centered at the tips of the aSFs, tissues expressing PH:GFP, Actn:GFP, Jub:GFP or Wts:GFP and MyoII:3xmKate 2 were imaged and average-projected ( $7 \mathrm{z}$-slices, $3 \mu \mathrm{m})$ at the AJ level. Using the Fiji Segmented Tool, the intensity profile of PH:GFP, Actn:GFP Jub:GFP or Wts:GFP along the AJ was determined, and the positions of aSF tips (marked by MyoII:3xmKate2) were annotated. The background intensity was measured in the medial apical region of the cells. Based on the positions of the annotated aSF tips, each intensity profile was registered and then averaged to obtain the PH:GFP, 
Actn:GFP, Jub:GFP or Wts:GFP distribution along the AJ about the aSF tips' positions. Similar results were obtained for Wts:GFP or Wts:CitFP (not shown). In order to visualize the curve for each protein on the same plot (Fig. S5A), from $1.5 \mu \mathrm{m}$ on one side of the aSF tip to $1.5 \mu \mathrm{m}$ on the other side of the tip, the median of each protein's (averaged) curve was subtracted from the curve itself; then, each protein's curve was normalized by the maximum value of all the distributions (in this case, Wts:GFP). Finally, to set median values around one, one was added to all resulting values. These transformations are only used for data representation and do not affect any subsequent quantitative conclusions. Percentages shown on the right side of the plots, represent the enrichment of each protein's signal at the AJ near the aSF tip $(0.54 \mu \mathrm{m}$ around the aSF tip) relative to the protein signal at the AJ away from the aSF tip (farther than $0.54 \mu \mathrm{m}$ but closer than $1.5 \mu \mathrm{m}$ from the aSF tip).

Cortical intensity measurements. Jub:GFP or Wts:GFP image stacks, containing $w^{R N A i}$ control or $a c t n^{R N A i}$ clones, were average-projected about the level of the AJs ( 7 z-slices, $3 \mu \mathrm{m})$. The integrated intensity of Jub:GFP or Wts:GFP was measured by delineating cell contours via the Fiji segmented line tool (with a line width of 10 pixels, approximatively equal to $1.1 \mu \mathrm{m})$. The background intensity in each cell was measured at the cell apex. After background subtraction, the average intensity of Jub:GFP or Wts:GFP was normalized by the average intensity of the corresponding protein in surrounding cells (excluding cells at the boundary of the clone).

Jub and Hippo Pathway component colocalization. Upon imaging of pupal tissue expressing Jub:mKate2 (or Jub:GFP) and Wts:GFP, single cells were cropped and average-projected (11 z-slices corresponding to $2 \mu \mathrm{m})$. The Jub signal was then binarized using the Otsu algorithm in Fiji to create a mask used for calculating the Pearson correlation coefficient between the Jub signal and either Jub itself or Wts signal. Analyses were performed in $a c t n^{R N A i}$ mutant cells and $w^{R N A i}$ control cells.

Yorkie transcriptional reporter level measurements. The levels of transcriptional activation of the Hippo/Yki pathway were measured using two reporters: ban-nls:GFP (23) and dIAPl-nls:GFP (55). In a given mutant clone (marked by the expression of Caax:tBFP), these levels were defined as the ratio between the nls:GFP signal inside and outside of the clone. The ratio was measured as follows: upon projection of the $n l s: G F P$ nuclear signal (see above), each $n l s: G F P$ nuclear signal was manually segmented using the ROI selection function of Fiji. The inside and outside $n l s: G F P$ signals were defined as the average $n l s: G F P$ signal within a layer of two nuclei along the clone boundary inside and outside of the clone. The background fluorescence signal was measured by averaging the GFP fluorescence signal in 5 regions located between nls:GFP marked nuclei. The level of transcriptional activation of Hippo/Yki pathway was normalized to that of control $w^{R N A i}$ clones at $18 \mathrm{hAPF}$. Similar measurements were performed for acth $^{I G I}$ mutant clones marked by the absence of nls:ChFP. To compare the level of transcriptional activation of the Hippo/Yki pathway at 18 and $26 \mathrm{hAPF}$ without possible confounding effects due to protein perdurance, the ban$n l s: G F P$ signal measurements were performed $72 \mathrm{~h} \pm 4 \mathrm{~h}$ after clone induction in both cases.

Quantification of nuclear GFP signal as a function of cell size or Jub ratio ${ }^{\text {in/out }}$ of cluster. To quantify bannls:GFP, dIAP1-nls:GFP or ubi-nls:GFP signals as a function of cell apical area, a maximum E:Cad:3xmKate2 signal projection and the average-projection of the nls:GFP signal were obtained (see above). The E-Cad:3xmKate2 and the nls:GFP signals were then segmented (see above). Each nls:GFP signal was associated with a given E-Cad:3xmKate2 segmented cell using the cKDTree tool of the package scipy.spatial in Python. The associations were manually validated and corrected, if necessary. To exclude non-epithelial cells, the nls:GFP segmented data were filtered by excluding nls:GFP nuclei whose signals are lower or higher than the mean nls:GFP nucleus intensity \pm 3 standard deviations. To facilitate the visualization of either ban-nls:GFP and ubi-nls:GFP or dIAP1-nls:GFP and ubi-nls:GFP on the same graph, by shifting one of the curves in each pair vertically (i.e., along the y-axis), the values of paired curves 
were set to be similar for the lowest cell size bin. Since this vertical shift is uniform across all size bins, it does not affect our quantitative conclusions, which only depend on the slopes of the curves. In a similar way, apical Jub:mKate2 maximum and average projections were used to determine the cell apical area and Jub ratio ${ }^{\text {in/out }}$ of cluster, respectively.

\section{MyoII level measurements.}

Junctional cortex thickness. We estimated the junctional cortex thickness around 2 min prior to the nucleation of an aSF at a given TCJ using the E-Cad:3xmKate2 and MyoII:3xGFP time-lapse movies. Upon projection of the time-lapse movies, E-Cad:3xmKate2 and MyoII:3xGFP intensity profiles were measured along a line that is 5 pixels wide $(0.13 \mu \mathrm{m}$ per pixel $)$ and passing orthogonal to the prospective aSF through the TCJ. All line profiles were then re-aligned using the position of the TCJ. Once re-aligned, intensity profiles of $0.5 \mu \mathrm{m}$ before the TCJ (on the side opposite the prospective aSF) and $2.0 \mu \mathrm{m}$ after the TCJ were considered and normalized between 0 (minimum value) and 1 (maximum values). The junctional cortex thickness was estimated by two different approaches. First, we performed a cross-correlation analysis to determine the distance between the position of E-Cad:3xmKate2 maximal signal intensity and the position of MyoII:3xGFP maximal signal intensity; this distance corresponds to half of the estimated cortex thickness. Alternatively, we estimated the junctional cortex thickness by determining the distance between the position of the MyoII:3xGFP peak and the position corresponding to 2/3 of the MyoII:3xGFP maximal intensity value. Both measurements give similar junctional cortex thickness values: $0.52 \mu \mathrm{m}$ versus $0.51 \mu \mathrm{m}$ $\pm 0.12(\mathrm{n}=90)$ (see also Fig. S8S).

Cortical Myo-II levels. Containing $w^{R N A i}$ control and $a c t n^{R N A i}$ clones, image stacks of MyoII:3xmKate2 and Jub:GFP at 18 and 26 hAPF were average-projected about the level of the AJs ( $7 \mathrm{z}$-slices, $3 \mu \mathrm{m}$ ). The average MyoII:3xmKate2 intensity for each cell was measured by delineating the cell contour via the Fiji segmented line tool (with a line width of 10 pixels, approximatively equal to $1.1 \mu \mathrm{m}$ ). MyoII:3xmKate2 background intensity in each cell was measured within a region of cell apex devoid of aSFs. After background subtraction, the MyoII:3xmKate2 average intensity was normalized by the average intensities of surrounding cells (excluding cells at the boundary of the clone). Quantification were performed in regions where MyoII level is homogenous in control cells to avoid any bias.

Pool of MyoII in elongated and ortho-elongated cells. Image stacks of MyoII:3xGFP and E-Cad:3xmKate2 at $26 \mathrm{hAPF}$ were average-projected about the level of the AJs ( $7 \mathrm{z}$-slices, $3 \mu \mathrm{m})$. Contours of individual cells were delineated by using Fiji polygon tool, and MyoII:3xGFP integrated intensities along the cell contours were obtained. The fluorescent background intensity was estimated by using the same cell contour ROI in the most apical z-slice of the stack. After background subtraction for both ortho-elongated cells and elongated cells, integrated MyoII:3xGFP intensities of ortho-elongated cells were normalized by the average integrated intensities of the elongated cells within the same confocal stacks.

\section{Statistics}

Sample sizes vary in each experiment. The experiments were repeated, at least, three independent times, except for the data shown in Fig. S3F (which was done once) and Fig. S3E, Fig 4A-B and Fig. S6A (which were repeated twice). The number of aSFs, ablations, animals or cells used in each experiment are indicated in figures or figure legends. For all graphs, each error bar represents the standard error of the mean (SEM); the only exceptions are Fig. S5D, Fig. 1D and Fig. S1S in which the standard deviation (STD) was used. The statistical tests used to assess significance are stated in the figure legends. Statistical significance between sample distributions were typically analyzed by Nonparametric ANOVA (Kruskal-Wallis test) with Conover post hoc tests (using a step-down method using Bonferroni adjustments). Mixed-ANOVA and one-tailed Wilcoxon signed rank tests were used for cell division evolution and cell division dependency 
on cell apical area. The Nonparametric Levene Test was used to analyze aSF orientations for equality of variances. Alternatively, the ANCOVA test, which combines ANOVA and regression, was used to test differences in regression slopes and intercepts between different conditions. Statistical differences are indicated by one asterisk, and $p$-values are indicated in the corresponding figure legends. In the case of

multiple pairwise comparisons, only the maximum $p$-values (of the set of asterisked comparisons) are reported in the figure legend. Statistical analyses were performed using scipy.stats, scikit_posthocs and uncertainties statistical packages $(53,56,57)$ as well as the MATLAB 2016B Statistics and Machine Learning Toolbox, MathWorks $(58,59)$.

\section{Code availability}

The MATLAB codes used to segment cells, to track cells and to quantify tissue scale dynamics have been previously published $(4,10)$. The code was modified to perform quantification on a defined region of the tissue and to extract cell division rate. The MATLAB codes, the Python Jupyter Notebook for plotting and statistical analyses, and the Fiji macros used for cluster numbers, co-localization, nuclear signal projection, as well as projections of aSFs and AJs are available upon request. 


\section{Supplementary Text}

In this supplementary text, we describe biophysical models that we have developed to understand the behavior of apical stress fibers (aSFs) in epithelia and some experimental tests of the models' predictions. Section 1 introduces a model of how aSFs affect the force balance in epithelial tissues under uniaxial stress and thereby help cells to resist elongation. In Section $1 \mathrm{C}$, we use this model to predict that for large cells to resist elongation as effectively as small cells, the total aSF tension per cell (proportional to the number of aSFs) must increase with apical area (Fig. 2I). We experimentally confirmed this increase of aSF number with apical area in wild-type tissues (Fig. 5D).

Based on the results of our time-lapse imaging (Movie S2 and Movie S11), in Section 2 we develop a geometrical model of aSF nucleation and breakage. This geometrical model, based on the role of tricellular junctions (TCJs) in aSF nucleation and breakage, explains approximately 75 percent of the observed increase of aSF number with cell apical area at $26 \mathrm{~h}$ APF. In Section 3, we test this geometrical model by generating cells of similar apical area but with different numbers of TCJs; we find that, as the model predicts, a dependence of aSF number on the number of TCJs, not just on cell apical area, is required to explain the data. We also show that our model is consistent with observations on cells of a peculiar geometry: those elongated orthogonal to the tensile uniaxial stress. In Section 4, we discuss biophysical hypotheses for the mechanisms leading to aSF nucleation primarily at TCJs with a certain orientation.

\section{Mechanical model for the role of aSFs in resisting cell elongation}

In this section, we develop a simple model to understand how the presence of aSFs affects force balance, and thus cell elongation, in epithelia. Specifically, we would like to answer two questions. First, if we fix the total amount of MyoII per cell, what is gained by shifting some of this contractile activity from AJs to aSFs? Our model predicts that compared to tension on AJs, tension on aSFs is always more effective at resisting cell elongation; this prediction is validated experimentally in Fig. S3P. The relative effectiveness of aSFs in resisting cell elongation is quite large for certain cell orientations (Fig. S4F-H). Second, how much total aSF tension per cell is required to maintain a regular cell shape under uniaxial tension, and how does the required aSF tension depend on apical cell area? Our model finds that the total aSF tension per cell should scale linearly with apical area; this prediction is tested in Figs. 2J and S3P. Thus, our model, even though quite simplified, makes meaningful and testable predictions about the role of aSFs in resisting elongation of cells under uniaxial stress.

\section{A. Vertex model with tensile aSFs}

To gain a physical understanding of the implications of adding aSFs to epithelial cells under uniaxial mechanical stress, we build on an existing 2D mechanical model of epithelial tissues. The mechanical model that we modify, called a vertex model (60-62), has been successfully used to describe a diverse set of developmental processes in Drosophila and other model organisms (61-67). In vertex models, the epithelium is represented as a tiling of the plane by polygonal cells; each polygon edge has a contractile tension, which provides resistance against external mechanical stresses. Here, to model the presence of aSFs, we incorporate additional contractile structures that span the cell and are aligned with the axis of imposed high tensile stress (Fig. 2G and Fig. S4A,B).

Specifically, we focus on the simplest case of a regular packing of identical, hexagonal cells. Similarly simplified geometries have provided useful insights into cell-cell mechanical interactions in past studies $(48,68,69)$, and in our case this model's analytic tractability will allow us to extract a prediction for the aSF tension per cell necessary to resist elongation as a function of apical area. Importantly, we assume that, because aSFs are mobile, they can align with other aSFs or with contractile junctions in neighboring 
cells (Fig. S4A,B). This mobility, together with the fact that aSFs can always align with the high stress axis (unlike junctions, which must enclose the cell and thus cannot all be aligned), is central to aSFs' superiority to junctional tension in limiting cell elongation. It is easy to verify that, if all aSFs are aligned with an appropriate structure in adjacent cells, the same results are obtained whether the aSF tension is all carried by one aSF or is split among multiple aSFs per cell (Fig. 2G). Our calculations are thus carried out in terms of the total aSF tension $\gamma_{f}$ per cell. Depending on the context, we can phrase our results either in terms of $\gamma_{f}$ or in terms of the number of aSFs per cell with some fixed tension per aSF (with the latter language, as discussed below, probably a better description of what actually occurs in the dorsal thorax).

With these assumptions, our tissue geometry is specified by two edge lengths and the angle of one edge with the horizontal (Fig. 2G and Fig. S4A,B). In general, the cell packing's response to an imposed uniaxial tension depends on the cells' orientation with respect to the axis of high stress (69). We perform our calculations for the two distinct limiting cases of this orientation, which we term the 'cable-forming orientation' (CFO) and the 'non-cable-forming orientation' (NCFO) (Fig. S4A,B). Cells in the CFO become brick-shaped under tensile stress, which favors the formation of supracellular actomyosin cables. Cells in the NCFO elongate under tensile stress and do not form supracellular actomyosin cables. (For a detailed discussion of the response of the CFO and NCFO to uniaxial stress, see (69).) For each orientation we can describe the tissue in terms of six variables: two edge lengths $l_{i}$, an edge angle $\theta$ or $\phi$, two edge tensions $\gamma_{i}$, and the total aSF tension $\gamma_{f}$.

By ablation of aSFs, we estimate that the tension per aSF is roughly independent of cell apical area (Fig. S3L); therefore, we conclude that cell-to-cell variability in total aSF tension depends primarily on differences in aSF number rather than on differences in individual aSF tensions. However, as discussed above, in the model we keep track only of the total aSF tension $\gamma_{f}$, and we view multiple aSFs in the same cell as behaving the same as a single, lumped aSF with the corresponding $\gamma_{f}$. Although our model simplifies the dynamics and organization of aSFs, it will provide a testable prediction for the relationship between cell apical area and the number of aSFs per cell required for cells to remain regular under uniaxial stress.

\section{B. Mathematical formulation}

There are four physical constraints relating the six variables that describe each cell (Fig. S4A,B). We assume that the cells are incompressible with a constant, given apical area $A$ :
$A=2 l_{1} \sin (\theta)\left(l_{2}+l_{1} \cos (\theta)\right)$
(CFO);
[Eq. 1]
$A=2 l_{4} \cos (\phi)\left(l_{3}+l_{4} \sin (\phi)\right)$
(NCFO).

For the tissue to be in mechanical equilibrium, tension forces at each vertex must balance:
$\cos (\theta)=\frac{\gamma_{2}}{2 \gamma_{1}}$
(CFO);
[Eq. 3]
$\sin (\phi)=\frac{\gamma_{3}-\gamma_{f}}{2 \gamma_{4}}$
(NCFO).
[Eq. 4]

(Please note that because all cells are identical, any pressures conjugate to cell area drop out of the force balance equations and can be neglected.) We assume that the tension on each edge and aSF is proportional to the concentration of Myosin II (MyoII) on that edge and that the total amount $\lambda$ of MyoII per cell is fixed but may be redistributed from one edge or aSF to another. This assumption is certainly an oversimplification but reflects the essential fact that cell resources are limited and thus infinite reservoirs of MyoII are not available to counteract imposed mechanical stresses. This assumption implies the constraint: 
$\lambda=2 \gamma_{1} l_{1}+\gamma_{2} l_{2}+\gamma_{f}\left(2 l_{1} \sin (\theta)\right)$

$\lambda=2 \gamma_{4} l_{4}+\gamma_{3} l_{3}+\gamma_{f}\left(l_{3}+2 l_{4} \sin (\phi)\right)$

Finally, the difference $\sigma_{D}=$
the tissue is viewed as a fixed
$\sigma_{D}=\frac{\gamma_{1} \sin (\theta)+\gamma_{f}}{l_{2}+l_{1} \cos (\theta)}-\frac{\gamma_{2}}{2 l_{1} \sin (\theta)}$

$\sigma_{D}=\frac{\gamma_{3}+\gamma_{f}}{2 l_{4} \cos (\phi)}-\frac{\gamma_{4} \cos (\phi)}{l_{3}+l_{4} \sin (\phi)}$

Given these four constraints, we have two free parameters in the model, which we generally take to be the aSF tension and one of the two edge tensions.

We are interested in the relation between cell elongation and aSF number. Our measure of elongation is $S=P / \sqrt{A}$, where $P$ is the cell perimeter (70-72). In this packing of identical, hexagonal cells, larger values of $S$ imply that a polygon is more elongated. $S$ is a mathematically convenient measure of cell elongation because $S$ has a physically relevant global minimum when the hexagons are neither elongated along the uniaxial stress nor elongated orthogonal to this stress. If we had chosen a measure that tracks elongation specifically along the uniaxial stress, minimizing that measure of cell elongation would maximally elongate cells orthogonal to the uniaxial stress, generating a singular and physically irrelevant cell geometry. For cells in the CFO,

$S\left(\gamma_{2}, \gamma_{f} ; \sigma_{D}, A, \lambda\right)=\frac{\lambda-A \sigma_{D}}{\sqrt{A} \gamma_{2}}+\frac{\lambda+A \sigma_{D}}{\sqrt{A}\left(\tan (\theta)+2 \gamma_{f}\right)}\left(\frac{2-\cos (\theta)}{\sin (\theta)}\right)$, where $\tan (\theta)=\frac{\lambda^{2}-A^{2} \sigma_{D}{ }^{2}}{2 \gamma_{2}{ }^{2} A}-2 \frac{\gamma_{f}}{\gamma_{2}}$. [Eq. 9]

For cells in the NCFO,

$S\left(\gamma_{3}, \gamma_{f} ; \sigma_{D}, A, \lambda\right)=\frac{\lambda+A \sigma_{D}}{\sqrt{A}\left(\gamma_{3}+\gamma_{f}\right)}+\frac{\left(\lambda-A \sigma_{D}\right) \tan (\phi)}{\sqrt{A}\left(\gamma_{3}-\gamma_{f}\right)}\left(\frac{2-\sin (\phi)}{\cos (\phi)}\right)$, where $\tan (\phi)=\frac{2 A\left(\gamma_{3}{ }^{2}-\gamma_{f}{ }^{2}\right)}{\lambda^{2}-A^{2} \sigma_{D}{ }^{2}}$. [Eq. 10]

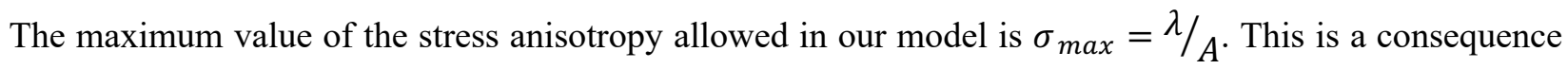
of having restricted both the apical cell area and the total amount of MyoII, which effectively limits the tension per unit length that can be obtained in the system.

\section{C. Mechanical model predicts increase of aSF number with apical area}

At a fixed apical area, we determine the minimal cell elongation as a function of the tissue stress anisotropy and total aSF tension per cell by minimizing $S\left(\gamma_{i}, \gamma_{f} ; \sigma_{D}, A, \lambda\right)$ with respect to the value of the tension on the high-tension edge $\gamma_{i}$. We show the results of this minimization in Fig. S4D-H, where we have normalized the cell elongation by the elongation of a regular hexagon $\left(S_{\text {reg,hex }}=\frac{P}{\sqrt{A}}=6 / \sqrt{\frac{3 \sqrt{3}}{2}}\right)$ and the stress anisotropy $\sigma_{D}$ by $\sigma_{\max }$; note that the results expressed in this nondimensionalized fashion are independent of $A$ and $\lambda$. Under uniaxial stress, if cells have no aSFs, it is not possible for cells to remain regular hexagons (Fig. S4F). In both the CFO and the NCFO, we find that the presence of aSFs reduces elongation at fixed total MyoII $\lambda$; that is, aSFs are more effective in resisting cell elongation than simply upregulating tension on cell-cell junctions. For minimizing cell elongation, cells in the NCFO benefit more from redistributing junctional MyoII to aSFs than cells in the CFO do (Fig. S4F). 
In both orientations and for any allowable stress anisotropy, there exists some distribution of MyoII between the edges and aSFs such that the cell is a regular hexagon (i.e., normalized elongation parameter equal to one). At minimal cell elongation, we can exploit the following geometric constraints:
$l_{1}=l_{2}$ and $\theta=\pi / 3$
(CFO);
[Eq. 11]
$l_{3}=l_{4}$ and $\phi=\pi / 6$
(NCFO).
[Eq. 12]

to find an analytic solution for the distribution of MyoII between the edges and the aSFs. In the NCFO,

$$
\begin{aligned}
& \gamma_{3}=\sqrt{\frac{\sqrt{3}}{6}} \frac{\lambda}{\sqrt{A}} ; \\
& \gamma_{f N C F O}=\sqrt{\frac{\sqrt{3}}{6}} \sqrt{A} \sigma_{D} ; \\
& \gamma_{4}=\gamma_{3}-\gamma_{f N C F O} .
\end{aligned}
$$

In the CFO,

$$
\begin{aligned}
& \gamma_{1}=\gamma_{2}=\sqrt{\frac{\sqrt{3}}{6}}\left(\frac{\lambda-A \sigma_{D}}{\sqrt{A}}\right) ; \\
& \gamma_{f C F O}=\sqrt{\frac{\sqrt{3}}{2}} \sqrt{A} \sigma_{D} .
\end{aligned}
$$

In both orientations (Eq. 14 and Eq. 17), for the cells to remain regular hexagons under uniaxial stress, the total aSF tension per cell must be proportional to $\sqrt{A} \sigma_{D}$. Since in the dorsal thorax the tension per aSF does not depend on apical area (Fig. S3L), large cells need more aSFs per cell than small cells to resist elongation under the same stress anisotropy. This finding is reminiscent of the fact that a dry foam of large bubbles is less resistant to extensional shear than a dry foam of small bubbles $(73,74)$. Since twodimensional stress is a measure of force per length (Fig. S4C), to remain regular hexagons, larger cells must compensate for the greater distance between edges by increasing the number of aSFs.

In summary, our mechanical model provides three predictions. First, at constant apical area and stress, cells with no aSFs will be less elongated in the CFO than in the NCFO (69). Second, at constant apical area, stress, and orientation, cells with more aSFs are less elongated along the axis of high tensile stress than cells with fewer aSFs. Lastly, at constant stress and minimal cell elongation, larger cells will have more aSFs. Since the tissue of interest (Fig. 1C) is disordered, it is difficult to apply these predictions directly to experimental data. We do, however, test a broad claim of the model: for cells to resist elongation due to tensile stress, the number of aSFs per cell must increase linearly with the square root of the apical area (Fig. S4I). Over the range of observed wild-type cell sizes, the square root of the apical area is linear in the area to a very good approximation (Fig. S4J), so we use cell apical area, the more biologically intuitive measure of cell size, to test this model claim.

We confirm in Fig. 5D that the number of aSFs per cell linearly increases with apical area at $26 \mathrm{~h}$ APF. The 95\% confidence interval for the linear regression coefficient is $\left(0.058 \frac{a S F}{\mu m^{2}}, 0.061 \frac{a S F}{\mu m^{2}}\right)$. Via an $F$-test $(58,59)$, we find that the regression coefficient is significantly different than zero $\left(p<10^{-50}\right)$. Based on our vertex model with aSFs (Eq. 14 and Eq. 17), we hypothesize that the wild-type linear increase of aSF number with cell apical area is necessary for large cells to resist cell elongation as effectively as small cells; in the main text, we test this hypothesis by showing that cell elongation is independent of apical area in 
control $w^{R N A i}$ cells at $26 \mathrm{~h}$ APF (Fig. S3P). Then, as predicted by our mechanical model, we show that when the scaling of aSF number with apical area is much weaker than the control $w^{R N A i}$ scaling as observed in $a c t n^{R N A i}$ cells (Fig. 2J), larger cells elongate more than smaller cells (Fig. 2M and Fig. S3P).

\section{Geometrical model of aSF nucleation and breakage}

In this section we use time-lapse imaging of cells in the tissue of interest (Movie S2 and Movie S11) to develop a phenomenological model of how cell geometry affects the number of aSFs in individual cells. In these time-lapse images, MyoII:3xGFP marks the junctional cortex and aSFs, and E-Cad:3xmKate2 marks the adherens junctions (AJs). We find that aSFs nucleate from TCJs in an orientationally dependent manner: if a TCJ's bisecting vector in a cell points mostly along the anterior-posterior axis, aSFs tend to nucleate from that TCJ into that cell (Fig. 5A,B, S7A-G). Based on this aSF nucleation criterion, we first construct a model in which the aSF nucleation rate in a cell is proportional to the number of aSF-nucleating TCJs and in which all aSFs have the same mean lifetime (Section 2B). As observed in time-lapse imaging (Fig. S8O), this model predicts that larger cells have higher aSF nucleation rates per cell. However, the model shows that nucleation effects alone only account for approximately 15 percent of the wild-type scaling of aSF number with apical area (Fig. S7J).

Since a model of aSF nucleation rates alone cannot adequately explain the wild-type scaling of aSF number with cell apical area, we analyze how aSF breakage rates depend on cell apical area. We find that aSFs in larger cells have longer lifetimes, on average, than aSFs in smaller cells (Fig. S8P). To build a coarse-grained model of aSF breakage, we track aSFs from nucleation to breakage. We find that aSFs tend to break at TCJs (Fig. 5A,C, S8E,F,J). For the TCJs at which aSFs break, the bisecting vectors within the cell of interest point mostly along the medial-lateral axis (Fig. 5A, S7A,B, S8E,F, L,M). Based on these observations of aSF nucleation and breakage at TCJs, we construct a phenomenological model in which aSFs move at a constant speed along the anterior-posterior axis from an aSF-nucleating site to aSF-breaking sites. We experimentally constrain all but one of the model parameters. The one free parameter is the average signed distance between the leading aSF tip and breaking TCJ at the time of breakage (see Fig. S8T and Section 2D). This cell-autonomous model, with one free parameter, explains approximately 75 percent of the experimentally observed wild-type scaling of aSF number with apical area (Fig. 5D); encouragingly, the best-fit value of the one free parameter is on the order of the junctional cortex thickness and is, thus, amenable to a reasonable physical interpretation (Fig. S8T).

It is worth emphasizing that all the models in this section are intended to describe cells in tissues where aSF nucleation occurs at an appreciable rate. In Drosophila pupal dorsal thorax, such nucleation appears to happen only when the tissue is under substantial uniaxial mechanical tension. Thus, we do not suggest that all TCJs, in any tissue, obeying our geometric criteria can nucleate aSFs. Rather, our models claim that TCJs with an appropriate bisector orientation and in a tissue with the requisite mechanical stress will tend to serve as nucleating TCJs. In this section, we do not delve into the question of why mechanical stress together with TCJ orientation favor aSF nucleation, but we discuss some hypotheses and speculations on this point in Section 4.

\section{A. aSF nucleation at TCJS}

One mechanism by which aSF number in a cell could be controlled by cell geometry is if cells produced aSFs at different rates based on apical area. More specifically, if certain sites act as sites of aSF nucleation, aSF number per cell could be controlled by changing the number of nucleating sites. In timelapse images of individual cells (Movie S2 and Movie S11), we find that aSFs nucleate from the cell cortex near TCJs. Over more than 600 nucleation events, we find that more than 95 percent of aSFs nucleate from a TCJ (Fig. 5C). Less than 5 percent of the nucleation events are due to apoptosis or division of a neighboring 
cell (Fig. 5C). In the cases of aSF nucleation during division of a neighboring cell, the cell-cell junction shared with the dividing cell becomes curved, and the aSF nucleates from the curved region of the cell-cell junction. Indeed, this curved region will become a TCJ after cytokinesis is completed.

Although the association of other force-generating structures with TCJs is well-documented $(3,8$, $26,48,75-77)$, to the best of our knowledge, we are the first to describe TCJs as sites of SF nucleation. Contrary to many other examples of SFs, which are mostly stationary $(25,78-85)$, these aSFs, that nucleate at a TCJ, move along cell-cell junctions towards the center of the cell (Movie S2 and Movie S11). Before discussing the dynamics of aSFs after nucleation, in Section $2 \mathrm{C}$, we would like to know if specific geometric properties of TCJs determine from which TCJs aSFs nucleate and from which TCJs aSFs do not nucleate.

In this study, to characterize TCJ geometry as a predictor of aSF nucleation, we measure two angles for each pair of adjacent edges that meet at the TCJ: the opening angle, $\alpha$, between the adjacent edges and the orientation of the bisecting vector, $\theta$, relative to the anterior-posterior axis (Fig. S7A). We calculate $\alpha$ and $\theta$ immediately prior to nucleation for more than 600 nucleation events. We exclude nucleation events due to mitosis or apoptosis of a neighboring cell.

In a mechanically isotropic tissue, rotational symmetry must be respected, so nucleation rates per TCJ must only depend on differences between edge orientations (i.e., opening angle between two edges). Because the dorsal thorax is under anisotropic stress, we expect that the nucleation rate is not simply a function of opening angle but likely also has some $\theta$ dependence. Additionally, we expect the results to be invariant under $\theta \rightarrow-\theta$ and $\rightarrow \pi-\theta$. For this reason, we define $\theta$ as $\left|\tan ^{-1}\left(\frac{\vec{\mu} \cdot \hat{y}}{\vec{\mu} \cdot \hat{x}}\right)\right|$, where $\vec{\mu}$ is the bisecting vector of the TCJ. The anterior-posterior (a-p) axis corresponds to $\theta=0$; the axis of medial-lateral (m-l) stress corresponds to $\theta=\frac{\pi}{2}$. Given this definition, we compare the angles $\alpha$ and $\theta$ for nucleation events to the angles $\alpha$ and $\theta$ of all pairs of adjacent edges, meeting at a TCJ, from still images of the same region of the Drosophila dorsal thorax at the same time in development. We quantify whether aSF nucleation events are restricted to certain regions of this two-dimensional space, $\alpha$ and $\theta$.

In Fig. S7C, we compute the fraction of pairs of adjacent edges, which meet at a TCJ, with angles $\alpha$ and $\theta$ in the region of interest in approximately 25,000 cells at $26 \mathrm{~h}$ APF. As expected, there is a correlation between $\alpha$ and $\theta$ in these tissues under anisotropic stress because cells tend to elongate along the axis of stress $(69,86)$. Fig. S7D shows the fraction of nucleation events as a function of $\alpha$ and $\theta$. In Fig. S7E, we show the nucleation rate from pairs of adjacent edges of given $\alpha$ and $\theta$ (i.e., the ratio of data from Fig. S7D to data from Fig. S7C). In Fig. S7E, we exclude, indicated by hatching, values of $\alpha$ and $\theta$ which are very rarely observed (i.e., value of less than $0.01 \%$ in Fig. S7C).

The nucleation rates from Fig. S7E appear to be relatively insensitive to opening angle $\alpha$ but strongly dependent on bisector orientation $\theta$ relative to the a-p axis. To test the sensitivity of nucleation rate to these two angles, we linearly regress the nucleation rates from Fig. S7E on both $\alpha$ and $\theta$. Via a $t$-test for the $\alpha$ regression coefficient in this bivariate regression $(58,59)$, we find that the nucleation rate per TCJ is insensitive to $\alpha(p=0.07)$. Via a $t$-test for the $\theta$ regression coefficient $(58,59)$, we find that nucleation rate per TCJ is sensitive to $\theta$ (i.e., the corresponding coefficient in the regression is non-zero; $p<10^{-15}$ ). For the remaining analysis, we reduce this problem to one dimension, tracking only $\theta$ for each aSF nucleation event.

In Fig. S7F, we plot the empirical cumulative distribution of the aSF nucleation data versus $\theta$ (i.e., the fraction of aSF nucleation events at TCJs with $\theta^{\prime}<\theta$ ). Given an image of a cell in the dorsal thorax, based on the $\theta$ value of each pair of adjacent edges, we want to predict which TCJs nucleate aSFs and which 
do not. For the sake of simplicity, we caricature the observed $\theta$-dependence of aSF nucleation as a cutoff, which we calculate based on the $95^{\text {th }}$ percentile of aSF nucleation events shown in Fig. S7F. This calculation

returns a cutoff for aSF nucleation at $\theta \approx \frac{2}{3}$. After reducing the problem of aSF nucleation in epithelia to a one-dimensional cutoff based on TCJ bisector orientation $\theta$, we ask whether and how strongly the number of aSF-nucleating TCJs depends on cell apical area.

\section{B. Large cells have more nucleating TCJs than small cells}

To reach a steady state in which large cells have more aSFs than small cells, large cells could have higher aSF nucleation rates and/or lower aSF breakage rate. Below, we will explore how the tendency of aSF to break at TCJs implies that breakage events are less frequent in larger cells. In this section, however, we first calculate what percentage of the scaling of aSF number with cell apical area can be explained by differences in the number of aSF-nucleating sites alone.

Based on the findings of Section 2A, we estimate the rate of aSF nucleation for each cell by counting the number of TCJs that satisfy the aSF nucleation criterion (Fig. S7F,G,I). Encouragingly, in our tracking of aSF nucleation in time-lapse imaging, we find that aSF nucleation rate per cell $i$ proportional to the number of nucleating TCJs (Fig. S7H). We assume that the rate of aSF nucleation per nucleating TCJ is independent of cell apical area. If we further assume that aSFs break at a constant rate $\gamma$ per time, independent of both cell apical area and of their distance from TCJs, the mean number of aSFs in a cell should be directly proportional to the number of nucleating TCJs in that cell. The constant of proportionality is $k_{n u c} / \gamma$, where $k_{n u c}$ is the aSF nucleation rate per nucleating TCJ.

To test this "nucleation-only" model, we use snapshots of approximately 25,000 cells, for which we count the number of nucleating TCJs and the number of aSFs, across 35 dorsal thoraces in the region boxed in Fig. 1A. We use a standard technique for determining the incremental contributions of variables to explaining variance (58). In this case, we want to know how much of the variance of aSF number is explained by cell apical area beyond the variance of aSF number explained by the number of aSF-nucleating TCJs. We find that the nucleation-only model, based on differences in aSF nucleation rates alone, explains approximately 15 percent of the observed scaling of aSF number with apical area.

We illustrate the physical content behind the method in (58) in Fig. S7J. To estimate $k_{n u c} / \gamma$, we linearly regress aSF number in each cell versus the number of nucleating TCJs, constraining the intercept to zero, as demanded by the model. This fit returns that $k_{n u c} / \gamma$ is approximately one aSF per four nucleating TCJs (95\% CI: $\left(0.28 \frac{a S F}{\text { nucleating TCJ }}, 0.29 \frac{a S F}{\text { nucleating } T C J}\right)$ ). To illustrate the scaling of aSF number with cell apical area predicted by the model, we bin the cells by apical area. In each bin, to estimate the mean aSF number predicted by the model, we calculate the mean number of nucleating TCJs multiplied by our fit parameter $k_{n u c} / \gamma$. We also calculate the experimentally observed mean number of aSFs for cells in each bin.

As illustrated in Fig. S7J, the nucleation-only model produces a scaling of aSF number with cell apical area of approximately $0.01 \frac{a S F}{\mu m^{2}}$. The observed scaling of aSF number with cell apical area is approximately $0.06 \frac{a S F}{\mu m^{2}}$. The nucleation-only model thus produces a scaling of aSF number with apical area that is approximately 15 percent of the scaling observed in wild-type tissues at $26 \mathrm{~h} \mathrm{APF} \mathrm{(Fig.} \mathrm{S7J).}$

The limited ability of this nucleation-only model to explain the scaling of aSF number with cell apical area suggests to us that aSF breakage is also influenced by cell geometry. If, in addition to aSF nucleation rates, aSF lifetime is positively correlated with cell apical area, that correlation could help to 
explain the scaling of aSF number with apical area that is observed. As shown in Fig. S8P, by tracking aSF lifetimes in time-lapse images in 51 distinct cells, we find that aSF lifetime is correlated with cell apical area. To search for a mechanism which explains this correlation between aSF lifetime and cell apical area, we track the aSF movement and aSF breakage in Section 2C, in a similar manner to our tracking of aSF nucleation. We will find that the cell apical area dependence of lifetimes can largely be explained if, as observed, aSF tend to break at appropriately oriented TCJs.

\section{C. aSF breakage at TCJs}

As shown in Fig. S8P, we find that aSF lifetime is correlated with cell apical area, which possibly explains why our model in Section 2B only accounts for about 15 percent of the scaling of aSF number with cell apical area. To search for a mechanism which could cause a correlation between aSF lifetime and cell apical area, we track positions of 624 aSFs at midlife and at breakage (Fig. 5A and Fig. S8A,B). We test if aSFs tend to break at certain sites in a cell. The existence of aSF-breaking sites could generate a correlation between cell apical area and aSF lifetime if the distance between the sites at which aSFs nucleate and the sites at which aSFs break increases with cell apical area.

In the time-lapse images (Movie S2 and Movie S11), we find two facts about aSF positions at breakage that are not mutually exclusive:

(1) aSFs break near the cell center.

(2) aSFs break with endpoints near at least one TCJ.

Before demonstrating that TCJs are sites of aSF breakage, we examine point (1). Since the aSFs are mostly aligned with the m-l axis (Fig. 1D), to quantify how far an aSF penetrates into a cell, we calculate the distance along the a-p axis between the nucleating TCJ and the leading tip of the aSF (i.e., the endpoint which penetrates farther into the cell from the nucleating TCJ) (Fig. S8B). This penetration distance is the y-coordinate of Fig. S8C-D. Along the x-axes of Fig. S8C-D, we plot the separation along the a-p axis between the cell centroid and the nucleating TCJ (Fig. S8B).

We find that if the distance between the nucleating TCJ and cell centroid is larger, the aSF penetrates farther, on average, into the cell by the time of breakage (the regression coefficient is non-zero; $F$-test, $p<$ $\left.10^{-50}\right)$ (Fig. S8D). The 95 percent confidence interval for the slope of the regression is $(0.74,0.93)$, and the 95 percent confidence interval for the y-intercept is $(-0.21 \mu \mathrm{m}, 0.32 \mu \mathrm{m})$ (Fig. S8D). We conclude that by the time of aSF breakage, aSFs penetrate, on average, about 85 percent of the distance from the nucleating TCJ to the cell centroid (Fig. S8D).

We ask whether aSFs are stationary from midlife to breakage or whether they are, on average, moving towards the cell centroid. For each aSF, we calculate the difference between how far the aSF has penetrated at midlife and at breakage; we find that aSFs move $0.69 \pm 0.03 \mu \mathrm{m}$ (mean \pm SEM) towards the cell centroid from midlife to breakage. At aSF midlife, we find that aSFs penetrate, on average, about 55 percent (as compared to 85 percent) of the distance from the nucleating TCJ to the cell centroid (Fig. S8C). (The 95 percent confidence interval for the slope of the regression is $(0.46,0.62)$, and the 95 percent confidence interval for the y-intercept is $(-0.01 \mu \mathrm{m}, 0.44 \mu \mathrm{m})$.) We conclude that aSFs do not stall for an appreciable amount of their lifetimes before breakage; this is consistent with a picture in which an aSF constantly move away from its nucleating TCJ until it reaches a breaking site (Fig. 5A, S7A, S8T and Movie S2 and Movie S11).

We now test whether TCJs are sites of aSF breakage (i.e., point (2)). Specifically, we would like to show that aSFs are not only likely to break near TCJs, but that they are more likely to do so than would be expected solely from a bias towards breaking near the cell centroid. For example, in Fig. S8E,F, each aSF 
is aligned with two TCJs, and each aSF breaks after aligning with the TCJs. However, the two TCJs with which the aSF is aligned also happen to be near the cell centroid. We need to make sure that such observations of aSF breakage at TCJs do not simply arise due to a correlation between TCJ positions and cell centroid positions.

Because the two aSF tips are on opposite sides of the cell (i.e., along the m-l axis about the nucleating TCJ position), each aSF tip encounters a different set of TCJs as the aSF moves from the nucleating TCJ to the cell centroid. We identify which aSF tip has penetrated farther into the cell from the nucleating TCJ. We, then, calculate the distance along the a-p axis between that aSF endpoint and its nearest TCJ (i.e., on the same side of the cell as the leading aSF endpoint). We store all these distances (one per aSF) in a vector called $\vec{\delta}^{a S F-T C J}$. We seek to test whether the leading aSF tip is closer to its nearest TCJ at the time of breakage than would be expected in a model in which leading aSF tip positions are simply correlated with the cell centroid position (Fig. S8D).

To test whether aSFs tend to break at TCJs and not just near the cell centroid, we develop a procedure for identifying pairs of aSFs which have similar distances from nucleating TCJ to cell centroid but different cell geometries. First, we build a vector of distances along the a-p axis between each nucleating TCJ and cell centroid (i.e., x-coordinate in Fig. S8D), which we call $\vec{x}$. We also build a vector of penetration distances (i.e., y-coordinate in Fig. S8D), which we call $\vec{y}$. In each of these vectors, there is one entry per aSF. Looping through the vector $\vec{x}$, for each aSF $j$, we find an aSF $i \neq j$ within another cell such that $\left|x_{i}-x_{j}\right|$ is minimized. Once aSF $i$ has been matched with aSF $j$, we enforce that aSF $i$ cannot be matched with any other aSFs. For an example of aSFs (in different cells) which have the same separation between nucleating TCJ and cell centroid, see Fig. S8E,F. Since this procedure depends on the order in which we loop through the list of aSFs, we repeat the procedure with random permutations of the looping order several times, generating different bijective maps.

Given a bijective map from each aSF $j$ to an accompanying aSF $i$, we want to test whether each aSF's breakage position depends on TCJ positions within a cell, not only on the position of the cell center. To test this, we swap aSF $i$ into the cell which contains aSF $j$ (i.e., a different cell with different TCJ positions) (Fig. S8G). To swap aSF $i$ into another cell, we calculate the coordinate (along the a-p axis) of the leading edge of the aSF $i$, which we call $\operatorname{pos}_{a-p}^{a S F} i$,swapped , in the cell which contains aSF $j$ :

$\operatorname{pos}_{a-p}^{a S F i, \text { swapped }}=\operatorname{pos}_{a-p}^{n u c j}+y_{i} * \operatorname{sign}\left(\operatorname{pos}_{a-p}^{a S F j}-\operatorname{pos}_{a-p}^{n u c j}\right)$.

In Eq. 18, $\operatorname{pos}_{a-p}^{n u c j}$ is the coordinate (along the a-p axis) of the TCJ at which aSF $j$ nucleated, and $\operatorname{pos}_{a-p}^{a S F} j$ is the coordinate (along the a-p axis) of the leading edge of the aSF $j$ in its original cell. We translate the one-dimensional coordinate $\operatorname{pos}_{a-p}^{a S F}$ i,swapped into a two-dimensional coordinate by computing the intersections between a line (along the m-l axis) at $\operatorname{pos}_{a-p}^{a S F} i$,swapped and the cell outline (represented as a polygon - with straight edges) (polyxpoly; MATLAB 2016B Mapping Toolbox, MathWorks, Natick, MA). The swapped leading tip of aSF $i$ is on the same side of the cell as the leading tip of aSF $j$ (i.e., we choose the intersection on that side of the cell). For example, if the leading tip of aSF $j$ is "above" (along the m-1 axis) the nucleating TCJ, the swapped leading tip of aSF $i$ is also "above" (along the $\mathrm{m}-1$ axis) the nucleating TCJ (Fig. S8G).

Now, we can compute how close this swapped aSF tip is to its nearest TCJ in the cell into which it has been swapped (Fig. S8G). This swapped aSF tip comes from a cell with a different distribution of TCJs but with the same distance from nucleating TCJ to cell centroid; therefore, as our null model, we can use the distance between the swapped aSF and its nearest TCJ in the cell into which it has been swapped. Our 
null model is that aSFs break near the cell centroid, but that their positions at breakage are otherwise uncorrelated with TCJ positions.

We store these distances (i.e., one per aSF) between each swapped aSF and its nearest TCJ in the cell into which it has been swapped in a vector called $\vec{\delta}^{a S F-T C J}$ swapped. Now, we compare $\vec{\delta}^{a S F-T C J}$ to

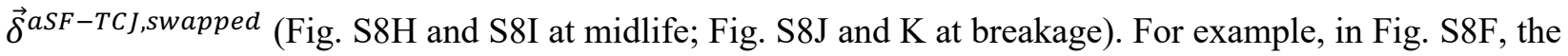
leading tip of the aSF is approximately $0.1 \mu \mathrm{m}$ from its nearest TCJ. When we swap an aSF from another cell, the leading tip of the swapped aSF is approximately $0.8 \mu \mathrm{m}$ from its nearest TCJ (Fig. S8G). Via a Mann-Whitney U-test, we compare the medians of $\vec{\delta}^{a S F-T C J}$ and $\vec{\delta}^{a S F-T C J}$ swapped at aSF breakage (Fig. $\mathrm{S} 8 \mathrm{~J}$ and $\mathrm{K}$, respectively). The two distributions have different medians $\left(p<10^{-9}\right)$. The median of $\vec{\delta}^{a S F-T C J}$ is $0.46 \mu \mathrm{m}$, and the median of $\vec{\delta}^{a S F-T C J}$ swapped is $0.67 \mu \mathrm{m}$.

We have shown that aSF leading tips tend to be near TCJs at the time of breakage, but to associate TCJs with aSF breakage, we must show that aSF leading tips are not near TCJs at midlife. We repeat the procedure outlined above, using the cell geometries at midlife (i.e., the data in Fig. S8C rather than the data in Fig. S8D). We build two vectors $\vec{\delta}^{a S F-T C J}$ and $\vec{\delta}^{a S F-T C J}$ swapped for aSF positions at midlife (Fig. S8H and S8I, respectively). We compare the medians of $\vec{\delta}^{a S F-T C J}$ and $\vec{\delta}^{a S F-T C J \text {,Swapped }}$ via a Mann-Whitney U-test, and find that their medians are not statistically significantly different $(p>0.5)$. The medians of both distributions are approximately $0.7 \mu \mathrm{m}$.

In conclusion, we have shown the following:

(1) A large majority of aSFs originate from a nucleating TCJ.

(2) aSFs move from the nucleating TCJ towards the cell center.

(3) At midlife, the leading aSF tip is not statistically significantly close to a TCJ.

(4) At breakage, the leading aSF tip is statistically significantly close to a TCJ.

We proceed to analyze geometric properties of TCJs which are close to the leading tip of the aSF at the time of breakage. Given a snapshot of a cell outline with the positions of the TCJs, we would like to predict the TCJs at which aSFs break.

We associate an aSF breakage event with a specific TCJ if, at the time of breakage, the leading aSF tip is plus or minus $0.5 \mu \mathrm{m}$ from the TCJ (i.e., approximately four pixels), along the a-p axis. Our physical justification for this $0.5 \mu \mathrm{m}$ cutoff is that the average cortex thickness is $0.5 \mu \mathrm{m}$ (Fig. S8S). If an aSF tip is less than $0.5 \mu \mathrm{m}$ from a TCJ, the aSF can link up to the cortex in the neighboring cell. Based on this criterion, a majority of the aSFs break when the leading tip of the aSF is at a TCJ. We report the geometric properties of these TCJs associated with aSF breakage in Fig. S8L,M.

In Section 2A, we computed the fraction of pairs of adjacent edges, which meet at a TCJ, with angles $\alpha$ and $\theta$ in the region of interest (Fig. S7C). We, then, calculated the fraction of nucleation events as a function of $\alpha$ and $\theta$ (Fig. S7D). We estimated the nucleation rate from pairs of adjacent edges of given $\alpha$ and $\theta$ (i.e., the ratio of data from Fig. S7D to data from Fig. S7C). We cannot repeat that procedure to estimate the propensity of aSF breakage at TCJs of given $\alpha$ and $\theta$. To estimate the propensity of breakage at TCJs with given $\alpha$ and $\theta$, we need an estimate of how many aSFs per unit time encounter TCJs with given $\alpha$ and $\theta$; then, we could compare the actual count of aSF breakage events to the number of times that aSFs reach TCJs with given $\alpha$ and $\theta$. Nonetheless, by comparing Fig. S7C to Fig. S8L, we find that aSF breakage events occur at TCJs with large bisector orientation $\theta$ and that aSF breakage at TCJs appears to be insensitive to opening angle $\alpha$. 
In the same way that we calculated a cutoff for aSF-nucleating TCJs in Fig. S7F, we plot the empirical cumulative distribution of the aSF breakage data versus $\theta$ (i.e., the fraction of aSF breakage events for TCJs with $\theta^{\prime}<\theta$ ) (Fig. S8M). We calculate a cutoff based on the $5^{\text {th }}$ percentile of aSF breakage events (Fig. S8M). The cutoff for aSF-breaking TCJs is at $\theta \approx 1$ radian. Given this cutoff, we proceed to develop a phenomenological model of aSF movement and breakage.

\section{D. Phenomenological model of aSF movement and breakage}

In Section 2C, we discussed that the correlation between aSF lifetime and cell apical area might arise because of aSF breakage at specific TCJs. We have not specified how breakage at TCJs would explain this correlation. In this section, we propose a phenomenological model for aSF movement and breakage. We will, then, employ this model to quantify how much of a correlation between aSF lifetime and cell apical area that it generates. The model of aSF movement and breakage is as follows:

(1) All TCJs that meet the aSF nucleation criterion (Fig. S7F,G) nucleate aSFs at the same rate, which we call $k_{n u c}$. aSFs do not nucleate at TCJs that do not meet the criterion.

(2) aSFs nucleate a distance $x_{0}$ away, along the midline axis, from the center of a nucleating TCJ. $x_{0}$ is equal to the cortex thickness (Fig. S8S).

(3) aSF endpoints move at constant velocity $v_{x}$, along the midline axis, towards the cell center. The speed of aSFs along the midline is independent of cell area (Fig. S8Q).

(4) If a cell has only one breaking TCJ (Fig. S8M), we assume the aSF breaks as it approaches its endpoint approaches that TCJ, a distance $\Delta x$ before reaching the breaking TCJ as it links up to cortex in the neighboring cell. (We expect $\Delta x$ to be approximately equal to, but not necessarily exactly equal to, the cortex thickness.) The aSF lifetime is determined by the separation between the breaking TCJ and the nucleating TCJ.

(5) Suppose the cell has more than one breaking TCJ (i.e., at least one breaking TCJ on both sides of the cell so that each aSF tip can reach a breaking TCJ). It is possible that the aSF could break when one aSF tip reaches its breaking TCJ. It is also possible that the aSF could break as the other aSF tip reaches its breaking TCJ. We assume that the aSF lifetime is determined by the average of the separations between the nucleating TCJ and each of the two breaking TCJs.

As suggested by this model, we characterize aSF movement as one-dimensional (i.e., along the a-p axis) since the aSFs are aligned with the m-l axis (Fig. 1D). Given the geometry of the cell at the time of aSF breakage and the TCJ from which the aSF nucleated, our model makes a specific prediction about the lifetime of each aSF. The lifetime of each aSF is determined by how far it moves from its nucleating TCJ before breakage (Fig. S8R). Given this phenomenological picture of aSF dynamics (Fig. S8T), we proceed to estimate model parameters from time-lapse imaging.

\section{E. Experimental estimates of parameters of aSF motion and breakage}

Before we calculate how much of the scaling of aSF number with cell apical area can be explained by this model, we specify estimates for the model parameters. We have experimental estimates for the criteria for nucleating TCJs and breaking TCJs (Fig. S7F,G and Fig. S8M). This means that given a snapshot of a cell, we can predict at which TCJs aSFs nucleate and at which TCJs aSFs break. We additionally have an estimate of $x_{0}$, which is the cortex thickness (Fig. S8S).

There are three parameters in the model, including $k_{n u c}$ and $v_{x}$ and $\Delta x$, that we have not yet estimated from time-lapse imaging. $k_{n u c}$ is the nucleation rate per nucleating TCJ. $v_{x}$ is the speed of aSF tips along the a-p axis. The average signed distance between the leading aSF tip and its breaking TCJ at the

time of breakage is $\Delta x$ (positive if the aSF breaks as its endpoint approaches the breaking TCJ, negative if the aSF breaks after its endpoint passes the TCJ) (see Fig. S8T). As we will see in Section 2F, $k_{n u c}$ and $v_{x}$ 
appear in the model as a single parameter, $\frac{k_{n u c}}{v_{x}}$. We estimate $k_{n u c}$ by counting aSF nucleation events across 51 cells over 2 hours. For each of these cells, we calculate the average number of nucleating TCJs (i.e., TCJs with bisector orientations that satisfy the aSF nucleation criterion) over the same time period. The average aSF nucleation rate per nucleating TCJ is $0.031 \pm 0.001 \mathrm{~min}^{-1}$ (mean $\pm \mathrm{SEM}$ ). The average aSF speed along the a-p axis for tracked aSFs for these 51 cells is $0.111 \pm 0.003 \frac{\mu m}{\min }$ (mean $\pm \mathrm{SEM}$ ) (Fig. S8Q). Thus, $\frac{k_{n u c}}{v_{x}}$ is $0.29 \pm 0.01 \mu m^{-1}$ (mean \pm SEM).

For the remaining parameter $\Delta x$, we do not have an estimate from time-lapse imaging. We will leave $\Delta x$ as a free parameter in Section $2 \mathrm{~F}$. After using this parameter to fit our model to wild-type aSF counts per cell, we will check whether the best-fit value of the $\Delta x$ is physically reasonable. Equipped with this model (from Section 2D) for which all but one parameter is constrained, we will compare our model's prediction for the scaling of aSF number with cell apical area to the experimentally observed scaling of aSF number with cell apical area.

\section{F. Explanatory power of cell-autonomous model: scaling of aSF number with apical area}

Based on our knowledge that aSFs tend to nucleate at TCJs and break at TCJs, we seek to quantify what fraction of the scaling of aSF number with cell apical area can be explained by our phenomenological model. From skeletonized images of the region of interest at $26 \mathrm{~h} \mathrm{APF,} \mathrm{we} \mathrm{define} \mathrm{the} \mathrm{TCJs} \mathrm{and} \mathrm{cell-cell}$ junctions. To these still packings, we apply the model which we summarize in Section 2E. Let us denote the separation (along the a-p axis) between nucleating TCJ (indexed by $j$ ) and the first breaking TCJ by $x_{n u c-\text { break } 1}{ }^{j}$. Let us denote separation (along the a-p axis) between the nucleating TCJ and the second breaking TCJ by $x_{n u c-b r e a k 2}{ }^{j}$.

Suppose a cell has only one breaking TCJ. Our model predicts that the lifetime of an aSF nucleated by TCJ (indexed by $j$ ) is equal to $\frac{\max \left(x_{n u c-b r e a k 1}{ }^{j}-\left(\Delta x+x_{0}\right), 0\right)}{v_{x}}$. The mean aSF number in cell $i$ from nucleating $\operatorname{TCJ} j$ is:

$\overline{N_{l \jmath}}=\frac{k_{n u c} * \max \left(x_{n u c-b r e a k 1}{ }^{j}-\left(\Delta x+x_{0}\right), 0\right)}{v_{x}}$

Suppose the cell has more than one breaking TCJ (i.e., at least one breaking TCJ on both sides of the cell so that each aSF tip can reach a breaking TCJ). Our model predicts that the lifetime of an aSF nucleated by TCJ (indexed by $j$ ) is equal to $\frac{\max \left(\operatorname{mean}\left(x_{n u c-b r e a k 1}{ }^{j}, x_{n u c-b r e a k 2}{ }^{j}\right)-\left(\Delta x+x_{0}\right), 0\right)}{v_{x}}$. The mean aSF number in cell $i$ from nucleating TCJ $j$ is:

$\overline{N_{l j}}=\frac{k_{n u c} * \max \left(\operatorname{mean}\left(x_{n u c-\text { break }}{ }^{j}, x_{n u c-b r e a k 2}{ }^{j}\right)-\left(\Delta x+x_{0}\right), 0\right)}{v_{x}}$

The mean aSF number in cell $i$ (from all its nucleating TCJs) is:

$\overline{N_{\iota}}=\sum_{\text {nucleating TCJ }(j)} \overline{N_{\iota j}}$

Changing $\left(\Delta x+x_{0}\right)$ in the model shifts the mean aSF number per cell up or down with little effect on the slope of aSF number versus cell apical area. We are mostly interested in the slope of aSF number versus cell apical area rather than in uniform shifts of aSF number per cell; we leave $\left(\Delta x+x_{0}\right)$ as a free parameter. For 25,000 cells in 35 distinct samples, we calculate the sum of squared errors as a function of $\left(\Delta x+x_{0}\right)$. The value of $\left(\Delta x+x_{0}\right)$ that minimizes the sum of squared errors (cell-by-cell) between the 
model prediction and actual aSF counts is $1.01 \pm 0.01 \mu \mathrm{m}$ (error estimated via bootstrapping; bootstrp; MATLAB 2016B Statistics and Machine Learning Toolbox, MathWorks). This is approximately two times the cortex thickness (i.e., $\left.\left(\Delta x+x_{0}\right) \approx 2 x_{0}\right)$; the experimental value of the cortex thickness is approximately $0.51 \pm 0.12 \mu \mathrm{m}$ (Fig. S8S).

Using the value of $\left(\Delta x+x_{0}\right)$ that minimizes the sum of squared errors, we plot the mean aSF number predicted by our model for all cells of a given apical area in Fig. 5D. The mean aSF number for a given apical area is an average over the sum in Eq. 21 for all cells of a given apical area. Using the same technique for determining incremental contributions of variables to explaining variance as above (58), we find that this model, with the nucleating and breaking TCJ cutoffs constrained by experimental data, explains approximately 75 percent of the scaling of aSF number with cell apical area at $26 \mathrm{~h}$ APF (Fig. 5D). This cell-autonomous model predicts that the slope of aSF number versus cell apical area should be

approximately $0.05 \frac{a S F}{\mu m^{2}}$, whereas the observed scaling is approximately $0.06 \frac{a S F}{\mu m^{2}}$ (Fig. 5D).

The fundamental physical content of this model is that TCJs set the average aSF number per cell on a largely cell-autonomous basis. The role that a given TCJ plays in determining the number of aSFs in a cell depends on the orientation of its bisector relative to the uniaxial stress. If the bisecting vector of a TCJ points along the a-p axis (Fig. S7A,B), the TCJ is a nucleation site for aSFs in the cell. If the bisecting vector of a TCJ points along the $\mathrm{m}-1$ axis (Fig. S7A,B), the TCJ is a site at which aSFs break in the cell. The assumptions that aSF number is set entirely cell-autonomously likely underlies the model's inability to completely explain the variation in the data. Indeed, the model is blind to many details of the mechanical environment of cells, which we expect to affect MyoII accumulation and activity (87-90). Nevertheless, our model, based solely on individual cell geometries, with one free parameter explains approximately 75 percent of the experimentally observed scaling of aSF number with cell apical area (Fig. 5D). Encouragingly, the value of the fit parameter which best fits the experimental data is approximately equal to the cortex thickness (Fig. S8S), suggesting a physical picture in which aSFs break near TCJs as they link up with cortex in a neighboring cell (Fig. S8T).

\section{Experimental tests of cell-autonomous, geometric model}

\section{A. Testing the model by varying TCJ number: Comparing clone boundary to clone bulk}

Since the geometric model helps to explain the scaling of aSF number with cell apical area in wildtype dorsal thoraces at $26 \mathrm{~h}$ APF (Fig. 5D), we seek to test the model directly. The geometric model, based on the role of TCJs in aSF nucleation and breakage, suggests that manipulating the number of TCJs could affect aSF number, independent of cell apical area. To change the average number of TCJs in a set of cells, we generate clones of cells that are larger than the wild-type cells (Fig. 5E). Large cells in a tissue of small cells have more TCJs, on average, than large cells in a tissue of large cells $(27,91)$.

To accomplish an increase in cell apical area, we prevent division in a subset of cells $\left(t r b l^{U P}\right)$. To study the effect of a differing number of TCJs per cell at approximately the same cell apical area (Fig. S9A), we compare $t r b l^{U P}$ cells at clone boundaries to $t r b l^{U P}$ cells in the clone bulk (Fig. 5E).

Having generated and segmented images of tissues harboring large $t r b l^{U P}$ clones, we divide the clone cells into two groups, $t r b l^{U P}$ cells which contact at least one wild-type cell (466 cells, $\mathrm{trbl}^{U P}$ clone border cells) and $t r b l^{U P}$ cells which contact no wild-type cells (683 cells, $t r b l^{U P}$ clone bulk cells). Both groups have approximately the same apical area per cell; the mean difference in cell apical area is $5.0 \pm$ $1.5 \mu \mathrm{m}^{2}$ (mean \pm SEM) (Fig. S9A). Although $t r b l^{U P}$ border cells are of approximately the same apical size as $t r b l^{U P}$ bulk cells, $t r b l^{U P}$ border cells have roughly one more TCJ per cell than $t r b l^{U P}$ bulk cells (Fig. S9D,E). 
Since our $\operatorname{trbl}^{U P}$ manipulations lead to more TCJs per cell in border cells than in bulk cells, we ask whether we observe differences in aSF number per cell between border and bulk cells as a result of this difference in cell geometry. As shown in Fig. 5G, without controlling for differences in cell apical area, the mean difference in aSF number per cell between $t r b l^{U P}$ border cells and $t r b l^{U P}$ bulk cells is $0.4 \pm 0.1$ aSF (mean $\pm \mathrm{SEM}$ ). The $t r b l^{U P}$ group with more TCJs has more aSFs per cell (Fig. S9B-E).

We would like to know if the observed mean aSF number difference between clone boundary and clone bulk in the $t r b l^{U P}$ cells can explained solely by the small difference in mean cell apical area between $t r b l^{U P}$ border cells and $t r b l^{U P}$ bulk cells. If not, we would like to know if our geometrical model explains the difference in mean aSF number per cell between the two groups of $\mathrm{trbl}^{U P}$ cells (Fig. 5G).

To test whether the observed mean aSF number difference between $t r b l^{U P}$ clone boundary cells and $\mathrm{trbl}^{U P}$ clone bulk cells can be explained by a difference in mean apical area, we perform an analysis of covariance (58). We linearly regress the aSF count in each cell on two independent variables. One independent variable is categorical, zero for $t r b l^{U P}$ cells in the clone bulk and one for $t r b l^{U P}$ cells at the clone boundaries. The other independent variable is the cell apical area. The 95\% confidence interval for the scaling of aSF number with cell apical area is $\left(0.028 \frac{a S F}{\mu m^{2}}, 0.035 \frac{a S F}{\mu m^{2}}\right)$ (Fig. S9B,C).

The $95 \%$ confidence interval for the regression coefficient of the categorical variable is $(0.10 \mathrm{aSF}$, $0.43 \mathrm{aSF}$ ). Thus, controlling for cell apical area differences, $t r b l^{U P}$ cells at the clone boundaries have, on average, about 0.25 more aSF than $t r b l^{U P}$ cells in the clone bulk (Fig. S9B,C). The p-value for the $t$-statistic that the regression coefficient of the categorical variable is non-zero is 0.001 (fitlm; MATLAB 2016B Statistics and Machine Learning Toolbox, MathWorks) (58).

We now turn to the cell-autonomous, geometric model to see if the model predicts the difference in mean aSF number between $t r b l^{U P}$ border cells and $t r b l^{U P}$ bulk cells. We simulated aSF nucleation and breakage in the geometric model on cell sizes and shapes taken from our images of $t r b l^{U P}$ clones. Performing an analysis of covariance on the model predictions, we linearly regress the predicted aSF number count in each cell on the same two independent variables. The $95 \%$ confidence interval for the regression coefficient of the categorical variable is $(0.05 a S F, 0.21 \mathrm{aSF})$ (Fig. S9F,G); controlling for cell apical area differences, the model predicts that $t r b l^{U P}$ boundary cells have on average 0.15 aSF more than $t r b l^{U P}$ bulk cells.

Our geometric model thus accounts for approximately $60 \%$ of the observed effect (i.e., $60 \%$ of the increased aSF number per cell in $t r b l^{U P}$ boundary cells relative to the $t r b l^{U P}$ bulk cells). For the model prediction, the $\mathrm{p}$-value for the $t$-statistic that the regression coefficient for the categorical variable is nonzero is 0.001 (Fig. S9F,G) (fitlm; MATLAB 2016B Statistics and Machine Learning Toolbox, MathWorks) (58). In summary, increasing the number of TCJs per cell in $t r b l^{U P}$ border cells, while controlling for differences in cell apical area, increases the mean aSF number in a statistically significant way, in agreement with expectations from our geometrical model.

In conclusion, our model, applied to the still images of $t r b l^{U P}$ clone cells, predicts the following $t r b l^{U P}$ effect: increasing the number of TCJs in a cell tends to increase the number of aSFs in a cell. This is due to an increase in predicted aSF nucleation rate (Fig. 5F) without any difference in predicted aSF lifetime between $t r b l^{U P}$ border cells and $t r b l^{U P}$ bulk cells (Fig. 5H). Although our model is cell-autonomous and relies on a few simple assumptions, motivated by the time-lapse imaging of aSF nucleation and breakage, the phenomenological model is powerful enough to predict the effect of changing the number of TCJs at fixed cell apical area in these $t r b l^{U P}$ clone experiments. 


\section{B. Ortho-elongated cells tend to have more aSFs, as predicted by cell-autonomous, geometric model}

The number of aSFs per cell is determined by a balance of aSF nucleation and aSF breakage. In Section $3 \mathrm{~A}$, we tested our model in $t r b l^{U P}$ cells by analyzing whether increasing the number of TCJs would lead to an increase in nucleation rate and thus of aSF number, as predicted by our model (Fig. 5F,I). We now test our model on cell geometries that are expected to promote long aSF lifetimes. In our geometrical model, aSF lifetime is proportional to the distance between a nucleating TCJ and breaking TCJs along the a-p axis (Fig. S8R,T). To test this second characteristic of our model, in this section we seek a group of cells that are elongated orthogonal to the uniaxial tensile stress such that expected nucleating TCJs and breaking TCJs are far from each other (Fig. 5J).

We define ortho-elongated cells based on the moment of inertia tensor. First, we compute the geometric center of the cell, $\left(x_{\text {geocent }}, y_{\text {geocent }}\right)$. Then, for each pixel $i$ within the cell, we compute $\Delta x_{i}=$ $x_{i}-x_{\text {geocent }}, \Delta y_{i}=y_{i}-y_{\text {geocent }}$. Since, by convention, the m-l axis corresponds to the y-axis, orthoelongated cells are those with $\frac{\sum_{\text {ipixelswithincell }\left(\Delta y_{i}\right)^{2}}}{\sum_{\text {ipixelswithincell }\left(\Delta x_{i}\right)^{2}}} \leq 1$; elongated cells are those with $\frac{\sum_{\text {i pixels within cell }}\left(\Delta y_{i}\right)^{2}}{\sum_{\text {i pixels within cell }\left(\Delta x_{i}\right)^{2}}}>1$.

In comparing ortho-elongated cells to elongated cells, we control for cell apical area and for the number of nucleating TCJs per cell (proportional to the predicted aSF nucleation rate per cell). We pair each ortho-elongated cell (indexed by $i$ with number of nucleating TCJs $n_{i}$ and cell apical area $A_{i}$ ) with the elongated cell (indexed by $j$ ) with the same number of nucleating TCJs and the closest possible area (i.e., we find the elongated cell $j$ with the smallest $\left|A_{j}-A_{i}\right|$ among all those with $n_{j}=n_{i}$ ). Once elongated cell $j$ has been matched with ortho-elongated cell $i$, we remove elongated cell $j$ from the pool so that it cannot be paired with any other ortho-elongated cells. Since this procedure depends on the order in which we loop through the list of ortho-elongated cells, we repeat the procedure with random permutations of the looping order several times, generating different paired sets. The results in Fig. $5 \mathrm{~K}-\mathrm{O}$ are from a representative randomly generated paired set. We compare the aSF number per ortho-elongated cell to the aSF number per elongated cell. We find that the group of ortho-elongated cells has more aSFs per cell than the group of elongated cells (Fig. 5K).

Since we control for the number of nucleating TCJs (Fig. 5L), the predicted aSF nucleation rates per cell are the same for the group of elongated cells and the group of ortho-elongated cells. We verified using the experimentally determined elongated and ortho-elongated cell shapes that, as expected, our model does indeed predict a longer aSF lifetime and, thus, more aSFs in the ortho-elongated cells. Lastly, to check whether ortho-elongated cells have more aSFs simply as a result of having more total MyoII (not explicitly included in our phenomenological model of nucleation and breakage), we measured the integrated MyoII pool at apical regions in a group of 151 randomly selected ortho-elongated cells and their paired elongated cells (chosen as just described). Whereas the numbers of aSFs per cell are different in the two cell populations, the apical pool of MyoII is similar between the two populations (Fig. S9H-J). Therefore, cells of a given size but different cell geometry can have a distinct number of aSFs even if they have a similar MyoII level.

The analyses of ortho-elongated cells and $t r b l^{U P}$ cells (Section 3A) demonstrate that at fixed cell apical area, the number and spatial distribution of TCJs significantly impact the number of aSFs per cell. 


\section{Possible biophysical mechanisms for the distinction between nucleating and breaking TCJs}

In this section, we state hypotheses that could account for the fact that aSF nucleation rate depends so strongly on TCJ bisector orientation (see Section 2A). We explain in Section 4A how a model for aSF nucleation based solely on the orientation of the two adjacent edges that meet at the TCJ disagrees with our empirical observations. Then, in Section 4B, we use our observations of cell geometries under increasing uniaxial tensile stress to motivate a proposal in which aSFs appear in response to increases in the opening angle of a TCJ over time. Additionally, we discuss specific proteins that could plausibly be responsible for aSF nucleation at TCJs. Although we do not attempt to verify these proposed mechanisms directly in the current paper, they provide at least some suggestion of how our observed dependence of nucleation and breakage on TCJ orientation could plausibly arise.

\section{A. aSF nucleation model based solely on edge orientation}

The role of TCJs in aSF nucleation arises quite naturally in a very simple model of aSF formation inspired by the observation that aSF tend to peel off from the cortex and by similar models of membrane adhesion to surfaces (92). We imagine that the actin, Myosin II, and other proteins that will form the aSF can exist in two states, as a proto-aSF still bound to the rest of the junctional cortex or as a detached, fullyformed aSF. When the proto-aSF is attached to the rest of the cortex, the proto-aSF gains an adhesion energy $-\sigma$ per unit length which favors its remaining attached. At the same time, both the proto-aSF and the detached aSF are contractile and thus can be modeled as having a line tension $\lambda$ that favors peeling from the cortex in order to shorten the aSF: Peeling allows the (proto-)aSF to become shorter because, instead of following the curved cell-cell junction, upon peeling it can form a straight line between its two attachment points. Thus, we expect peeling to be most favorable where junctions are most highly curved and the decrease in line tension energy from peeling is correspondingly largest. As TCJs are regions of high junctional curvature, it, thus, makes sense that aSFs tend to nucleate at TCJs. Interestingly, this explanation of aSF nucleation at TCJs due to high junctional curvature is supported by the fact that aSFs also nucleate at cell-cell junctions which become curved during division of a neighbouring cell prior to completion of cytokinesis, as described in Section 2A.

Specifically, suppose an aSF nucleates from the two edges adjacent to a TCJ and moves in the direction of the TCJ bisecting vector. The change in energy due to peeling of a segment of proto-aSF initially of length $2 s$ is:

$\Delta U=U_{f}-U_{i}=2 s \sin \left(\frac{\alpha}{2}\right) \lambda-2 s(\lambda-\sigma)$.

$\Delta U<0 \Leftrightarrow \sin \left(\frac{\alpha}{2}\right)<\frac{\lambda-\sigma}{\lambda}=1-\frac{\sigma}{\lambda}$.

For $\Delta U<0$, it is energetically favorable for aSFs to nucleate from the cortex because the energetic favorability of length minimization overcomes the energetic cost of breaking attachments with the rest of the cell cortex. In this simplest model, nucleation thus occurs when the TCJ opening angle $\alpha$ is small enough.

A natural extension of this simple model, to account for externally imposed anisotropic tensile stress, is to make $\lambda$ and/or $\sigma$ dependent on the orientations of the two adjacent edges relative to the a-p axis (which, recall, is perpendicular to the uniaxial stress). For the sake of simplicity, let us consider cases in which the bisector of the pair of adjacent edges points either along the a-p axis or along the m-l axis. We will compare TCJs in the bin indicated by the red dot to the bin indicated by the blue dot in Fig. S10A-B. Those in the bin indicated by the red dot have opening angle larger than $\frac{\pi}{2}$ (i.e., $\alpha_{r e d}=\frac{\pi}{2}+\epsilon ; \epsilon>0$ ). Those in the bin indicated by the blue dot have opening angle smaller than $\frac{\pi}{2}$ (i.e., $\left.\alpha_{\text {blue }}=\frac{\pi}{2}-\epsilon ; \epsilon>0\right)$. We observe that 
TCJs in the bin indicated by the red dot do nucleate aSFs, while TCJs in the bin indicated by the blue dot do not nucleate aSFs.

The notable fact about the TCJs in these two bins is that (assuming the same value of $\epsilon$ in both cases) the two edges adjoining each red TCJ are at the same angles with respect to the a-p axis as the two edges adjoining each blue TCJ, as shown in Fig. S10C,C'. Thus, even if the parameters $\lambda$ and $\alpha$ depend on edge orientation, they will be the same for the TCJs in the red and blue bins. Let us call the orientation of each of the edges with respect to the a-p axis $\delta=\delta_{\text {red }}=\delta_{\text {blue }}$. Although the edges have the same orientation with respect to the a-p axis, the TCJs in the red bin have a larger opening angle than the TCJs in the blue bin. This means that $\sin \left(\frac{\alpha_{\text {red }}}{2}\right)>\sin \left(\frac{\alpha_{\text {blue }}}{2}\right)$. Based on Eq. 23, it is thus impossible for the TCJ defined by the red edges to nucleate aSFs (i.e., to have $\left.\sin \left(\frac{\alpha_{\text {red }}}{2}\right)<1-\frac{\sigma(\delta)}{\lambda(\delta)}\right)$ if the TCJ defined by the blue edges does not (i.e., $\left.\sin \left(\frac{\alpha_{\text {blue }}}{2}\right)>1-\frac{\sigma(\delta)}{\lambda(\delta)}\right)$, because $\sin \left(\frac{\alpha_{\text {red }}}{2}\right)>\sin \left(\frac{\alpha_{\text {blue }}}{2}\right)$. Regardless of the choice of orientational dependence for $\lambda$ and/or $\sigma$, this edge-orientation-dependent framework hence cannot explain the fact that TCJs in the red bin nucleate aSFs while the TCJs in the blue bin do not nucleate aSFs (Fig. S10A-C')

\section{B. Dependence of opening angle on bisector orientation in tissue under uniaxial stress}

It, thus, remains an open question how TCJs with certain bisector orientations "know" that they are supposed to nucleate aSFs while TCJs with the opposite orientations "know" that they are not. Here, we argue that one appealing possibility is that the cortex (or related factors) in the vicinity of the TCJ could have some memory of its history of deformation, so that aSF nucleation depends on the rate of change of TCJ opening angle rather than on instantaneous edge orientations. We also discuss specific molecular, mechanosensitive mechanisms that could be responsible for nucleation at TCJs for which the bisectors point perpendicular to the uniaxial stress.

To quantify the response of the tissue to the uniaxial tensile stress, we measure the correlation between TCJ bisecting angle $\theta$ and opening angle $\alpha$ (Fig. S10D). In wild-type tissues at $18 \mathrm{~h} \mathrm{APF}$, before the increase in tensile stress, $\alpha$ does not depend on $\theta$. Indeed, the mean opening angle, regardless of $\theta$, is

approximately $\frac{2 \pi}{3}$, as one would expect in a mechanically isotropic tissue. Then, as the imposed uniaxial tensile stress increases from $18 \mathrm{~h} \mathrm{APF}$ to $26 \mathrm{~h} \mathrm{APF} \mathrm{(Fig.} \mathrm{1B,} \mathrm{S1B,C),} \mathrm{the} \mathrm{opening} \mathrm{angle} \alpha$ between adjacent edges develops a dependence on TCJ bisecting angle $\theta$ (Fig. S10D).

If a TCJ's bisector into a cell points mostly along the a-p axis (Fig. S10C), the opening angle $\alpha$ tends to increase in response to increasing tensile stress (Fig. S10D). If the bisector points mostly along the $\mathrm{m}-1$ axis (Fig. S10C'), $\alpha$ instead tends to decrease in response to increasing tensile stress (Fig. S10D). Our observations of aSF nucleation in time-lapse images reveal that TCJ bisector orientation is strongly predictive of aSF nucleation (Fig. S7B-F); for TCJs with bisectors pointing along the a-p axis, we hypothesize that aSFs nucleate at certain TCJs because their opening angle has recently increased in response to increased tensile stress.

Unlike a mechanism that depends only on instantaneous edge orientation, such a history-dependent mechanism is, thus, at least qualitatively consistent with the observation that a TCJ's nucleation rate depends primarily on its bisector orientation $\theta$. Since the opening angle between adjacent edges increases in response to uniaxial tensile stress, we hypothesize that aSF nucleation could occur downstream of recruitment of medial MyoII at TCJs. 
Recruitment of MyoII to TCJs in Drosophila epithelia is well-documented (48, 76, 77). For example, during the stretching of the Drosophila pupal wing epithelium due to forces from the hinge, MyoII forms ring-like structures around TCJs during cell rearrangement (77). Similarly, in germ-band extension during cell neighbor exchanges (i.e., T1 transitions), pulses of medial MyoII near TCJs generate the lengthening of new cell-cell junctions (76). In some existing mechanical models of simple epithelia, Myosin II recruitment to cell-cell junctions occurs when the junctions experience strain (88). One could extend such models of active tension networks to allow recruitment of MyoII specifically at TCJs; this recruitment to TCJs would occur as the opening angle between adjacent junctions increases, straining actomyosin filaments at the TCJ.

Furthermore, we foresee that the dynamics of TCJ proteins might be modulated in response to uniaxial tissue stress. For example, the conserved Sidekick (Sdk) protein is localized at TCJs in multiple Drosophila epithelial tissues; an increase in junction tension or a change in cell geometry was proposed to modulate its level at TCJs (93-95). This suggests that the composition of TCJs might depend on the orientation and anisotropy of tissue stress. Therefore, the detailed analysis of TCJ protein dynamics is a promising avenue to shed light on how the TCJ bisector angle defines nucleating and breaking TCJs. 


\section{Fig. S1. Tissue mechanical stress and aSF organization.}

Unless otherwise stated, protein distributions and quantifications are reported for the region boxed in Fig. $1 \mathrm{~A}$ at $26 \mathrm{hAPF}$.

(A-A") Picture of the Drosophila melanogaster pupa (A); pupal dorsal thorax labeled by ECadherin:3xGFP (E-Cad:3xGFP) imaged at 26 hAPF by multi-position confocal microscopy (A'); Inset in (A') shows the cell resolution capacity of multi-position confocal microscopy and close-up picture of the adult dorsal thorax (notum, A"). Orange region in A delineates the pupal dorsal thorax (notum) shown in A'. Black dashed box: posterior and central region where aSFs form along the $m-1$ axis and corresponding region in the adult (A'). Green dashed box: lateral regions where aSFs formed along the a-p axis. White lines: midline.

(B) Medial-lateral (m-l) tissue elongation (blue) and inferred junctional mechanical stress anisotropy (red; positive values indicate tensile stress is higher along the m-l axis) in the region boxed in A'. N: number of animals at each timepoint.

(C) Graph of the tissue recoil velocity along the a-p axis (red) and m-l axis (blue) between $14 \mathrm{hAPF}$ and 28 hAPF. Blue asterisks: calculated for the comparison of the recoiled velocities measured at $18 \mathrm{hAPF}$ and at a later given timepoint for a-p ablation, $p$-values $<0.05$ from 22 hAFP. Red asterisks: calculated for the comparison of the recoiled velocity measured along the a-p and m-1 tissue axes, $p$-values $<10^{-5}$ from 22 hAFP. N: minimum number of ablations at each timepoint and orientation.

(D) Schematic of aSF (green) ablation and recoil velocity measurement. Upon aSF laser ablation, the $l_{0}$ and $l_{t}$ distances (blue lines: corresponding to the distances at $\mathrm{t}=0$ and $\mathrm{t}=1.3 \mathrm{~s}$ ) were used to calculate the aSF recoil velocity.

(E,E') E-Cad:3xmKate2, nls:mRFP and MyoII:3xGFP distributions at the level of the AJ (E) and along the cell a-b axis (E'). Yellow arrowheads in E indicate the position of a-b section shown in E'. Conversely, the yellow arrowheads in $\mathrm{E}^{\prime}$ indicate the $\mathrm{a}-\mathrm{b}$ position of the section shown in $\mathrm{E}$.

(F-F'') Dlg:YFP and MyoII:3xmKate2 distributions at the level of the AJ (F), of the septate junction (F') and along the a-b axis ( $\left.F^{\prime \prime}\right)$. Yellow arrowheads in $\mathrm{F}$ indicate the position of a-b section shown in $\mathrm{F}$ '. Yellow and blue arrowheads in F' indicate the a-b position of the section shown in F and F', respectively. (G) Graph of the intensities of Actn:GFP (blue) and PH:GFP (grey) along AJ centred at the aSF tip (arrowhead). n: number of junctions. $p$-values $<10^{-5}$. Statistical comparisons were performed between the intensities at the aSF tip ( $\pm 0.54 \mu \mathrm{m}$ around the aSF tip) and the AJ intensities away from the aSF tip. The percentage to the right represents the Actn:GFP (blue) and PH:GFP (grey) signal increase near the aSF tip relative to AJ regions away from aSF tip (see M\&M).

(H-Q) Localization of LifeAct:GFP and MyoII:3xmKate2 (H), Zyx:ChFP and MyoII:3xGFP (I), Ena:GFP and MyoII:3xmKate2 (J), Vinc:GFP and MyoII:3xmKate2 (K), Kst:YFP and MyoII:3xmKate2 (L), Zasp52:GFP and MyoII:3xmKate2 (M), Pax:YFP and MyoII:3xmKate2 (N), Cher:YFP and MyoII:3xmKate2 (O), Rhea:GFP and MyoII:3xmKate2 (P) and Mys:GFP and MyoII:3xmKate2 (Q).

(R) E-Cad:3xmKate2 and MyoII:3xGFP distributions within the green region boxed in $\mathrm{A}^{\prime}$ at $26 \mathrm{hAPF}$ (right). Arrowheads: aSFs.

(S) Rose plot of the orientation of aSFs (green) and inferred main stress axis (mean \pm STD in red and light red, respectively) in the lateral green dashed regions in A' at 26 hAPF. n: aSF number. This illustrates that in another region of the tissue aSFs are oriented along the main axis of tissue stress.

Scale bars: $200 \mu \mathrm{m}$ (A, A' '), $50 \mu \mathrm{m}$ (A'), $5 \mu \mathrm{m}$ (R), $2 \mu \mathrm{m}$ (E, E', F, F'” and H). 
Statistical tests: Kruskal-Wallis test with Conover post hoc (C) and Kruskal-Wallis test (G). ns: not significant. Statistically significant differences $(p$-value $<0.05)$ are indicated by using one asterisk. In the case of multiple pairwise comparisons, only the maximum $p$-values (of the set of asterisked comparisons) are reported. 


\section{Fig. S2. aSFs and mechanical stress.}

To test whether an increase in mechanical stress would be sufficient to promote aSF formation, Myosin II phosphatase regulatory subunit loss of function clones $\left(\mathrm{mbs}^{R N A i}\right)$ were generated to locally increase cell contractility. The number of aSFs per cell in the two layers of wt cells surrounding the mbs ${ }^{R N A i}$ clones were then compared to the number of aSFs in the two layers of wt cells surrounding $w^{R N A i}$ control clones at 20 $h A P F(D, E, F)$.

Protein distributions and quantifications are reported for the region boxed in Fig. 1A.

(A-A') MyoII:3xGFP and E-Cad:3xmKate2 in control mirr-G4>LifeAct:GFP (A) and in mirr$G 4>$ zip $^{D N}: Y F P$ tissues at $26 \mathrm{hAPF}\left(\mathrm{A}^{\prime}\right)$. The dotted green lines and lateral green bars delineate roughly the lateral domain where mirr-G4 is expressed (A, A'). The medial domain where mirr-G4 is not expressed is less elongated along the $\mathrm{m}-1$ axis in mirr-G4>zip ${ }^{D N}: Y F P$ in agreement with the reduced mechanical stress measured in the central domain (Fig. 1I). mirr-G4 specifically drives expression in the lateral domain of the tissue (96)

(B-C') Schematic of the pupal m-l compression. Arrowheads indicate the compression orientation (B and C'). Distributions of MyoII:3xGFP in uncompressed tissue (C) and in a tissue compressed by around 20\% along the $\mathrm{m}-1$ axis $\left(\mathrm{C}^{\prime}\right)$ at $26 \mathrm{hAPF}$. The cyan lateral margins illustrate the magnitude of $\mathrm{m}-1$ compression (C').

(D, E) MyoII:3xmKate2 and Jub:GFP in $w^{R N A i}$ control cell clones (D, blue) or in $m b s^{R N A i}$ cell clones (E, blue) and surrounding wt cells (D, E) at $20 \mathrm{hAPF}$. Dashed blue lines delineate the boundary between the $w^{R N A i}$ and wt cells (D) or the boundary between $m b s^{R N A i}$ and wt cells (E). aSFs in the 2 layers of wt cells (surrounding the clone) are indicated by arrowheads.

(F) Graph of aSF number per cell in the two layers of wt cells surrounding $w^{R N A i}$ or $m b s^{R N A i}$ clones at 20 hAPF. n: number of aSFs per cell; $p$-value $<10^{-2}$.

Scale bars: $20 \mu \mathrm{m}(\mathrm{A}, \mathrm{C})$ and $5 \mu \mathrm{m}(\mathrm{D}, \mathrm{E})$.

Statistical tests: Kruskal-Wallis test $(\mathrm{F})$. Statistically significant differences ( $p$-value $<0.05)$ are indicated by using one asterisk. 


\section{Fig. S3. aSF scaling and its role in cell and tissue elongation.}

Unless otherwise stated, protein distributions and quantifications are reported for the region boxed in Fig. $1 \mathrm{~A}$ at $26 \mathrm{hAPF}$.

(A) Distribution of MyoII:3xGFP in $a^{a t n^{I G I}}$ cells (orange dashed outline) marked by the lack of expression of Ubi-nls:mRFP (not shown) and in surrounding control cells. Arrowheads: aSFs.

(B) Graph of the normalized aSF number per cell in control and actn cells. n: number of cells; $p$-value $<10^{-5}$.

(C) Graph of the normalized cell elongation of control and actn cells. n: number of cells; $p$-value $<10^{-5}$.

(D,D') Graph of the MyoII:3xmKate2 cortical levels in $w^{R N A i}$ control and $a c t n^{R N A i}$ clones at $18 \mathrm{hAPF}$ and 26 hAPF. n: number of cells.

(E,E') Graph of aSF nucleation rate (E) and lifetime (E', $p$-value $\left.<10^{-5}\right)$ in $w^{R N A i}$ control and $a c t n^{R N A i}$ cells. The reduced number of aSFs in actn cells is therefore mainly caused by a reduced lifetime of the aSFs. n: number of cells.

(F) Graph of aSF number per cell in $p n r-G 4>w^{d s R N A}$ (control) versus $p n r-G 4>a c t n^{d s R N A}$ tissues. n: number of cells; $p$-value $<10^{-5}$.

(G) Graph of the cell elongation in $p n r-G 4>w^{d s R N A}$ (control) versus $p n r-G 4>a c t n^{d s R N A}$ tissues. n: number of cells; $p$-value $<10^{-5}$.

(H-H') $p n r-G 4>w^{d s R N A}(\mathrm{H})$ and $p n r-G 4>a c t n^{d s R N A}\left(\mathrm{H}^{\prime}\right)$ adult scutella. $\mathrm{h}$ is the length of the scutellum along the a-p axis (blue line). $\mathrm{v}$ is the maximum length of the scutellum along the $\mathrm{m}-\mathrm{l}$ axis (green line). (H') Graph of the scutellum elongation (v/h) in control $p n r-G 4>w^{d s R N A}$ and $p n r-G 4>a c t n^{d s R N A}$ animals. n: number of animals; $p$-value $<10^{-5}$.

(I) Graph of the m-l tissue recoil velocities upon a-p laser ablation in $p n r-G 4>w^{d s R N A}$ (control) versus $p n r-$ $G 4>a c t n^{d s R N A}$. pnr-G4 specifically drives expression in the medial domain of the tissue (97). n: number of ablations.

(J) Schematic of AJ (purple) ablation and recoil velocity measurement. Upon AJ laser ablation, the $l_{0}$ and $l_{t}$ distances (orange lines, corresponding to the distances at $\mathrm{t}=0$ and $\mathrm{t}=1.3 \mathrm{~s}$ ) were used to calculate the AJ recoil velocity.

(K) Radar plot of the recoil velocity upon ablation of either a junction (blue) or an aSF (pink) as a function of the orientation of the ablated structure relative to the a-p axis at 26 hAPF. n: number of ablations.

(L) Graph of the recoil velocity $(\mu \mathrm{m} / \mathrm{s})$ upon aSF ablation as a function of cell apical area. n: number of cells.

$(\mathbf{M}, \mathbf{N})$ Graph of the number of aSFs per cell as a function of cell apical area (M) or volume (N). n: number of cells.

(O) Graphs of the cell apical area (left) and aSF number per cell (right) $1 \mathrm{~h}$ before and $1 \mathrm{~h}$ after telophase in control cells ( $p$-values $<10^{-5}$ for area and $p$-values $<10^{-5}$ for aSF number) and aurora $B^{R N A i}\left(\right.$ aur $B^{R N A i}$ ) cells, ns for area and number of aSFs). n: number of cells.

(P) Graph of cell elongation as a function of cell apical area in $w^{R N A i}$ and $a c t n^{R N A i}$ clones at 26 hAPF. n: number of cells; $p$-value $<10^{-5}$.

(Q) Graph of difference in cell elongation upon ablation of all aSFs in a given cell as a function of its apical area. Differences were measured around $9.5 \mathrm{~s}$ after ablation. $\mathrm{n}$ : number of cells. $p$-value $<0.05\left(25-35 \mu \mathrm{m}^{2}\right.$ and $+35 \mu \mathrm{m}^{2}$ ) for differences with the first bin.

Scale bars: $5 \mu \mathrm{m}(\mathrm{A})$ and $100 \mu \mathrm{m}(\mathrm{H})$

Statistical tests: Kruskal-Wallis tests (B-G and H'-I), Kruskal-Wallis tests with Conover post hoc (O and Q) and Ancova tests for difference in regression slopes (P). r: Pearson correlation coefficient. ns: not 
significant. Statistically significant differences $(p$-value $<0.05)$ are indicated by one asterisk. 
Fig. S4. Theoretical modelling of cell elongation as a function of tissue stress anisotropy and aSF number.

To gain a physical understanding of the contributions of aSFs to epithelial cells under anisotropic mechanical stress, we consider regular hexagonal epithelia under uniaxial tensile stress (by convention, along the vertical axis). The regular hexagonal epithelia are organized into one of two orientations, called the cable-forming (CFO) and non-cable-forming (NCFO) orientations, relative to the uniaxial stress $(A-C)$. Tensile forces on cell-cell junctions and on aSFs allow these epithelial cells to resist elongation (D-H). Given that no cell has an infinite pool of MyoII, there is a finite bound on the stress anisotropy that the cells can resist $\left(\sigma_{\max }>\sigma_{D} \equiv \sigma_{y y}-\sigma_{x x}>0\right)$. We ask whether cells resist elongation more or less effectively by redistributing junctional MyoII to aSFs $(D-H)$. The vertex model with aSFs predicts that for cells to remain regular hexagons under uniaxial stress, the number of aSFs, or the total aSF tension, per cell must scale linearly with the square root of the cell apical area (I). Larger cells must compensate for the greater distance between edges (orthogonal to the uniaxial stress) by increasing the aSF number, or total aSF tension, per cell.

(A) Schematic of hexagonal epithelium in the cable-forming orientation (CFO) with aSFs (green). The epithelial geometry is characterized by two edge lengths $\left(l_{1}\right.$ and $\left.l_{2}\right)$ and one angle $(\theta)$. Edges of length $l_{1}$ have tension $\gamma_{1}$; edges of length $l_{2}$ have tension $\gamma_{2}$. Total aSF tension per cell is $\gamma_{f}$.

(B) Schematic of hexagonal epithelium in the non-cable-forming orientation (NCFO) with aSFs (green). The epithelial geometry is characterized by two edge lengths $\left(l_{3}\right.$ and $\left.l_{4}\right)$ and one angle $(\phi)$. Edges of length $l_{3}$ have tension $\gamma_{3}$; edges of length $l_{4}$ have tension $\gamma_{4}$. Total aSF tension per cell is $\gamma_{f}$.

(C) Schematic of stress anisotropy calculation in hexagonal epithelium from (B). Using linear cuts of the epithelium along the axes parallel (magenta dashed line) or perpendicular (blue dashed line) to the aSFs, we sum projections (perpendicular to the cut) of tensile forces from edges and aSFs to calculate a tensile force per length (i.e., 2D stress) along the cut.

(D-E) Schematics of cell shapes and edge tensions for fixed stress anisotropy $\left(\frac{\sigma_{D}}{\sigma_{\max }}\right.$ indicated in each panel) in the CFO (top) and NCFO (bottom). Edge and aSF widths correspond to the magnitude of tension. Cells with more aSFs (vertical lines in each cell) have a larger total aSF tension $\gamma_{f}$. As the number of aSFs increases, cell elongation in the direction of the imposed tension decreases. The blue dashed lines are regular hexagons (see SI equations 11, 12).

(F) Graph of cell elongation normalized by the elongation $S_{\text {reg,hex }}$ of a regular hexagon $\left(\frac{S}{S_{\text {reg,hex }}}\right)$ as a function of stress anisotropy $\left(\frac{\sigma_{D}}{\sigma_{\max }}\right)$. We compare cells in the CFO without aSFs to cells in the NCFO with total aSF tension $\left(\gamma_{f}\right)$.

(G-H) Plot of normalized cell elongation $\left(\frac{S}{S_{\text {reg,hex }}}\right)$ as a function of the total aSF tension $\left(\gamma_{f}\right)$ and stress anisotropy $\left(\frac{\sigma_{D}}{\sigma_{\max }}\right)$ for cells in the CFO and NCFO. Tension is scaled such that $\gamma_{f}=1$ corresponds to the edge tension in the case of isotropic stress without aSFs. Note that large total aSF tensions (upper left-hand quadrant of plot) generate cells which are elongated orthogonal to the uniaxial stress. The elongation measure $S$ does not distinguish between elongation along the uniaxial stress and elongation orthogonal to the uniaxial stress.

(I) Plot of aSF number per cell (at fixed tension per aSF) as a function of the square root of cell apical area in the vertex model. For this plot, $\sigma_{D}=\frac{2}{3} \sigma_{\max }$ and the tension per aSF is equal to one third of the edge 
tension in the case of isotropic stress without aSFs.

(J) Graph of the cell apical area as a function of the square root of the cell apical area between 10 and $40 \mu \mathrm{m}$, illustrating the linear relationship between these quantities for the sample of wt cells used in our analysis of aSFs in time-lapse imaging. $n$ : cell number.

r: Pearson correlation coefficients. 


\section{Fig. S5. Modulation of Hippo/Yki signalling by mechanical stress.}

In order to quantify the intensity of the Jub and Wts distributed in clusters at different developmental timepoints or in different experimental conditions, we implemented a method based on the Fiji FindFoci plug-in (52) allowing cluster identification (E-E','). To determine whether Jub clustering could modulate Hippo signaling, we developed an optogenetic approach to induce Jub clustering independently of aSF formation. The Cry2Olig:ChFP protein forms clusters upon blue-light illumination (22). We generated a Cry2Olig:ChFP:Jub as well as a control Cry2Olig:ChFP, and expressed them ubiquitously. Blue-light illumination was sufficient to induce the clustering of both Cry2Olig:ChFP and Cry2Olig:ChFP:Jub in the absence of aSFs. While the light-induced clustering of control Cry2Olig:ChFP promotes neither Wts:GFP clustering nor change of Yki transcriptional activity, the light-induced clustering of Cry2Olig:ChFP:Jub leads to the formation of Wts:GFP clusters colocalizing with Cry2Olig:ChFP:Jub and to an increase in Yki transcriptional activity as measured using the dIAPl-nls:GFP (55) (H-J and Movie S8).

Unless otherwise stated, protein distributions and quantifications are reported for the region boxed in Fig. $1 \mathrm{~A}$ at $26 \mathrm{hAPF}$.

(A) Graph of the intensities of Wts:GFP (purple), Jub:GFP (orange), Actn:GFP (blue) and PH:GFP (grey) along AJ centred at the aSF tip (arrowhead). n: number of junctions. $p$-values $<10^{-5}$ for Wts:GFP ,Jub.GFP and Actn:GFP. Statistical comparisons were performed between the intensities at the aSF tip $( \pm 0.54 \mu \mathrm{m}$ around the aSF tip) and the AJ intensities away from the aSF tip. Percentages represent the signal increase near the aSF tip relative to AJ regions away from aSF tip for the different tagged proteins (see $\mathrm{M} \& \mathrm{M}$ ).

(B-C') Jub:GFP (B,B'), Wts:GFP (C,C') and MyoII:3xmKate2 (B-C') distributions at the level of the AJ $(\mathrm{B}, \mathrm{C})$ and along the cell $\mathrm{a}-\mathrm{b}$ axis $\left(\mathrm{B}^{\prime}, \mathrm{C}^{\prime}\right)$. Yellow arrowheads in $\mathrm{B}$ and $\mathrm{C}$ indicate the position of a-b section shown in B' and C', respectively. Conversely, the yellow arrowheads in B' and C' indicate the a-b position of the section shown in $\mathrm{B}$ and $\mathrm{C}$, respectively.

(D) Graph of the a-b position of the Jub:GFP, Wts:GFP, and $\alpha$ Catenin:GFP ( $\alpha$ Cat:GFP) clusters relative to position of maximal E-Cad:3xGFP signal illustrating that Jub:GFP and Wts:GFP clusters are localized at the level of the AJ.

(E-E'") Schematic of protein cluster identification and ratio ${ }^{\text {in/out }}$ of cluster measurement. Fluorescence signals were sum projected from $8 \mathrm{z}$-sections above to $8 \mathrm{z}$-sections below the AJs, which corresponds to 3.2 $\mu \mathrm{m}$ in total (E,E'). The sum projected image was used to determine: (i) The mean fluorescence intensity in cluster (IC) : Clusters were automatically identified using the Fiji FindFoci plug-in (52) (E'). The resulting mask was used to measure the mean intensity in each cluster $(I c)$ and the area occupied by each cluster $(A c)$; (ii) The mean intensity along the AJ, (Iaj): the AJ cell contour was selected using Fiji segmented line tool (blue, E',') and used to measure (Iaj) and the total area of the cell junction ( $A a j)$; (iii) The mean fluorescent background intensity ( $I b)$ was measured by selecting the apical medial region of the cells (red, E'"). The

signal in cluster was defined as in ${ }^{\text {cluster }}=\frac{\sum_{\text {each cluster }((I c-I b) * A c)}}{(I a j-I b) * A a j}$ and signal on the junction as out cluster $^{\text {s }}=1-$ in $^{\text {cluster }}$. The ratio ${ }^{\text {in/out }}$ of cluster is defined as $\frac{\text { in }_{\text {cluster }}}{\text { out cluster }}$.

(F) Graph of the number of Jub:GFP and Wts:GFP clusters per cell at 18 hAPF (low stress) and 26hAPF (high stress). n: number of cells; $p$-values $<10^{-4}$ for the difference between 18 hAPF and 26 hAPF for both Jub:GFP and Wts:GFP.

(G) Graph of colocalization (Pearson Correlation Coefficient) between Jub:mKate2 and Jub:GFP or between Jub:mKate2 and Wts:GFP at 18 hAPF (low stress) and 26 hAPF (high stress). Pearson coefficients were normalized by the mean of the Pearson coefficient between Jub:GFP and Jub:mKate2 at 18 hAPF. n: number of cells; Jub:mKate2 and Jub:GFP colocalization: ns; Jub:mKate2 and Wts:GFP colocalization: $p$ - 
value $<10^{-5}$.

(H,H') Distributions of Cry2Olig:ChFP and Wts:GFP at the time of blue light illumination at $\mathrm{t}=0 \mathrm{~s}(\mathrm{H})$ and at $\mathrm{t}=950 \mathrm{~s}$ upon blue light illumination every $50 \mathrm{~s}\left(\mathrm{H}^{\prime}\right)$. See also Movie S8A.

(I,I') Distributions of Cry2Olig:ChFP:Jub and Wts:GFP at the time of blue light illumination at $\mathrm{t}=0 \mathrm{~s}$ (I) and at $\mathrm{t}=950 \mathrm{~s}$ upon blue light illumination every $50 \mathrm{~s}$ (I'). See also Movie S8B.

(J) Graph of the intensity of the dIAP1-nls:GFP Yorkie reporter in tissues expressing Cry2Olig:ChFP or Cry2Olig:ChFP:Jub in dark (ns) or light $(p$-value $<0.01)$ conditions. Fluorescence intensities were measured at $0 \mathrm{~h}$ (beginning of the experiment) and $4 \mathrm{~h}$. The fluorescence intensities measured at $4 \mathrm{~h}$ were divided by the fluorescence intensities measured at $0 \mathrm{~h}$, and the resulting ratios were normalized by the mean of the ratios calculated for Cry2Olig: $\mathrm{ChFP}$ of each condition. Clustering was induced by two initial steps of high blue laser illumination (491 nm, interval $5 \mathrm{~min}$ ), and then was maintained at low laser power for GFP imaging, every $5 \mathrm{~min}$. N: number of experiments.

(K,K') Distributions of Wts:CitFP (green, K and fire, K') and MyoII:3xGFP during aSF displacement. Yellow arrowheads: aSF (K); light green arrowheads: Wts:CitFP cluster (K').

(L) Wts:CitFP (L) before and after ( $\mathrm{t}=210 \mathrm{~s})$ ablation of the aSF labelled by MyoII:3xmKate2. Time was set to 0 just before ablation (ablation at $\mathrm{t} \sim 0.01 \mathrm{~s}$ ). Arrowheads: cluster prior to and after ablation.

(M) Graph of Wts:CitFP cluster intensities in mock ablated aSF (control) and upon aSF ablation. n: number of ablations, $p$-value $<10^{-2}$ after timepoint $120 \mathrm{~s}$.

(N,O) Distributions of Jub:GFP (N) and Wts:CitFP (O) in $a c t n^{R N A i}$ cells (marked by the expression of Caax:tBFP, not shown) outlined by orange dotted line and surrounding control cells.

$(\mathbf{P}, \mathbf{Q})$ Graph of the cortical Jub:GFP (P) and Wts:GFP (Q) normalized integrated intensity in $w^{R N A i}$ control and $a c t n^{R N A i}$ cells. Integrated intensities of the $w^{R N A i}$ control or $a c t n^{R N A i}$ cells were normalized by the mean integrated intensity of surrounding cells not juxtaposed with the $w^{R N A i}$ control and $a c t n^{R N A i}$ cells, respectively. (R) Graphs of the number of Jub:GFP and Wts:CitFP clusters in $w^{R N A i}$ control and $a c t n^{R N A i}$ clones at 26 hAPF. n: number of cells; $p$-values $<10^{-3}$ for Jub:GFP and Wts:CiFP.

(S) Graph of normalized colocalization (Pearson Correlation Coefficient) of Jub:mKate2 and Wts:GFP in $w^{R N A i}$ control and in $a c t n^{R N A i}$ clones at 26h APF. n: number of cells; $p$-value $<10^{-4}$.

(T) Graph of ban-nls:GFP intensity in control and $a c t n^{I G l}$ cells at $26 \mathrm{hAPF}$. Average intensities were normalized by the mean intensities of control cells. $\mathrm{N}$ : number of animals; $p$-value $<10^{-2}$.

(U) dIAP1-nls:GFP Yorkie reporter normalized intensity in $w^{R N A i}$ control and $a c t n^{R N A i}$ clones at $26 \mathrm{hAPF}$. $\mathrm{N}$ : number of animals; $p$-value $<10^{-3}$.

(V) Graph of cell division rate between 16 and $40 \mathrm{hAPF}$ in $p n r-G 4>w^{d s R N A}$ (control) and pnr-G4>actn ${ }^{d s R N A}$ tissues. At the central and posterior region of the tissue (box in Fig. 1A), cell proliferation occurs as two successive waves $(4,10)$. The first wave occurs between 18 and $24 \mathrm{hAPF}$, while the second one occurs after $24 \mathrm{hAPF}$. This second wave of division corresponds to cells that have progressed through the cell cycle under higher mechanical stress. The reduction of Actn function leads to a specific decrease in the number of cell divisions during this second wave. Average number of cells used per animal in $w^{R N A i}$ is 218 and in $\operatorname{actn}^{R N A i}$ is 148 . N: number of animals. $p$-value $<10^{-2}$.

Scale bars: $2 \mu \mathrm{m}\left(\mathrm{B}-\mathrm{C}^{\prime}, \mathrm{H}, \mathrm{K}\right.$ and L), $5 \mu \mathrm{m}(\mathrm{N})$.

Statistical tests: Kruskal-Wallis tests (A, F, G, J and P-U), Kruskal-Wallis tests with Conover post hoc (D, $\mathrm{M})$ and mixed-ANOVA test (V). ns: not significant. Statistically significant differences $(p$-value $<0.05)$ are indicated by one asterisk. 


\section{Fig. S6. Hippo/Yki scaling with cell apical area.}

Unless otherwise stated, protein distributions and quantifications are reported for the region boxed in Fig. $1 \mathrm{~A}$ at $26 \mathrm{hAPF}$.

(A) Graph of the intensity of $d I A P 1-n l s: G F P$ and Ubi-nls:GFP as a function of apical cell area. This further confirms the scaling between cell apical area and Yorkie transcriptional activity. n: number of cells. $p$ value $<10^{-5}$.

(B) Graph of Wts:GFP ratio $^{\text {in/out }}$ of cluster as a function of apical cell size at $18 \mathrm{hAPF}$ and $26 \mathrm{hAPF}$. $\mathrm{n}$ : number of cells; $p$-value $<10^{-4}$.

(C) Graph of Wts:CitFP and Jub.GFP ratio ${ }^{\text {in/out }}$ of cluster as a function of apical cell size at 18 hAPF. n: number of cells; $p$-value $<0.05$.

(D) Graph of the number of Jub:GFP clusters as a function of apical cell size in $w^{R N A i}$ control and $a c t n^{R N A i}$ clones at 26 hAPF. $\mathrm{n}$ : number of cells; $p$-value $<10^{-5}$.

(E) Graph of apical cell area versus ban-nls:GFP intensity in $w^{R N A i}, \operatorname{actn}^{R N A i}$, aurB $B^{R N A i}$ and $s v n^{R N A i}$ clones. Number of cells (n) used to calculate cell apical area (and clustering of Jub:GFP, in Fig S6F) and number of animals (N) for ban-nls:GFP; $w^{R N A i}$ (n: 140 and N: 8), $\operatorname{actn}^{R N A i}$ (n: 125 and N: 13), aurB ${ }^{R N A i}$ (n: 45 and N: 9) and $s u v^{R N A i}$ (n: 140 and N: 10). Dotted line is the regression line for the $w^{R N A i}$, aurB $B^{R N A i}$ and $s v n^{R N A i}$ data points.

(F) Graph of Jub:GFP ratio ${ }^{\text {in/out }}$ of cluster versus ban-nls:GFP intensity in $w^{R N A i}, a^{2} t^{R N A i}$, aurB $B^{R N A i}$ and $s v n^{R N A i}$ clones. Number of cells (n) used for Jub:GFP clustering (and cell apical area, in Fig. S6E) and number of animals (N) for ban-nls:GFP; $w^{R N A i}$ (n: 140 and N: 8), $\operatorname{actn}^{R N A i}$ (n: 125 and N: 13), aurB ${ }^{R N A i}$ (n: 45 and N: 9) and $s v n^{R N A i}$ (n: 140 and N: 10). Dotted line is the regression line for $w^{R N A i}, a c t n^{R N A i}$, aurB $B^{R N A i}$ and $s v n^{R N A i}$ data points.

Statistical tests: Ancova tests for difference in regression slopes (A-D). ns: not significant. Statistically significant differences $(p$-value $<0.05)$ are indicated by one asterisk. 


\section{Fig. S7. Quantitative characterization of aSF nucleation at TCJs.}

More than 95 percent of aSFs nucleate at a TCJ (n=624, Fig. 5C). For a given cell's shape, we would like to predict which TCJs are sites of aSF nucleation for that cell. When an aSF nucleates at a TCJ, we calculate both the TCJ's opening angle and the orientation of its bisector in that cell relative to the a- $p$ axis $(A)$; these are the angles for the pair of adjacent edges that meet at the TCJ $(A)$. We compare the geometric properties of nucleating TCJs to the geometric properties of the set of all TCJS (C-D). The TCJ bisector orientation is strongly predictive of aSF nucleation; nucleating TCJs are TCJS with a bisector pointing mostly along the a-p axis (E-G). We check that the nucleation rate per cell is proportional to the number of nucleating TCJS (H). After verifying that the number of nucleating TCJs increases with cell apical area (I), we explore whether large cells have more aSFs because they have more nucleating TCJs. Using an aSF-nucleating $T C J$ criterion derived from the data, we find that a "nucleation-only" model (in which the nucleation rate per cell is proportional to the number of nucleating TCJS) generates a weak scaling of aSF number with cell apical area $(J)$.

Unless otherwise stated, protein distributions and quantifications are reported for the region boxed in Fig. $1 \mathrm{~A}$ at $26 \mathrm{hAPF}$.

(A) Illustration of an aSF nucleating at a nucleating TCJ (red arrowhead) and peeling from the cortex until the aSF tips reach breaking TCJs (blue arrowhead) and of the TCJ opening angle $\alpha$ and TCJ bisector orientation $\theta$ with respect to the a-p axis. Orange arrows indicate the TCJ bisector, which makes an angle $\theta$ with the a-p axis $\left(\theta \approx 5^{\circ}\right.$ for the nucleating TCJ at right; $\theta \approx 80^{\circ}$ for the breaking TCJ at bottom). Cyan arcs indicate opening angle.

(B) Cumulative distribution of TCJ bisector orientations for TCJs where aSFs are observed to nucleate (red) and break (blue). See Section 2C of SI for our criterion for aSF breakage at a TCJ. n: number of nucleation and breakage events.

(C) Grey-coded plot of fraction of TCJs with opening angle $(\alpha)$ and bisector orientation $(\theta)$. n: pairs of adjacent edges ( 3 pairs of adjacent edges per individual TCJ).

(D) Grey-coded plot of fraction of aSF nucleation events as a function of opening ( $\alpha$ ) and bisector ( $\theta$ ) TCJ angles. n: number of nucleation events.

(E) Grey-coded plot of nucleation rate as a function of opening $(\alpha)$ and bisector $(\theta)$ TCJ angles. This relative nucleation rate is the ratio of the data in (D) to the data in (C). The white region with dashed lines corresponds to TCJs which are rarely observed (less than $0.01 \%$ in (C)). n: number of nucleation events.

(F) Empirical cumulative distribution of the aSF nucleation data as a function of bisector orientation $\theta$ (red curve). The aSF-nucleating TCJ cutoff (dashed magenta line) is based on the ninety-fifth percentile of aSF nucleation events. $n$ : number of nucleation events.

(G) Graph of the nucleation rate as a function of TCJ bisector orientation $\theta$. Within each bin of $\theta$, we compute the ratio of the number of nucleation events (related to Fig. S7D) to the fraction of adjacent edge pairs (related to Fig. S7C) in that bin and normalize all bins by the largest ratio. The vertical (dashed magenta) line denotes the aSF nucleation cutoff in (E). n: number of nucleation events.

(H) Graph of aSF nucleation rate $\left(\mathrm{h}^{-1}\right)$ as a function of the average number of nucleating TCJs over $2 \mathrm{~h}(25-$ 27 hAPF). n: number of cells.

(I) Graph of number of TCJs per cell (grey) and number of aSF-nucleating TCJ per cell (red) as a function of cell apical area. n: number of cells.

(J) Graph of experimental (grey) and predicted (red) aSF number per cell as a function of cell apical area. 
The red line is the result of the "nucleation-only" model. n: number of cells.

r: Pearson correlation coefficient. ns: not significant. 


\section{Fig. S8. Quantitative characterization of aSF breakage at TCJs.}

To determine where aSFs break, we track aSF positions from midlife to breakage in E-Cad:3xmKate2 and MyoII:3xGFP time-lapse images. Since no aSF is perfectly aligned with the uniaxial stress, for each aSF we can define a leading tip and a lagging tip. The leading tip is the aSF tip that has moved farther from the nucleating TCJ along the a-p axis, and the lagging tip is the other aSF endpoint $(A, B)$. To quantify the aSF position, we measure the distance along the a-p axis between the nucleating TCJ and the leading aSF tip (B). We, first, show that aSFs break near the cell centroid and that aSFs are closer to the cell centroid at breakage than at midlife (C,D). Furthermore, aSFs are more likely to break near TCJs than would be expected solely from a bias towards breaking near the cell centroid (E-G, J, K). We assign an aSF breakage event to a specific TCJ, if the distance between the leading aSF tip and the TCJ is less than the cortex thickness, estimated in (S). We determine the geometric characteristics of these aSF-breaking TCJS (L,M). After defining an aSF-breaking TCJ criterion (M), we develop a phenomenological model in which aSFs move at a constant speed (Q) from aSF-nucleating TCJS to aSF-breaking TCJs. As validated by experimental data $(R)$, our model assumes that aSF lifetime is proportional to how far each aSF moves from nucleation to breakage (T).

Unless otherwise stated, protein distributions and quantifications are reported for the region boxed in Fig. $1 \mathrm{~A}$ at $26 \mathrm{hAPF}$.

(A) Distribution of E-Cad:3xmKate2 and MyoII:3xGFP immediately before aSF breakage. In this example, both aSF tips are attached to TCJs at the time of aSF breakage.

(B) Schematic for calculation of aSF penetration distance (orange dashed line) and cell centroid position (red dashed line) along the a-p axis relative to the nucleating TCJ (red arrow). The aSF is green. aSF tips are indicated by yellow arrows. The cell centroid is indicated by a blue dot.

(C) Graph of aSF penetration distance as a function of the separation between the aSF's nucleating TCJ and the cell centroid position at aSF midlife. The dashed line is the line of best fit, which indicates that the extent of aSF penetration at midlife depends on the distance between the nucleating TCJ and the cell centroid ( $p$ value $<10^{-5}$ ). n: number of aSFs.

(D) Graph of aSF penetration distance as a function of the separation between the aSF's nucleating TCJ and the cell centroid position at aSF breakage. The dashed line is the line of best fit, indicating that the extent of aSF penetration at the time of breakage depends on the distance between the nucleating TCJ and the cell centroid $\left(p\right.$-value $\left.<10^{-5}\right)$. aSFs penetrate farther by the time of breakage than at midlife (see SI, Section $2 \mathrm{C}$ ). n: number of aSFs.

(E,F) Two examples of aSFs at the time of breakage in two distinct cells. In both cases, the aSF breaks near the cell centroid and is aligned with two TCJs. The red dashed line is the a-p distance between the aSFnucleating TCJ (red arrows) and the cell centroid (blue circles), which is plotted in (D, x-coordinate). The orange dashed line is the distance between the position of the aSF nucleation and the leading aSF tip, which is plotted in (D, y-coordinate). Although the cell in $(\mathrm{E})$ and $(\mathrm{F})$ are distinct, the distances between the nucleating TCJ and the cell centroid are the same for both examples.

(G) To illustrate our procedure for defining a distance $\delta^{a S F-T C J}$ swapped for each breakage event (see SI), we swap the aSF from the cell in (E) into the cell in (F). $\delta^{a S F-T C J}$ swapped (yellow line) is the distance along the a-p axis from the breaking TCJ to the leading tip of the swapped aSF.

(H) Histogram of distance between the leading tip of each aSF and its nearest TCJ at the time of aSF midlife. n: number of aSFs.

(I) Histogram of distance between the leading tip of each swapped aSF and its nearest TCJ in the cell into 
which it has been swapped at aSF midlife. n: number of aSFs.

(J) Histogram of distance between the leading tip of each aSF and its nearest TCJ at the time of aSF breakage. n: number of aSFs.

(K) Histogram of distance between the leading tip of each swapped aSF and its nearest TCJ in the cell into which it has been swapped at the time of aSF breakage. n: number of aSFs.

(L) Grey-coded plot of the fraction of aSF breakage events as a function of the opening $(\alpha)$ and bisector angle $(\theta)$ of the breaking TCJ. n: number of aSFs.

(M) Empirical cumulative distribution of aSF breakage events as a function of TCJ bisector angle $\theta$ (blue curve). Dashed magenta line: cutoff above which we define TCJs to be aSF-breaking TCJs. n: number of aSFs.

(N-P) Graphs of average aSF number per cell $(\mathrm{N})$, aSF nucleation rate $(\mathrm{O})$ and aSF lifetime $(\mathrm{P})$ measured between 25 and 27 hAPF as a function of cell apical area. Dashed lines: best fit linear regressions; n: number of cells.

(Q) Graph of average aSF velocity per cell $\left(\mu \mathrm{m} \cdot \mathrm{min}^{-1}\right)$ as a function of cell apical area. $\mathrm{n}$ : number of cells.

(R) Graph of average aSF lifetime ( $\mathrm{min}$ ) as a function of average aSF total distance travelled $(\mu \mathrm{m})$ from the nucleating TCJ. n: number of cells.

(S) Graph of E-Cad:3xmKate2 and MyoII:3xGFP intensities as a function of the distance to a nucleating TCJ. E-Cad:3xmKate2 and MyoII:3xGFP intensity profiles were measured along a line of 5 pixels wide $(0.13 \mu \mathrm{m}$ per pixel), which passes through the nucleating TCJ ( 2 min before aSF nucleation) orthogonal to the nascent aSF. Positive distances correspond to pixels within the cell in which an aSF will nucleate; negative distances correspond to pixels on the other side of the TCJ. We selected pixels that are between $0.5 \mu \mathrm{m}$ and $2.0 \mu \mathrm{m}$ from the nucleating TCJ. We normalized both E-Cad:3xmKate2 and MyoII:3xGFP intensity profiles. Maximum intensity peaks were found for E-Cad:3xmKate2 (magenta arrow) and MyoII:3xGFP on the average intensity profiles. Junctional cortex thickness was estimated as the double of the distante given via a cross-correlation between average intensity profiles of E-Cad:3xmKate2 and MyoII:3xGFP (junctional cortex thickness estimation $0.52 \mu \mathrm{m}$ ). Alternatively, junctional cortex thickness was estimated by measuring MyoII:3xGFP and E-Cad:3xmKate2 intensity profiles for each nucleating TCJ. In this case, junctional cortex thickness is estimated as the average distance between the peak of E-Cad:3xmKate2 signal (vertical dotted magenta line) and the position corresponding to $66 \%$ of the maximal MyoII:3xGFP signal (vertical dotted blue line). Both measurements result in similar junctional cortex thickness values: $0.52 \mu \mathrm{m}$ versus $0.51 \pm 0.12 \mu \mathrm{m}$. n: number of TCJs.

(T) Schematic for model of aSF lifetimes as a function of cell geometry. First, the aSF (green) nucleates a short distance (burgundy; shown in inset) from its nucleating TCJ (red arrow). The aSF moves away from its nucleating TCJ until its tips (green arrows) reach breaking TCJs (blue arrows). The aSF breaks when its tips (green arrows) are within a short distance (orange; shown in inset) from breaking TCJs. (Note that $x_{0}<$ 0 would correspond to aSF breakage after passing breaking TCJs.) The predicted aSF lifetime is proportional to the distance (along the a-p axis) that the aSF moves, on average, from its nucleation site to its breakage site (bracketed red dotted line).

Statistical tests: $F$-test (C,D). r: Pearson correlation coefficient. 
Fig. S9. Analyses of $\operatorname{trbl}^{U P}$ cell clones and MyoII pool in elongated and ortho-elongated cells.

Unless otherwise stated, protein distributions and quantifications are reported for the region boxed in Fig. $1 \mathrm{~A}$ at $26 \mathrm{hAPF}$.

(A) Graph of cell apical area for $t r b l^{U P}$ border and bulk cells. n: number of cells; $p$-value $<10^{-3}$.

(B) Graph of the experimental number of aSFs as a function of cell apical area in $t r b l^{U P}$ border and bulk cells. n: number of cells.

(C) Graph of the experimental number of aSFs and cell apical area in $t r b l^{U P}$ border and bulk cells. n: number of cells; $p$-value $<10^{-2}$. For each group, the dashed line represents the scaling of aSF number with cell area given by the analysis of covariance. The scaling of aSF number with cell area is $0.031 \frac{a S F}{\mu m^{2}}$.

(D) Graph of number of TCJs per cell for $t r b l^{U P}$ border and bulk cells. n: number of cells; $p$-value $<10^{-5}$.

(E) Graph of the number of TCJs per cell as function of cell apical area in $t r b l^{U P}$ border and bulk cells. n: number of cells.

(F) Graph of the predicted number of aSFs as a function of cell area in $t r b l^{U P}$ border and bulk cells. n: number of cells.

(G) Graph of the predicted number of aSFs and cell apical area in $\mathrm{trbl}^{U P}$ border and bulk cells. n: number of cells; $p$-value $<10^{-2}$. For each cell group, the dashed line represents the scaling of aSF number with cell area given by the analysis of covariance. The scaling of aSF number with cell area is $0.030 \frac{\mathrm{aSF}}{\mu \mathrm{m}^{2}}$.

(H-J) Graphs of the number of aSFs per cell $\left(\mathrm{H} ; p\right.$-value $\left.<10^{-2}\right)$, apical cell area (I) and of the MyoII apical pool $(\mathrm{J})$ in elongated and ortho-elongated cells. n: number of cells.

Statistical tests: Kruskal-Wallis tests (A,D,H-J) and Ancova tests for difference in regression intercepts (C and G). ns: not significant. Statistically significant differences ( $p$-value $<0.05$ ) are indicated by one asterisk. 


\section{Fig. S10. Dependence of aSF nucleation on TCJ bisector orientation.}

To investigate why TCJ bisector orientation is predictive of aSF nucleation at TCJS $(A, B)$, we consider a model in which an aSF nucleates at a TCJ when the decrease in line tension energy overcomes the increase in energy due to detachment from the junctional cortex. To account for mechanical anisotropy, we allow both the line tension energy and the attachment energy to depend on the orientations of cell-cell junctions relative to the a-p axis. We find that this model is incapable of explaining why aSFs nucleate at TCJs like (C) while aSFs do not nucleate at TCJs like (C'). Searching for a possible physical reason for the dependence of aSF nucleation on TCJ bisector orientation, we analyse the relationship between TCJ opening angle and TCJ bisector orientation under uniaxial stress (D). Under increases in tensile uniaxial stress, TCJs for which bisectors point perpendicular to the a-p axis ( $C^{\prime}$ ) tend to close (i.e., decrease in opening angle), and TCJs for which bisectors point along the a-p axis (C) tend to open (i.e., increase in opening angle). We propose a hypothesis in which aSFs nucleate at TCJs due to increases in TCJ opening angle; aSF nucleation could occur via a mechanosensitive mechanism as actomyosin filaments near a TCJ experience strain during increases in the TCJ opening angle. We also foresee that the TCJ composition might be modulated by mechanical stress since the level of the TCJ protein Sidekick was recently reported to be modulated by junction tension or cell geometry (93-95).

(A) Grey-coded plot of fraction of TCJs with opening angle $(\alpha)$ and bisector orientation $(\theta)$ (adapted from Fig. S7C). Red dot indicates a group of TCJs for which the bisector points along the a-p axis. Blue dot indicates a group of TCJs for which the bisector points perpendicular to the a-p axis. n: pairs of adjacent edges ( 3 pairs of adjacent edges per TCJ).

(B) Grey-coded plot of nucleation rate as a function of opening $(\alpha)$ and bisector $(\theta)$ TCJ angles (adapted from Fig. S7E). The red dot indicates a group of TCJs for which the bisector points along the a-p axis and at which aSFs nucleate. The blue dot indicates a group of TCJs for which the bisector points perpendicular to the a-p axis and at which no aSFs nucleate. n: number of nucleation events.

(C) Schematic of TCJ for which the bisector points along the a-p axis and with an opening angle $\alpha$ larger than $\frac{\pi}{2}$ (i.e., $\varepsilon>0$ ). The angle of each cell-cell junction relative to the a-p axis is $\delta$.

(C') Schematic of TCJ for which the bisector points perpendicular to the a-p axis and with an opening angle $\alpha$ smaller than $\frac{\pi}{2}$ (i.e., $\varepsilon>0$ ). The angle of each cell-cell junction relative to the a-p axis is $\delta$.

(D) Graph of opening angle $\alpha$ as a function of bisector orientation $\theta$ at $18 \mathrm{~h} \mathrm{APF,} 22 \mathrm{~h} \mathrm{APF}$, and $26 \mathrm{~h} \mathrm{APF}$. $\mathrm{n}$ : pairs of adjacent edges ( 3 pairs of adjacent edges per TCJ). 
Table S1: aSF component localizations and functions.

\begin{tabular}{|c|c|c|c|}
\hline $\begin{array}{l}\text { Proteins and } \\
\text { References }^{(a)}\end{array}$ & $\begin{array}{l}\text { Drosophila } \\
\text { names }\end{array}$ & aSF localizations & $\begin{array}{c}\text { loss of function } \\
\text { phenotypes }\end{array}$ \\
\hline PDLIM1/LDB3 $(98,99)$ & Zasp52 & Fibers and Tips & No loss of aSF \\
\hline Zyxin (100) & Zyxin & Fibers and Tips & No loss of aSF \\
\hline$\alpha-\operatorname{Actinin}(101-103)$ & Actn & Fibers and Tips & Loss of aSF \\
\hline$\beta$-Spectrin (104) & Kst & Fibers and Tips & $\begin{array}{c}\text { Junction and aSF } \\
\text { breakage }\end{array}$ \\
\hline Filamin A (105) & Cher & Fibers and Tips & No loss of aSF \\
\hline$\beta$-Integrin (106) & Mys & Tips & No loss of aSF \\
\hline Paxilin (100) & Pax & Tips & No loss of aSF \\
\hline Talin (107) & Rhea & Tips & No loss of aSF \\
\hline $\operatorname{Vasp}(108,109)$ & Ena & Tips & No loss of aSF \\
\hline Vinculin (110) & Vinc & Tips & No loss of aSF \\
\hline Anillin (111) & Scra & - & Not tested \\
\hline $\operatorname{Arp3}(112)$ & Arp3 & - & Not tested \\
\hline Dystrophin (113) & Dys & - & Not tested \\
\hline Fascin $(81)$ & Fascin & - & Not tested \\
\hline Fimbrin $(102,103,114)$ & Fim & - & Not tested \\
\hline mDiaphanous 2 (115) & Dia & - & Not tested \\
\hline p130CAS (116) & p130CAS & - & Not tested \\
\hline PDLIM1/LDB3 $(98,99)$ & Zasp66 & - & Not tested \\
\hline Septin 2 (117) & Sep2 & - & Not tested \\
\hline Septin $4(118)$ & Sep4 & - & Not tested \\
\hline $\begin{array}{l}\text { Tropomyosin } 1 \text { (119, } \\
\text { 120) }\end{array}$ & Tm1 & - & Not tested \\
\hline Tropomyosin 2 (120) & $\operatorname{Tm} 2$ & - & Not tested \\
\hline
\end{tabular}

(a): References describing the localization and the function of the associated proteins.

(b): To avoid identifying indirect regulators of aSF formation, only components harboring aSF localization were qualitatively tested. At least two distinct dsRNA lines either from Drosophila TRiP (121) or VDRC (122) collections were used, except for Vinc and Ena analyses for which a dsRNA and a mutant allele were studied (see Supplementary Table 2). The Act $>y+>$ Gal4 flip-out cassette (alone or in combination with TubGal $80^{\text {ts }}$ ) was used either to express the fluorescently tagged gene or to express both the relevant dsRNA and Caax:tBFP that marks the clones. 
Table S2. Alleles and transgenes used in this study.

\begin{tabular}{|c|c|}
\hline Drosophila stock & Reference or Source \\
\hline mirr-Gal4 & (123), BDSC (\#29650) \\
\hline pnr-Gal4 & (97), BDSC (\#3039) \\
\hline HsFlp, Ubi-nls:mRFP, FRT19 & BDSC (\#31418) \\
\hline Tub-Gal80 ${ }^{\text {ts }}$ & BDSC (\#7017) \\
\hline Ubi-nls:GFP & BDSC (\#5629) \\
\hline Ban-nls:GFP & $(23)$ \\
\hline Ubi-PH:GFP & $(124)$ \\
\hline Act $>$ CD2 $>$ Gal4 & BDSC (\#3953) \\
\hline Act $>y+>$ Gal4 & BDSC (\#4780) \\
\hline UAS-Caax:tBFP (Chr III) & (125), made using BDSC (\#55090) \\
\hline UAS-Caax:tBFP (Chr II) & (125), made using BDSC (\#55082) \\
\hline Jub:GFP & (47), BDSC (\#56806) \\
\hline Wts:GFP & (18), BDSC (\#56808) \\
\hline Zasp52:GFP & BDSC (\#58790) \\
\hline UAS-Act5c:GFP & BDSC (\#9258) \\
\hline UAS-zip $^{\mathrm{DN}}:$ YFP & $(49)$ \\
\hline UAS-trbl & $(126)$ \\
\hline UAS-zyx:ChFP & BDSC (\#28875) \\
\hline UAS-cdc2 ${ }^{\text {dsRNA }}$ & BDSC (\#28368) \\
\hline UAS-aurB ${ }^{\text {dsRNA }}$ & BDSC (\#28691) \\
\hline UAS-w ${ }^{\text {dsRNA }}$ & BDSC (\#33644) \\
\hline UAS-actn ${ }^{\text {dsRNA }}$ & BDSC (\#34874) \\
\hline UAS-svn ${ }^{\text {dsRNA }}$ & VDRC (\#43112) \\
\hline UAS-zasp52 ${ }^{\text {dsRNA }}$ & VDRC (\#106177 and \#58198) \\
\hline UAS-zyx ${ }^{\text {dsRNA }}$ & BDSC (\#29591 and BDSC (\#36716) \\
\hline UAS-kst $^{\text {dsRNA }}$ & VDRC (\#37075) and BDSC (\#33933) \\
\hline UAS-cher ${ }^{\text {dsRNA }}$ & VDRC (\#107451) and BDSC (\#26307) \\
\hline UAS-mys ${ }^{\text {dsRNA }}$ & VDRC (\#103704) and BDSC (\#33642) \\
\hline UAS-pax ${ }^{\text {dsRNA }}$ & VDRC (\#107789) and BDSC (\#28695) \\
\hline UAS-rhea ${ }^{\text {dsRNA }}$ & BDSC (\#40399, \#32999 and \#33913) \\
\hline UAS-ena ${ }^{\text {dsRNA }}$ & VDRC (\#106484) \\
\hline UAS-mbs ${ }^{\text {dsRNA }}$ & BDSC (\#32516) \\
\hline FRTG13 $e^{n} a^{210}$ & BDSC (\#25404) \\
\hline UAS-vinc ${ }^{\text {dsRNA }}$ & VDRC (\#105956) \\
\hline $\operatorname{vin} c^{\Delta 3}$ & $(9)$ \\
\hline UAS-LifeAct:GFP & BDSC (\#35544) \\
\hline UAS-GFP:scra & BDSC (\#51348 and \#51349) \\
\hline UAS-Arp3:GFP & BDSC (\#39722 and \#39723) \\
\hline Dys:GFP & BDSC (\#59782) \\
\hline
\end{tabular}




\begin{tabular}{|c|c|}
\hline Fascin:GFP & $(127)$ \\
\hline UAS-Dia:GFP & BDSC (\#56751) \\
\hline Fim:GFP & BDSC (\#59838 and \#51562) \\
\hline p130CAS:GFP & BDSC (\#60508) \\
\hline Zasp66:GFP & BDSC (\#6824) \\
\hline Sep2:GFP & BDSC (\#26257) \\
\hline UAS-Sep4:GFP & BDSC (\#51345) \\
\hline Tm1:GFP & BDSC (\#51537) \\
\hline Tm2:GFP & BDSC (\#6829) \\
\hline Ubi-Ena:GFP & BDSC (\#28798) \\
\hline E-Cad:GFP & $(46)$ \\
\hline Mys:GFP & $(128)$ \\
\hline Rhea:GFP & $(128)$ \\
\hline Vinc:GFP & $(128)$ \\
\hline Cher:YFP & Kyoto Stock Center (\#115123) \\
\hline Kst:YFP & Kyoto Stock Center (\#115518) \\
\hline Pax:YFP & Kyoto Stock Center (\#115540) \\
\hline Dlg:YFP & $(129)$ \\
\hline$\alpha$-cat:GFP & BDSC (\#59405) \\
\hline E-Cad:3xGFP & $(9)$ \\
\hline E-Cad:3xmkate2 & $(9)$ \\
\hline MyoII:3xGFP & $(9)$ \\
\hline MyoII:3xmKate2 & $(9)$ \\
\hline Actn:GFP & This work \\
\hline 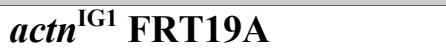 & This work \\
\hline Jub:mkate2 & This work \\
\hline Ubi-Cry2Oligo:ChFP & This work \\
\hline Ubi-Cry2Oligo:ChFP:Jub & This work \\
\hline Wts:CitFP & $(130)$ \\
\hline dIAP1(3.5):GFP & $(55)$ \\
\hline
\end{tabular}




\section{Movie S1: Laser ablation to estimate tissue stress along the $m-l$ and a-p axis during tissue development.}

Time-lapse movie of E-Cad:3xGFP tissue upon laser ablation to estimate mechanical stress along the m-1 axis (bottom) and the a-p axis (top) at $18 \mathrm{hAPF}$ (left) and $26 \mathrm{~h} \mathrm{APF} \mathrm{(right).} \mathrm{Ablation} \mathrm{was} \mathrm{performed} \mathrm{at} \mathrm{t}=0$. Dashed white rectangle outlines the ablated regions. Scale bar: $10 \mu \mathrm{m}$.

\section{Movie S2: Formation of aSFs in the central domain of the tissue.}

A-C: Time-lapse movie of E-Cad:3xmKate2 (A,B) and MyoII:3xGFP (A,C) from 18 hAPF to $26 \mathrm{hAPF}$ in the central posterior region of the tissue, where aSFs form (A). Note that aSFs can sometimes be observed to transiently align between adjacent cells and to form multicellular actomyosin structures along the tissue's main stress axis. White box in A indicates the region, which is shown in the close-ups in B and C. Yellow arrowheads indicate aSFs (C). Scale bars: $20 \mu \mathrm{m}(\mathrm{A}) ; 10 \mu \mathrm{m}(\mathrm{B}, \mathrm{C})$.

\section{Movie S3: aSF laser ablation and cell shape changes.}

Time-lapse movie of E-Cad:x3mKate2 and MyoII:3xGFP localization upon aSF laser ablation. Dashed white box: ablated region. Time was set to $0 \mathrm{~s}$ at the time of ablation. Yellow arrowheads: position of the aSF tips prior and upon ablation. Scale bar: $2 \mu \mathrm{m}$.

\section{Movie S4: AJ laser ablation and cell shape changes.}

Time-lapse movie of E-Cad:x3mKate2 and MyoII:3xGFP localization upon AJ laser ablation. Dashed white box: ablated region. Time was set to 0s at the time of ablation. Yellow arrowheads: positions of the TCJs prior to and after ablation. Scale bar: $2 \mu \mathrm{m}$.

\section{Movie S5: AJ ablation followed by aSF ablation.}

Time-lapse movie of E-Cad:x3mKate2 and MyoII:3xGFP localization upon laser ablation of first the AJ and then the aSF of one cell (around 23s later). Note that no significant aSF reorientation is observed and that the AJ does not heal prior to aSF ablation. AJ time (orange) was set to 0 s at the time of AJ ablation, and aSF time (blue) was set to $0 \mathrm{~s}$ at the time of aSF ablation. Orange and blue dashed box: AJ and aSF ablated regions, respectively. Scale bar: $2 \mu \mathrm{m}$.

\section{Movie S6: aSF ablation followed by AJ ablation.}

Time-lapse movie of E-Cad:x3mKate2 and MyoII:3xGFP localization upon laser ablation first of the aSF and then of the AJ of one cell (around 30s later). Note that no significant AJ reorientation is observed and that no aSF healing process was observed in any of the aSF ablations performed. aSF time (blue) was set to $0 \mathrm{~s}$ at the time of aSF ablation, and AJ time (orange) was set to $0 \mathrm{~s}$ at the time of AJ ablation. Orange and blue dashed box: AJ and aSF ablated regions, respectively. Scale bar: $2 \mu \mathrm{m}$.

\section{Movie S7: Multiple aSF ablations in a single cell.}

Time-lapse movie of E-Cad:x3mKate2 and MyoII:3xGFP localization upon laser ablation of four aSFs within an individual cell. Dashed white box: ablated regions. Time was set to $0 \mathrm{~s}$ at the time of ablation. Scale bar: $2 \mu \mathrm{m}$.

Movie S8: Optogenetic clustering of Jub and Wts.

(A,B) Time-lapse movie of Wts:GFP (A,B), Cry2Olig:mChFP (A) or Cry2Olig:mChFP:Jub (B) dynamics upon $492 \mathrm{~nm}$ light-illumination. Scale bar: $2 \mu \mathrm{m}$.

Movie S9: Jub and Wts junctional dynamics during aSF movement.

(A-B') Time-lapse movie of MyoII:3xGFP (A) and Jub:mKate2 (green, A and fire, A') as well as MyoII:3xmKate2 (B) and Wts:CitFP (green, B and fire, B') localizations during aSF displacement. Yellow arrowheads: Jub:mKate 2 or Wts:CitFP clusters. Scale bar: $2 \mu \mathrm{m}$. 
Movie S10: Jub and Wts junctional dynamics during aSF ablation.

(A-B') Time-lapse movie of MyoII:mKate2 (A) and Jub:GFP (green, A and fire, A') as well as MyoII:3xmKate2 (B) and Wts:CitFP (green, B and fire, B') localizations upon aSF ablation. Dashed white box: ablated region. Yellow arrowheads: Jub:GFP or Wts:CitFP clusters. Scale bar: $2 \mu \mathrm{m}$.

Movie S11: aSF nucleation, peeling and breaking.

Time-lapse movie of MyoII:3xGFP and E-Cad:3xmKate2 during aSF nucleation at a TCJ $(\mathrm{t}=0)$, peeling and breaking at another TCJ ( $\mathrm{t}=42 \mathrm{~min})$. Time was set to $0 \mathrm{~min}$ at the time of aSF nucleation (red arrowhead). Yellow arrowheads indicate aSF tips. Note that the aSF breaks at the TCJ. Scale bar: $2 \mu \mathrm{m}$. 

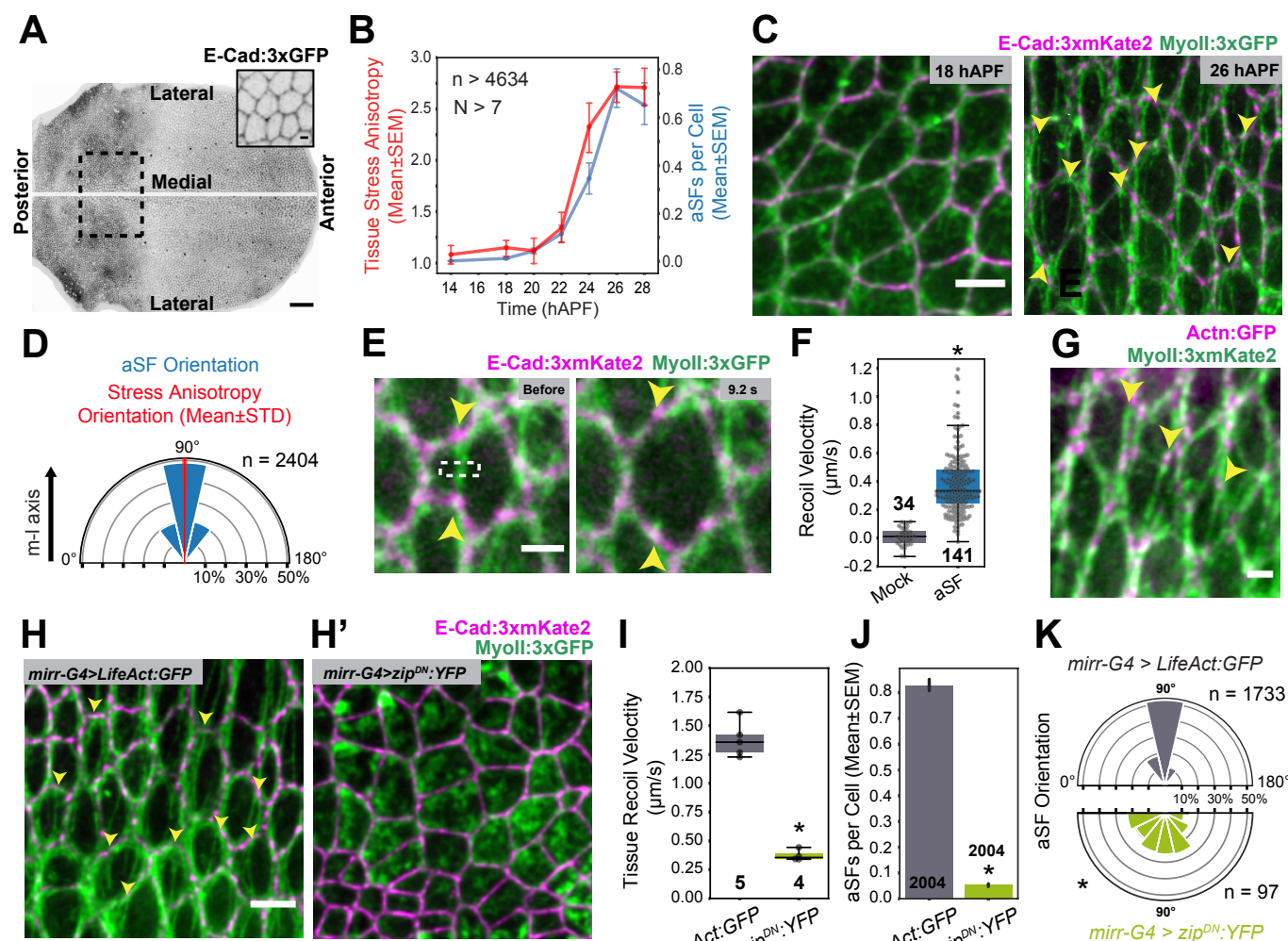

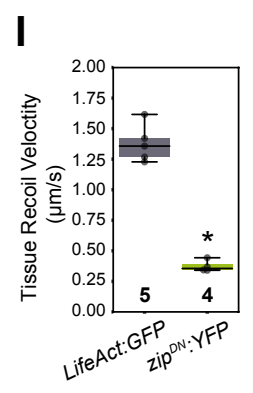

M

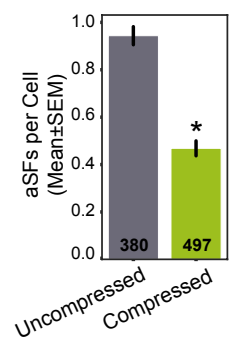

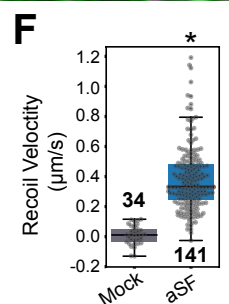

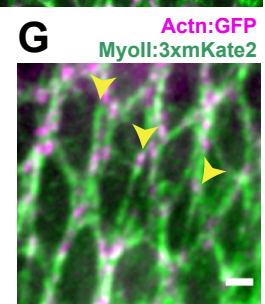

K

$\mathbf{J} \quad \mathbf{K}$

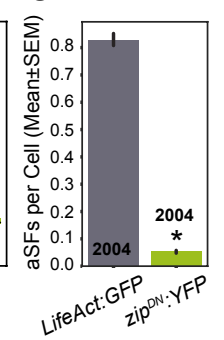

N
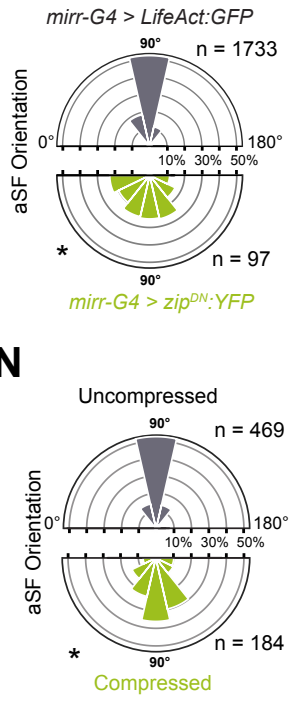

Figure 1

Lopez-Gay et al. 

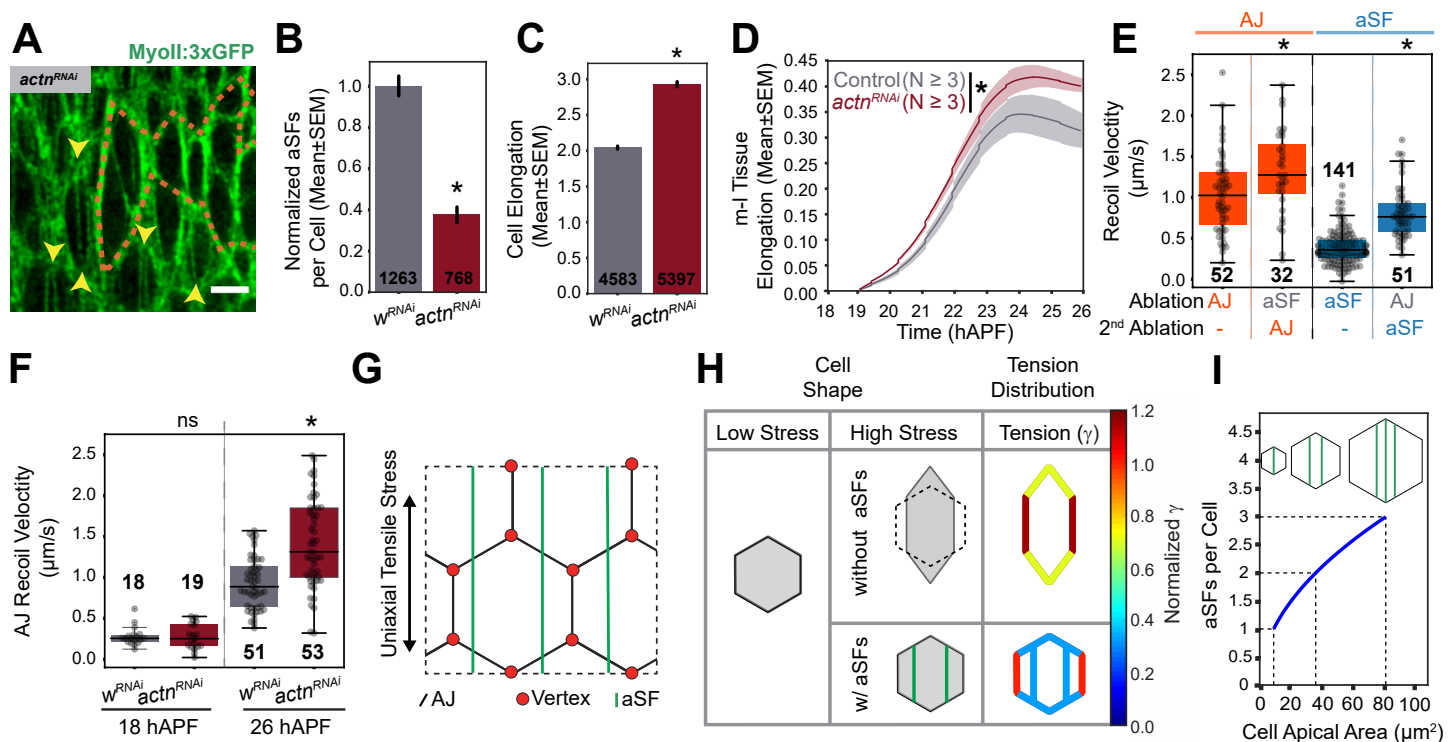

G
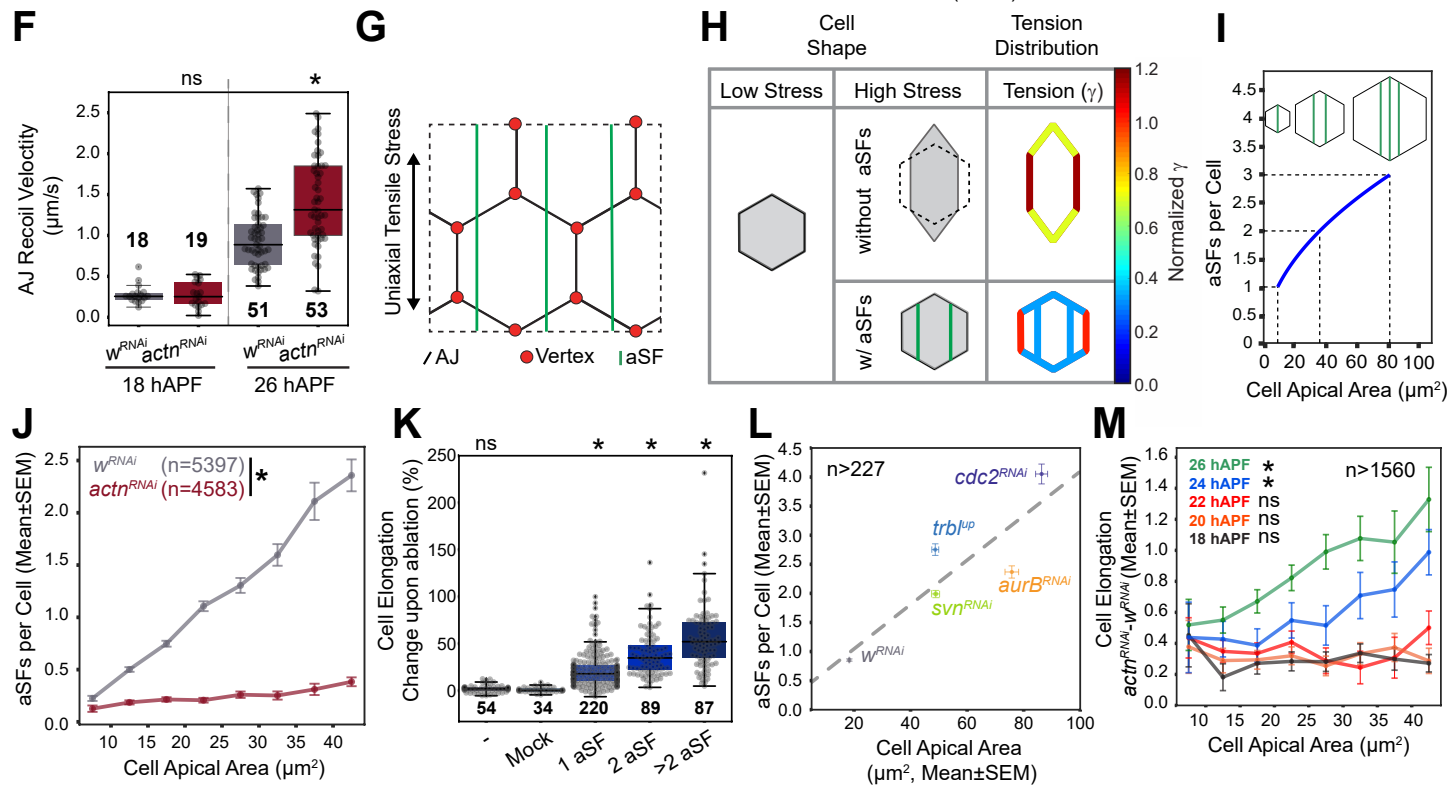

M

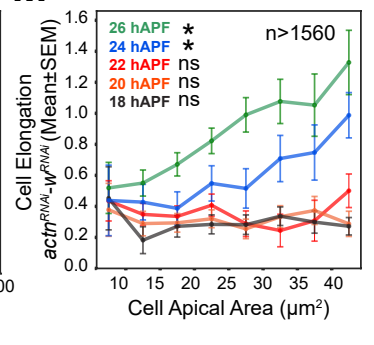

Figure 2

Lopez-Gay et al. 

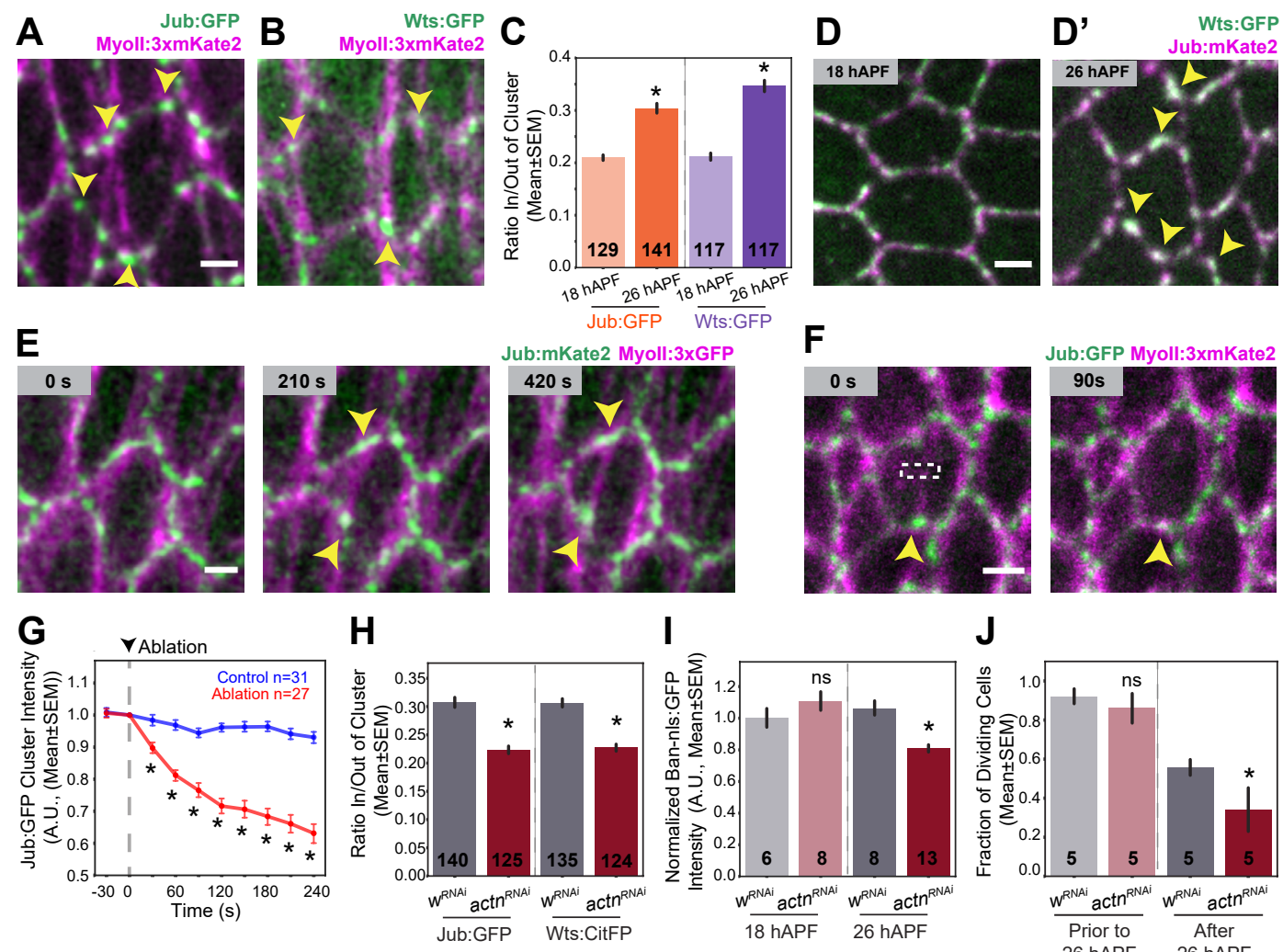

J

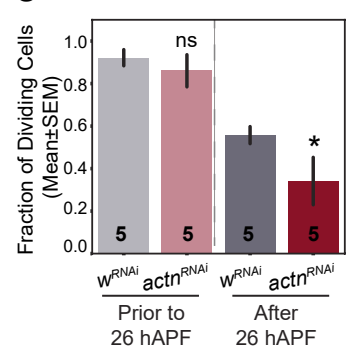

Figure 3

Lopez-Gay et al. 

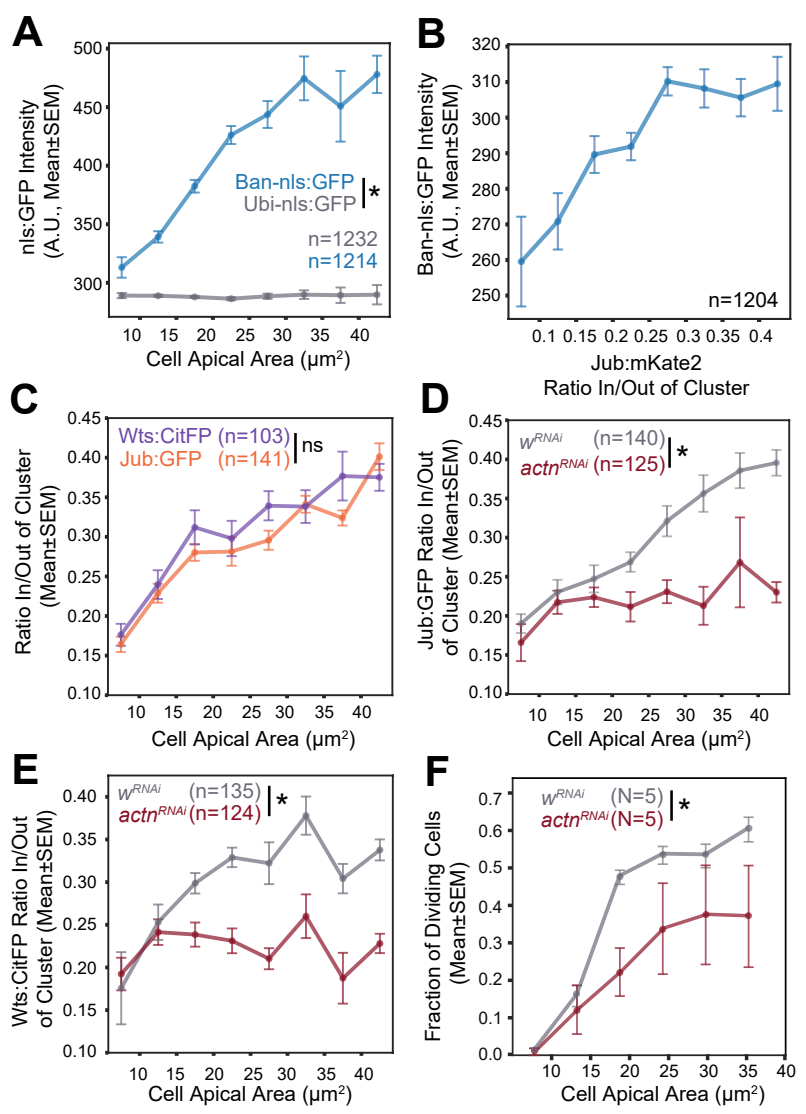

Figure 4

Lopez-Gay et al. 
A

E-Cad:3xmKate2 Myoll:3xGFP
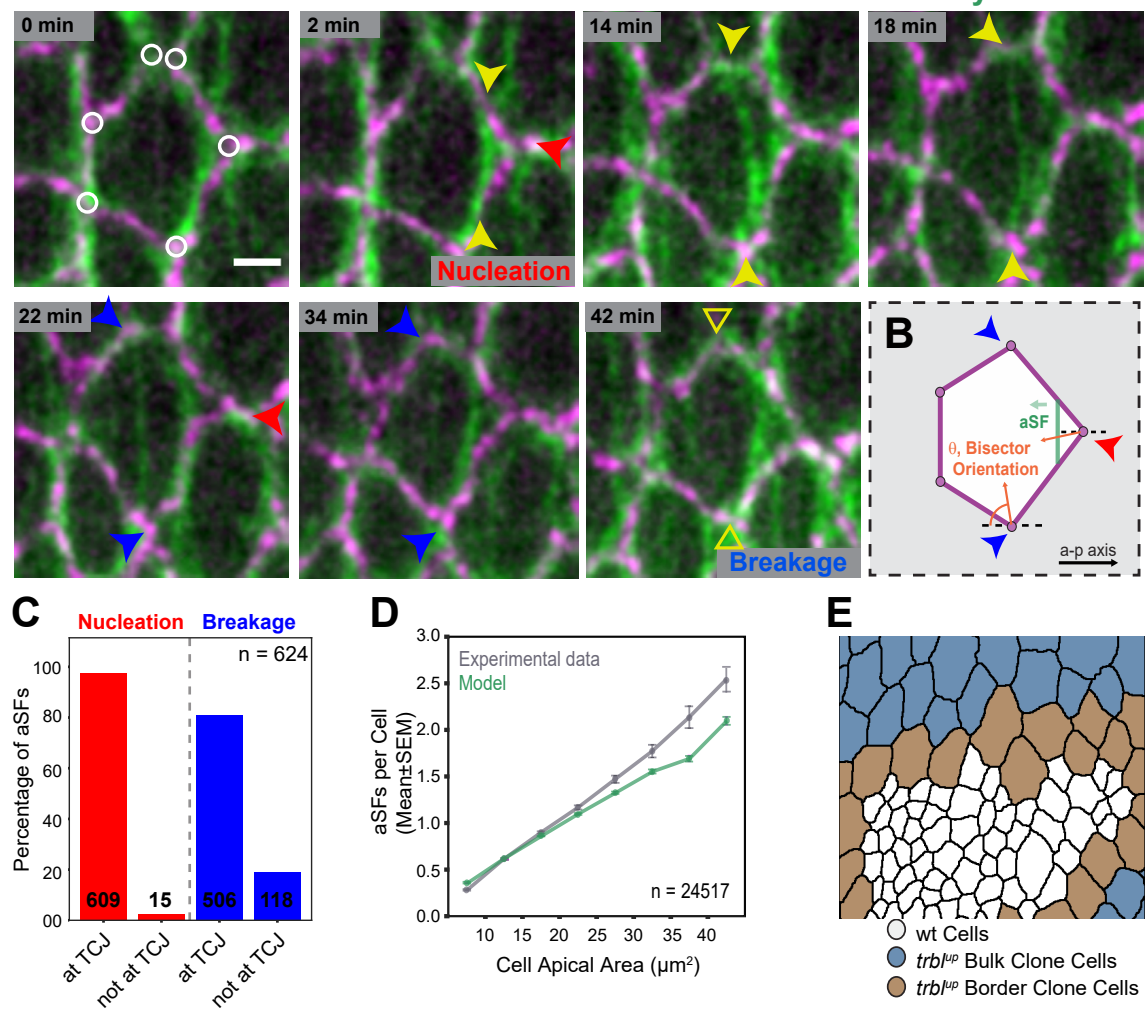

D

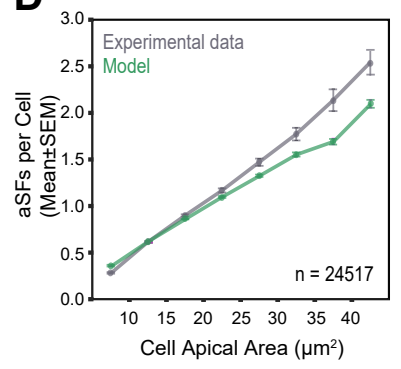

E

$\mathbf{F}$

G

H
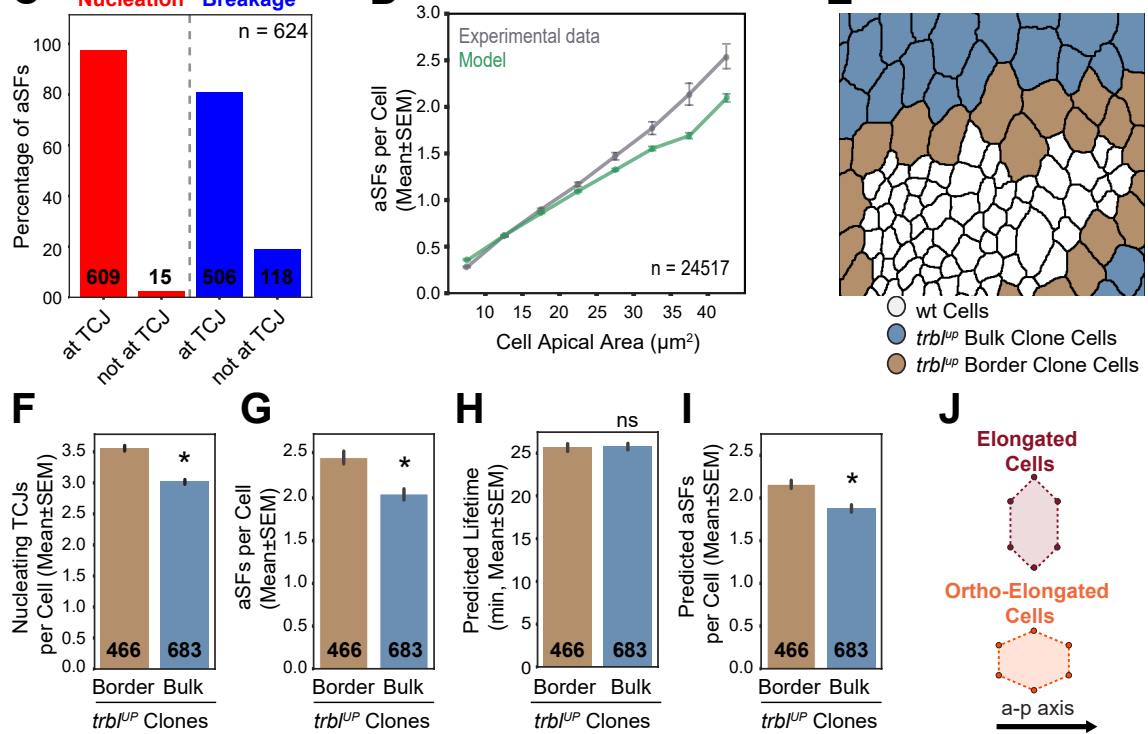

I

trb/ ${ }^{\mu p}$ Border Clone Cells

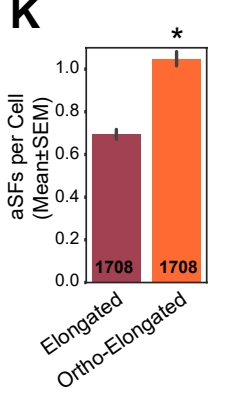

$\mathbf{L}$
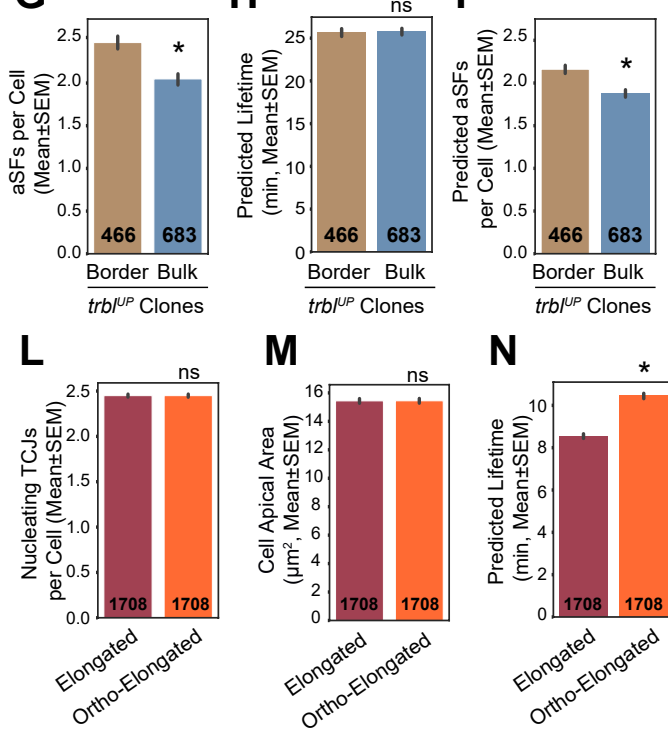

J

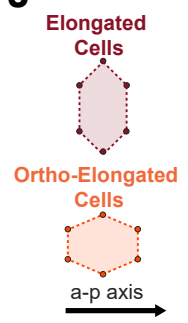

$\mathbf{N}$

0
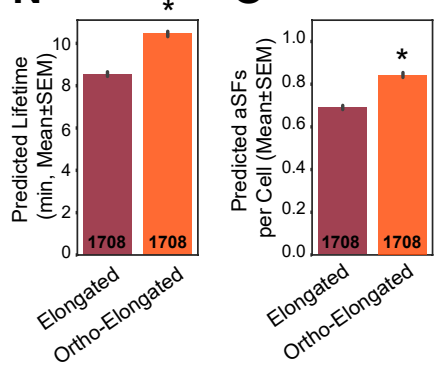

Figure 5

Lopez-Gay et al. 

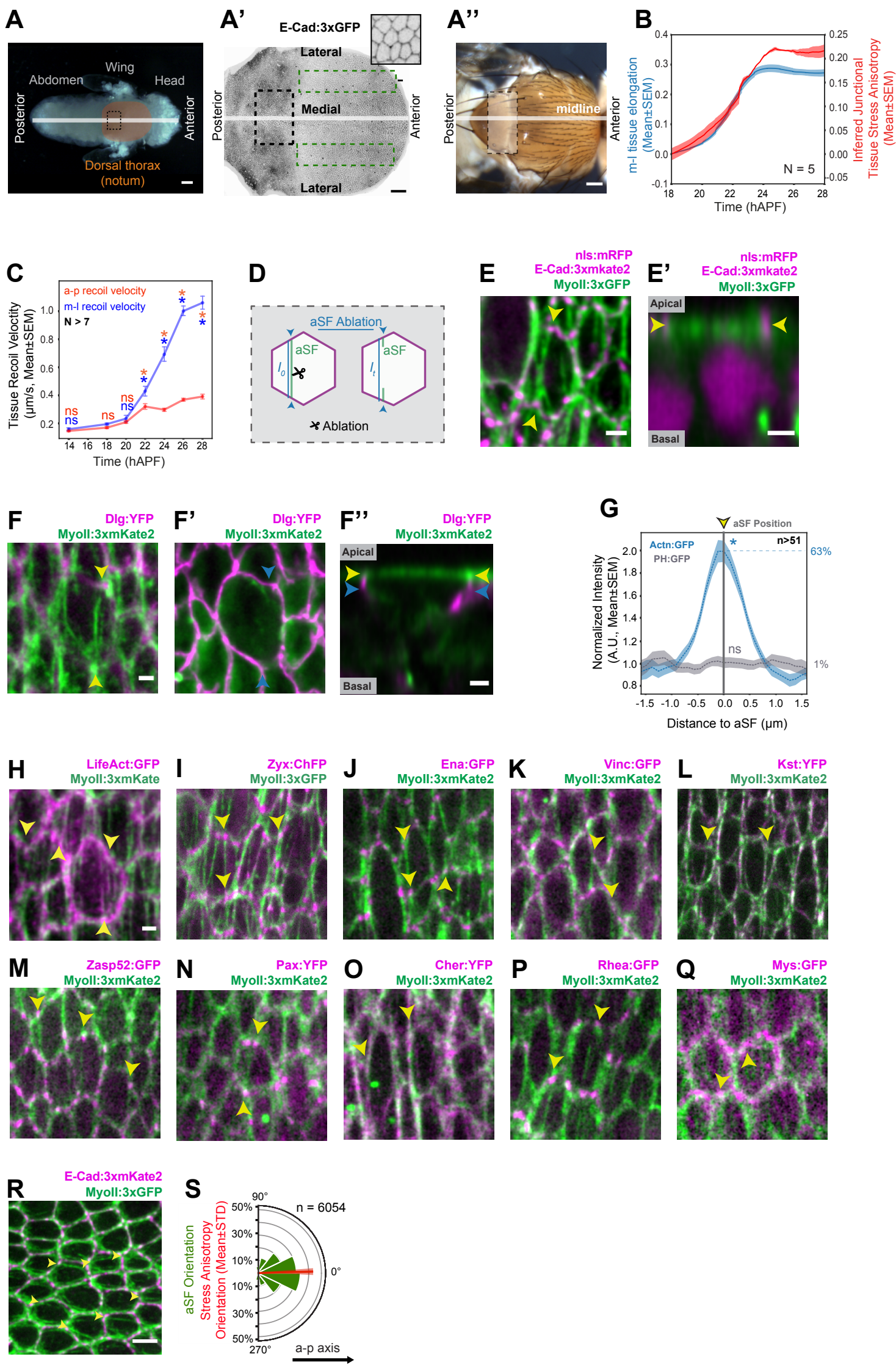

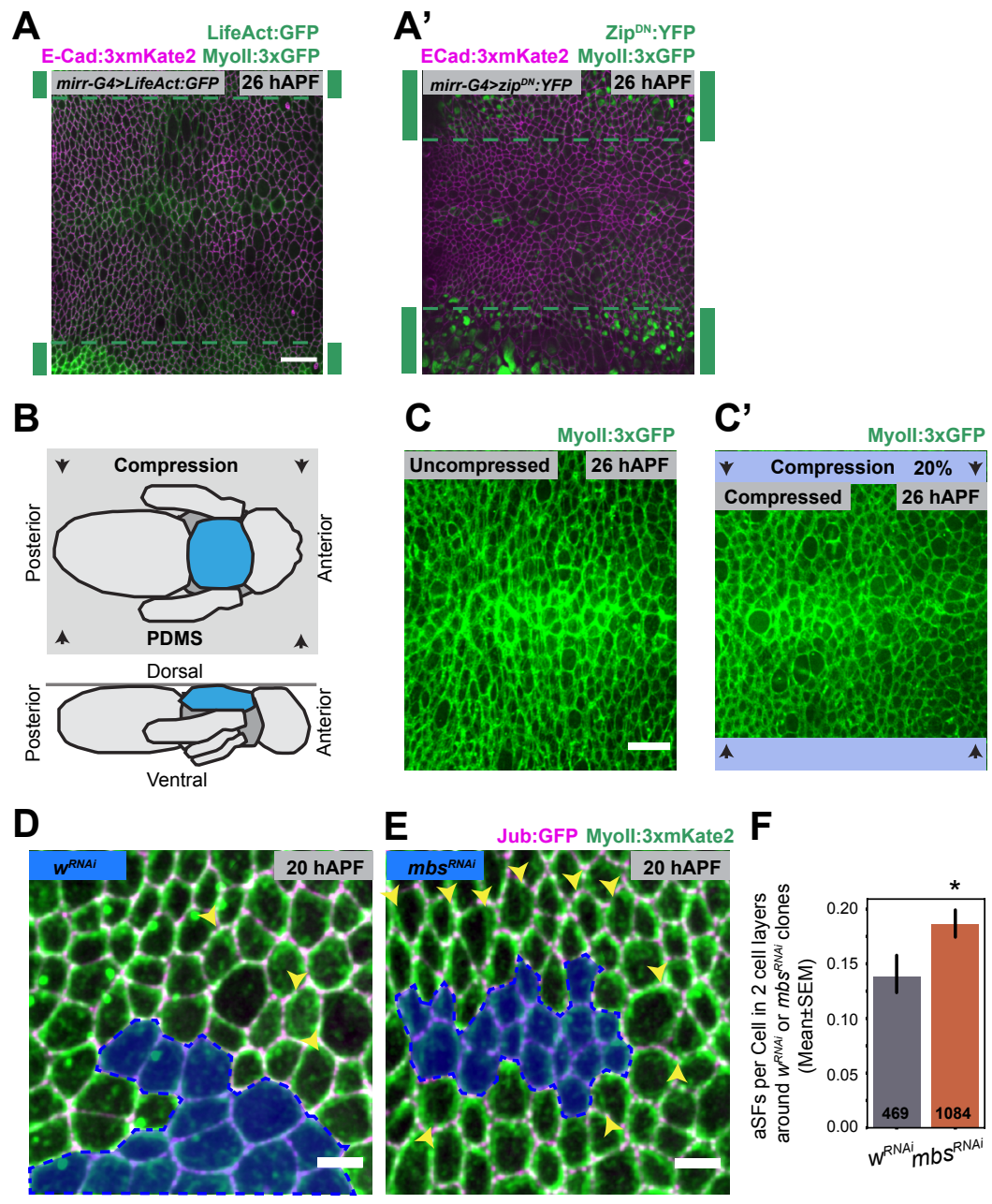

E

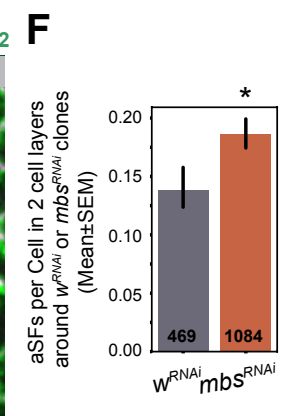

Supplementary Figure 2
Lopez-Gay et al. 

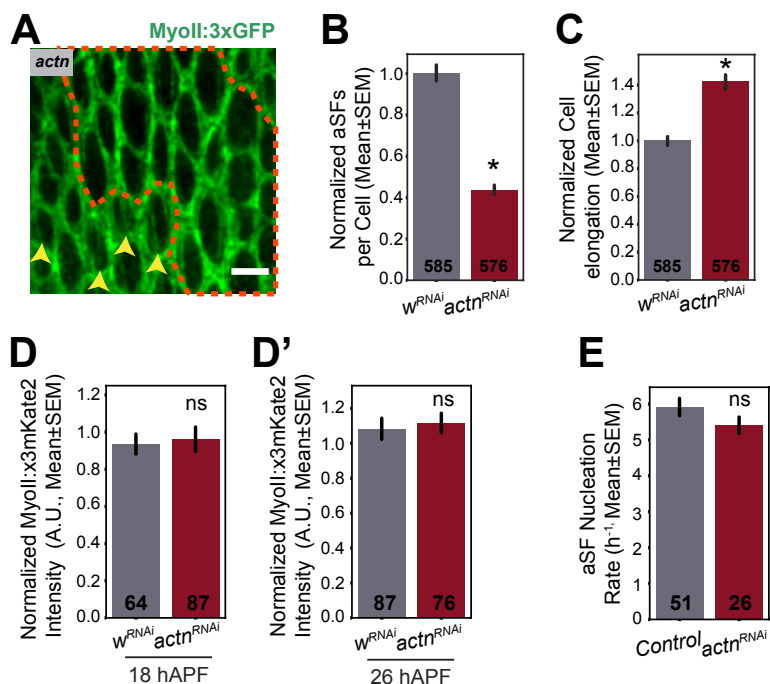

E

$E^{\prime}$
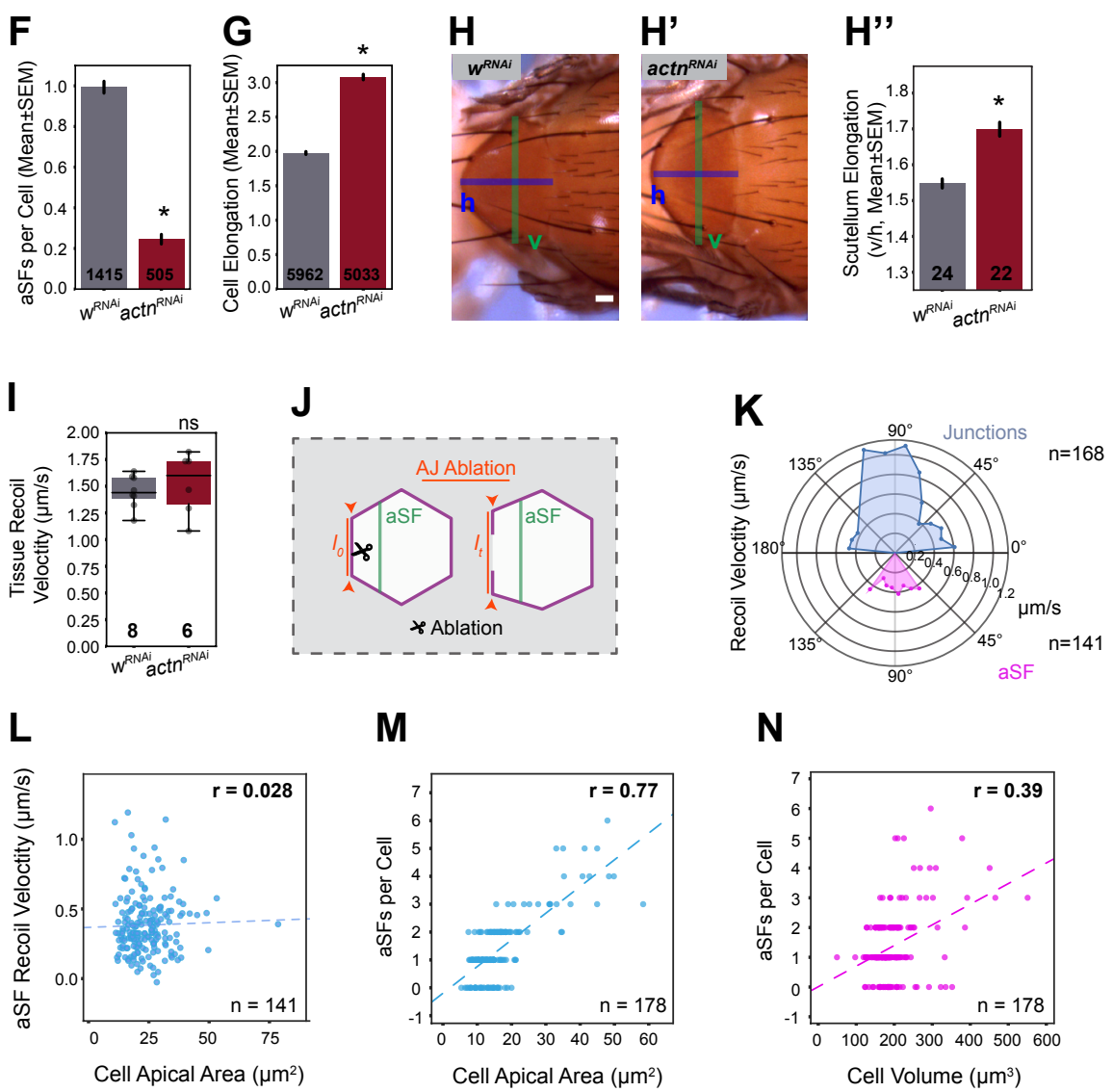

M

\section{$\mathbf{N}$}
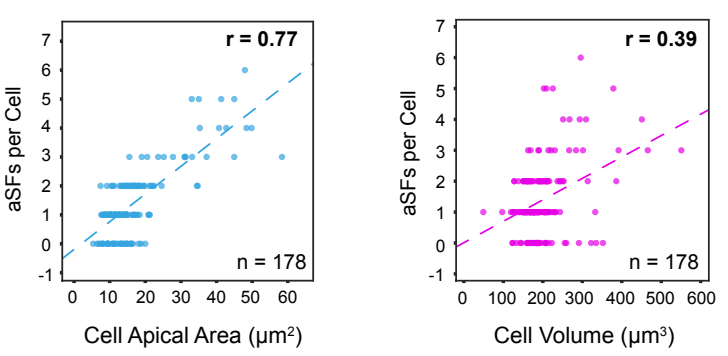

0

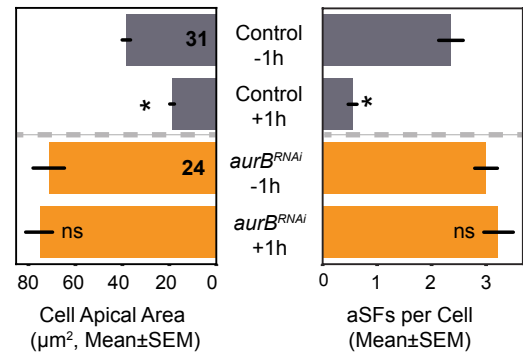

$\mathbf{P}$

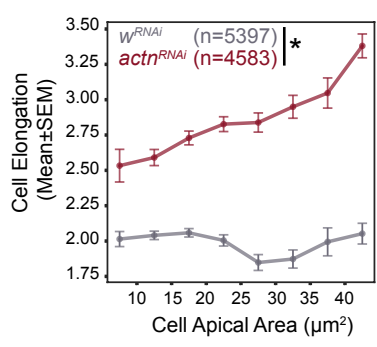

Q

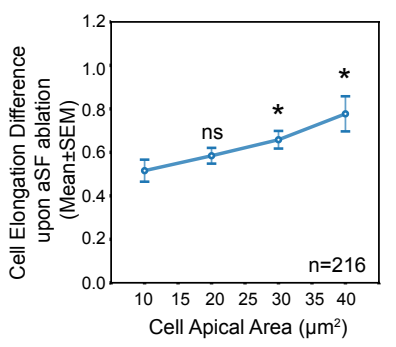



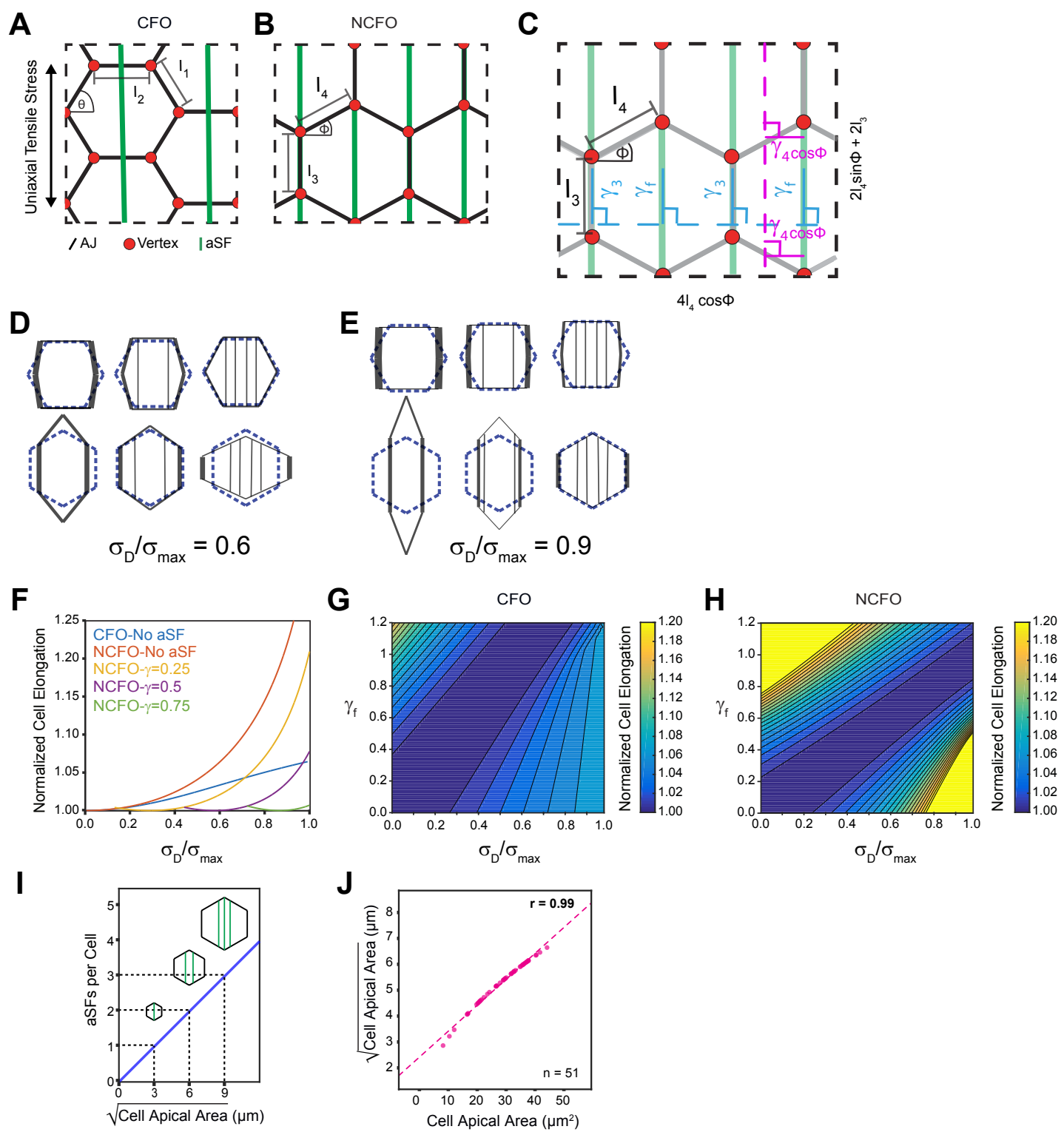

Supplementary Figure 4 Lopez-Gay et al. 

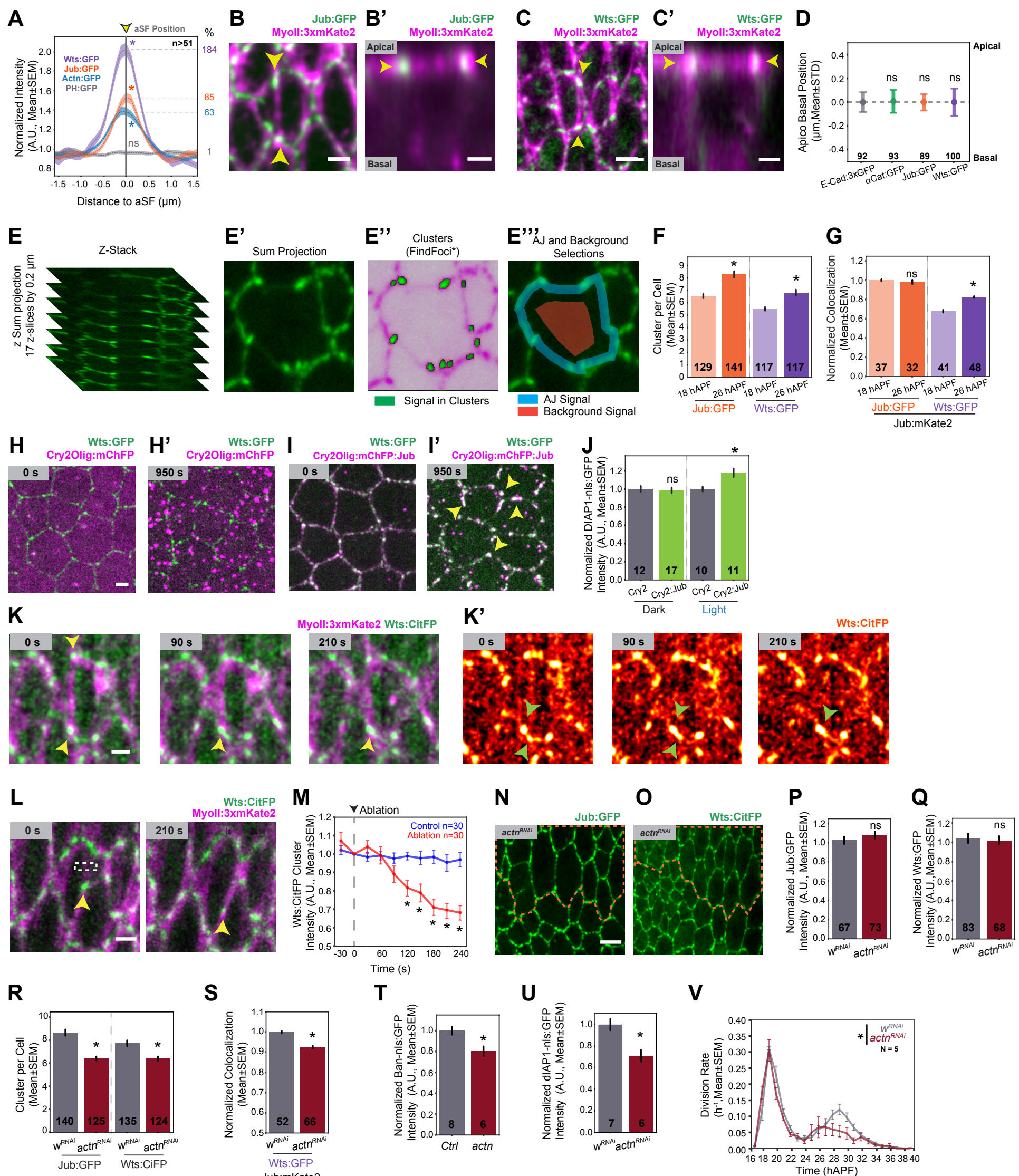

S

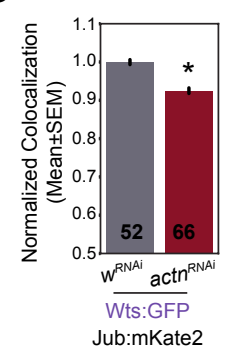

$\mathbf{T}$

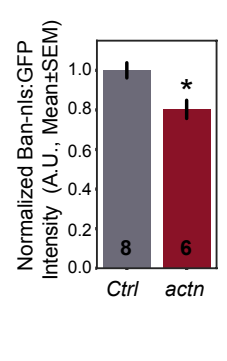

U

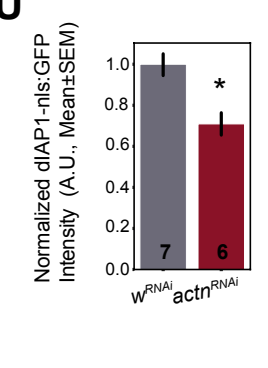

V

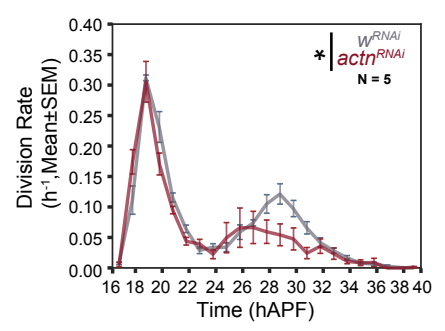

Supplementary Figure 5 Lopez-Gay et al. 
A

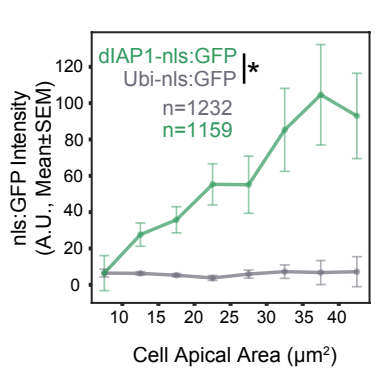

\section{D}

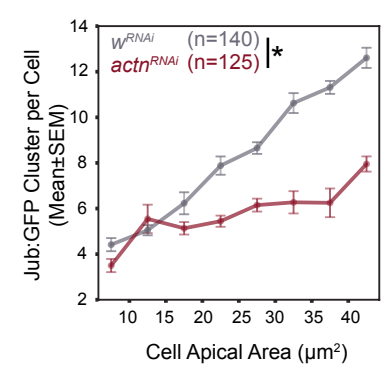

B

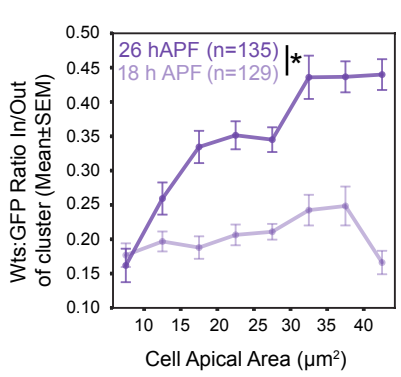

E

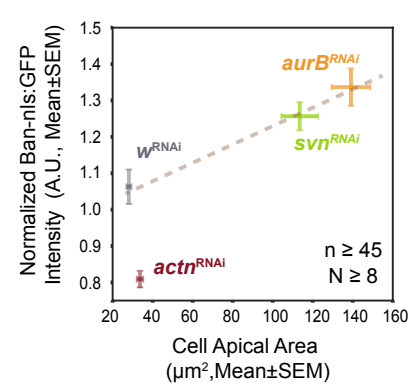

C

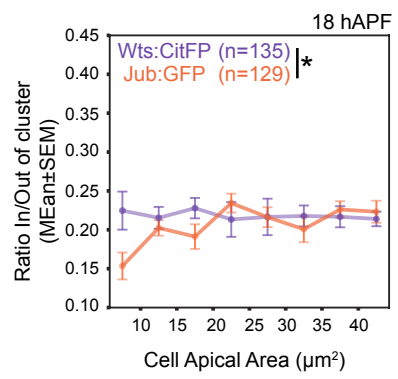

$\mathbf{F}$

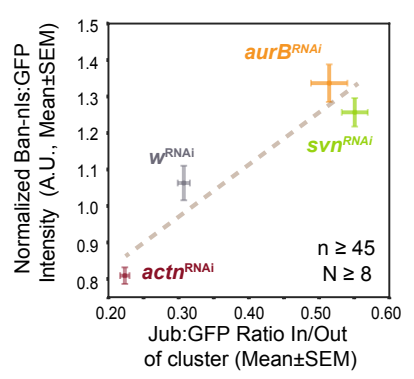

Supplementary Figure 6 Lopez-Gay et al. 

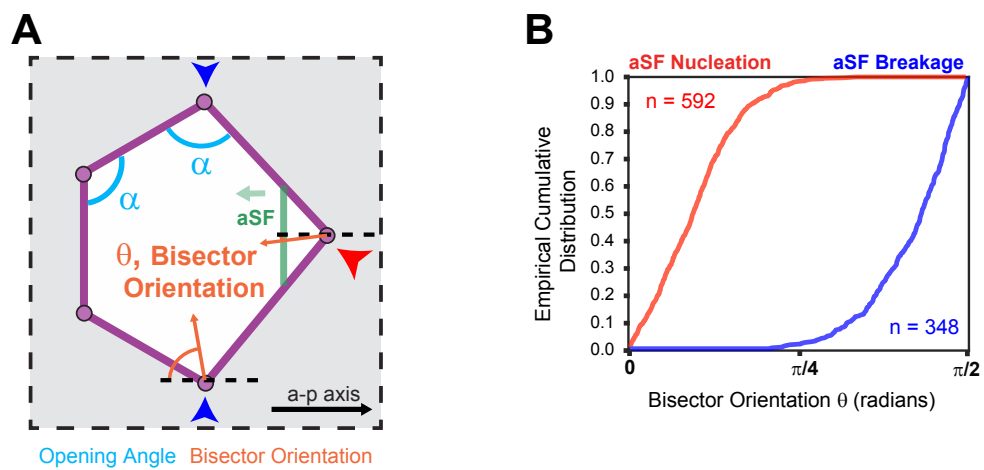

C

D

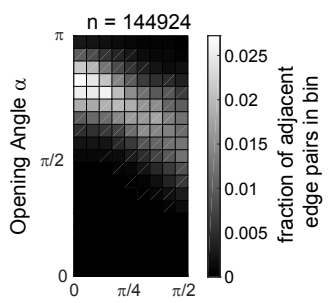

Bisector Orientation $\theta$

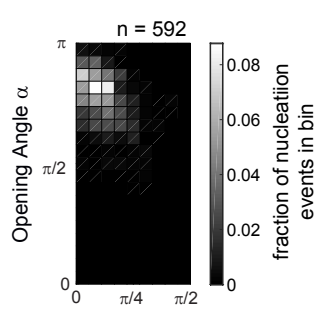

E

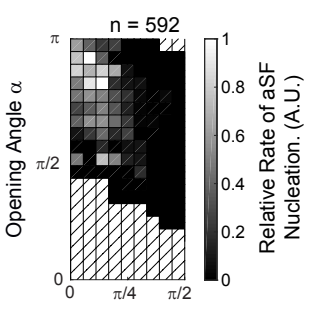

Bisector Orientation $\theta$

Bisector Orientation $\theta$

$\mathbf{F}$

G
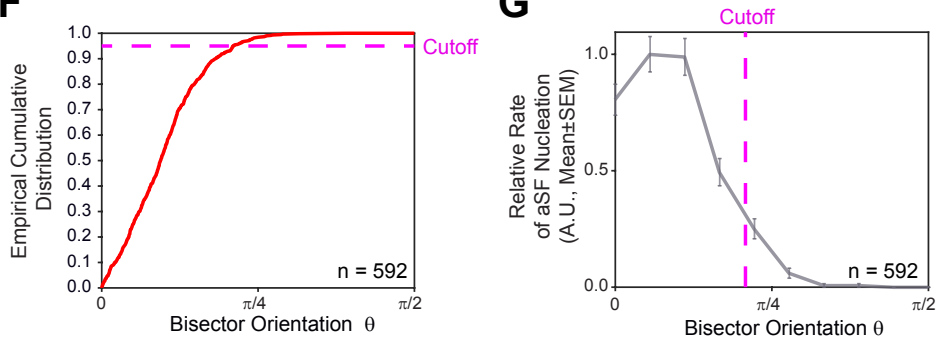

H

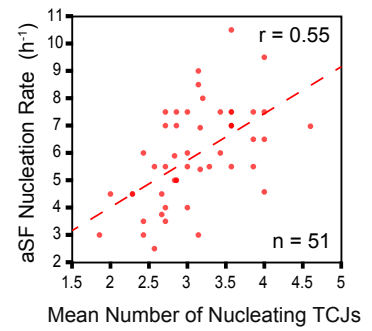

I
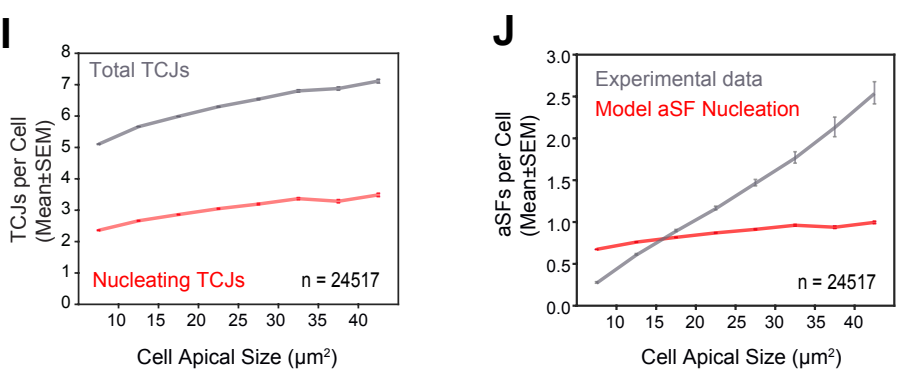

Supplementary Figure 7 Lopez-Gay et al. 
A

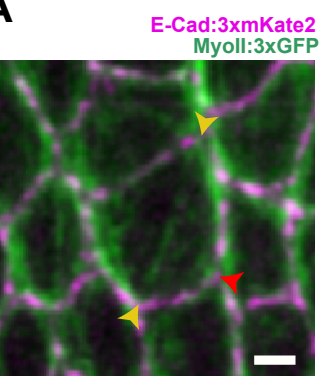

E

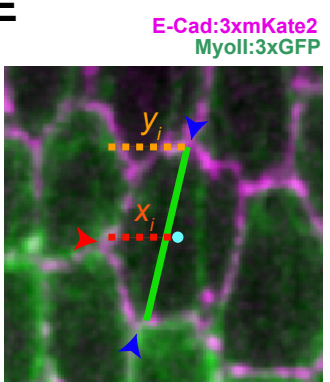

H

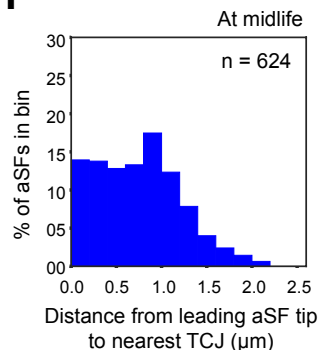

$\mathbf{L}$

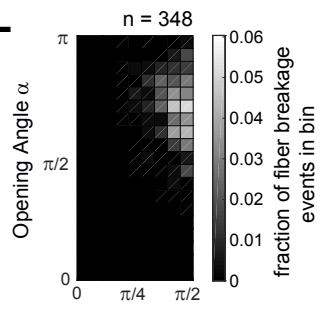

Bisector Orientation $\theta$

$\mathbf{N}$

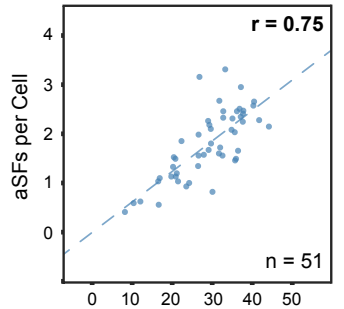

Cell Apical Area $\left(\mu \mathrm{m}^{2}\right)$

S

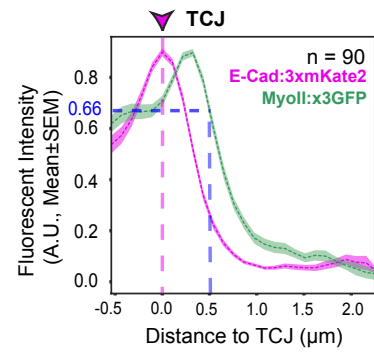

B

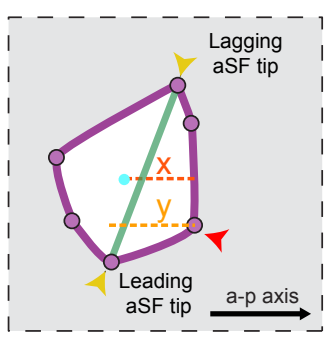

$\mathbf{F}$
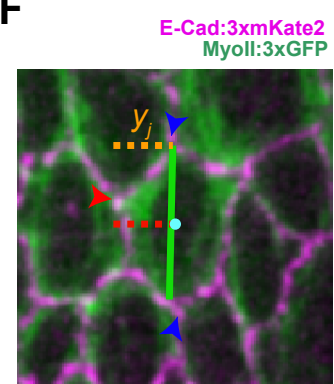

I

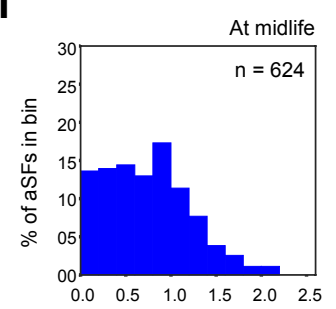

Distance from swapped leading aSF tip to nearest TCJ $(\mu \mathrm{m})$

M

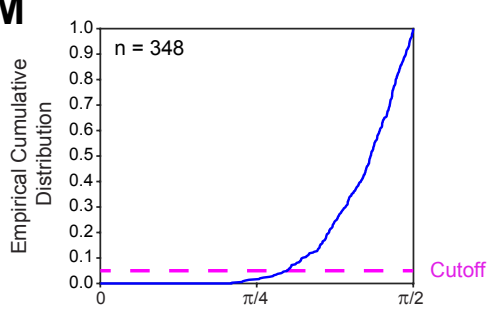

Bisector Orientation $\theta$

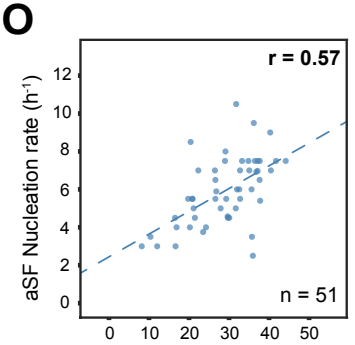

Cell Apical Area $\left(\mu \mathrm{m}^{2}\right)$

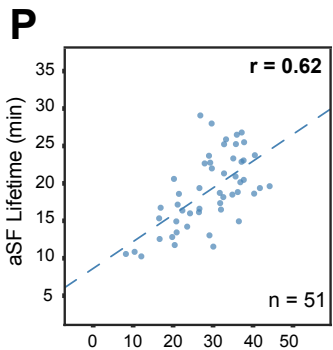

Cell Apical Area $\left(\mu \mathrm{m}^{2}\right)$
Q

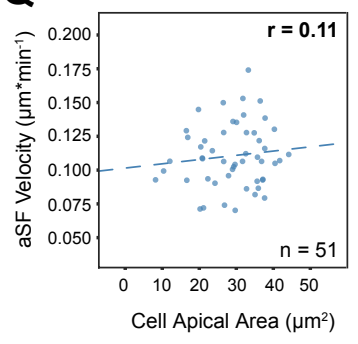

K

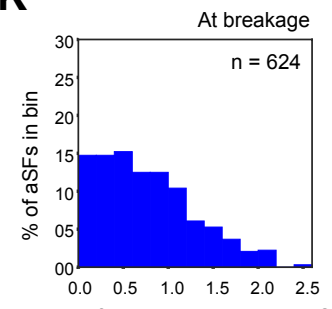

Distance from swapped leading aSF tip to nearest TCJ $(\mu \mathrm{m})$

$\mathbf{T}$

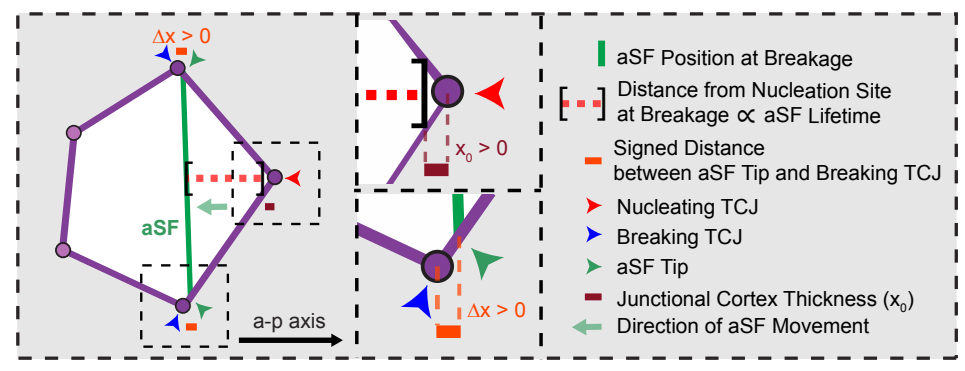

$\mathbf{R}$

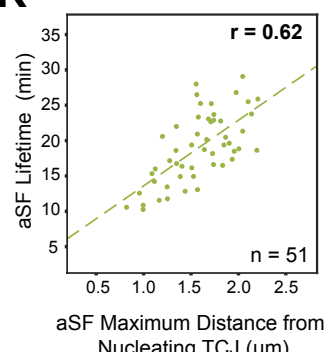




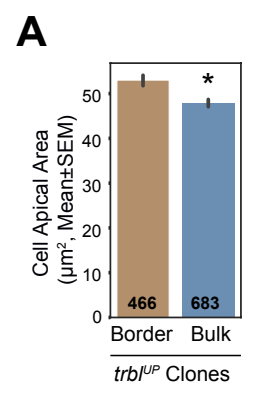

B
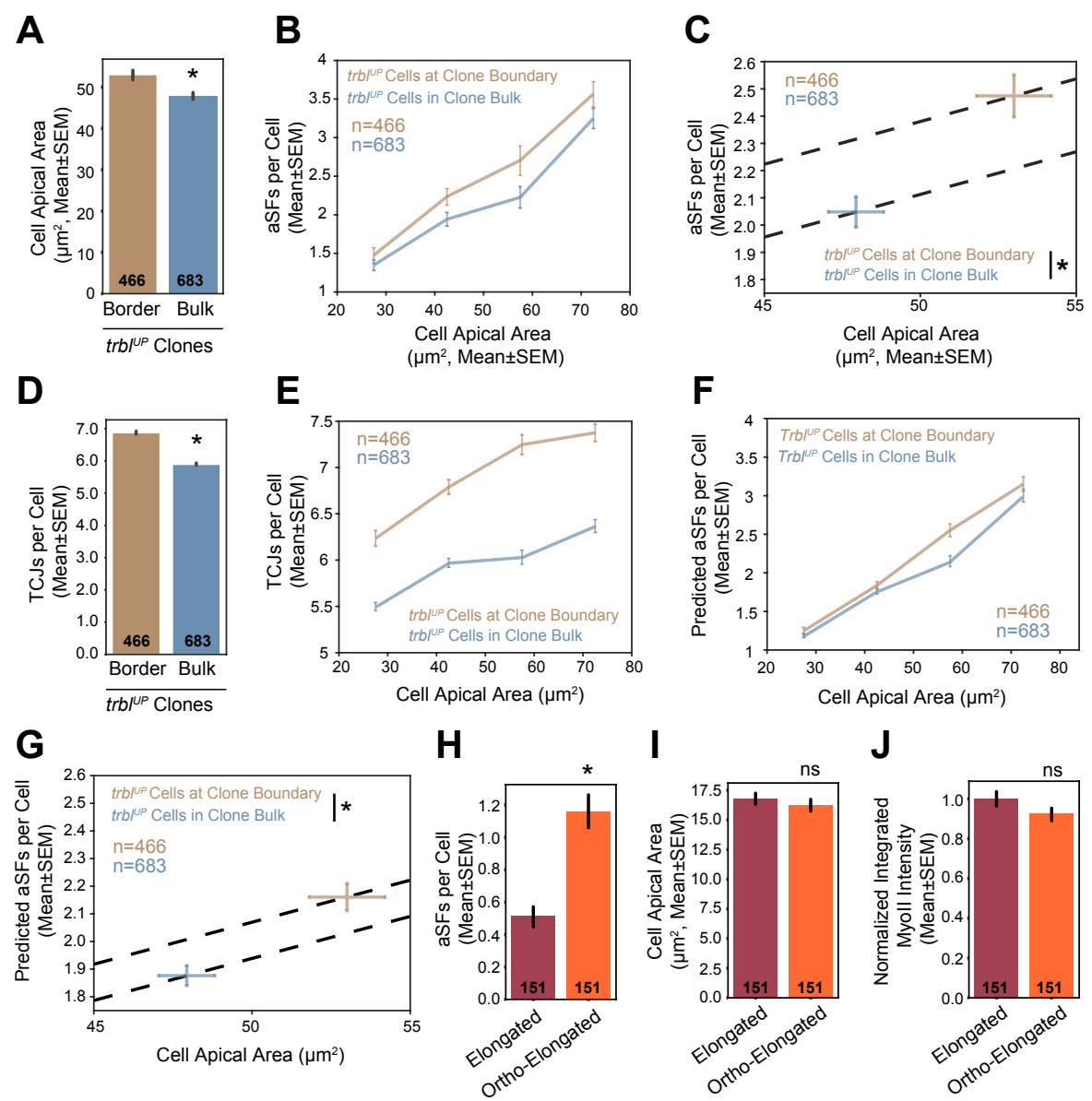

\section{Supplementary Figure 9 Lopez-Gay et al.}




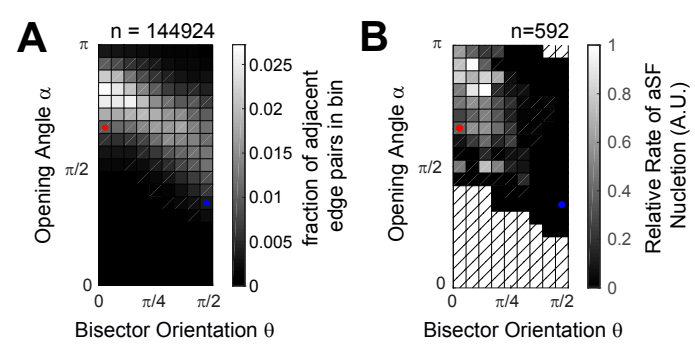

C

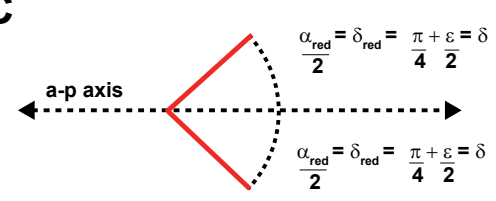

D

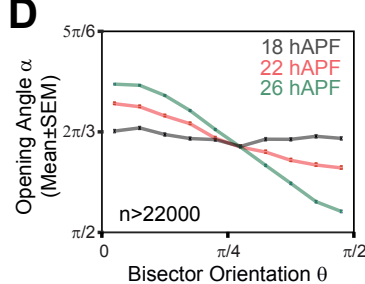

C'

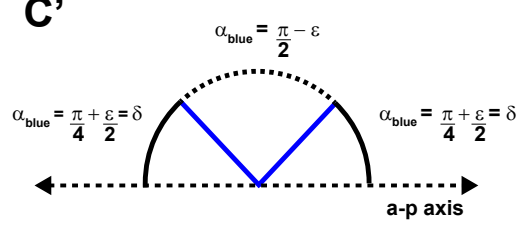

Supplementary Figure 10

Lopez-Gay et al. 